\title{
LARS HOLMBERG
}

HUMAN IN COMMAND MACHINE LEARNING

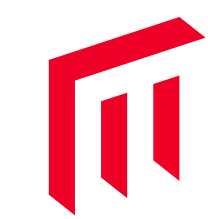





$$
-
$$


Malmö University,

Studies in Computer Science No 16, Licentiate Thesis

(c) Lars Holmberg, 2021

Illustration cover and pg. 1 by Anna Hasslöf

ISBN 978-91-7877-186-8 (print)

ISBN 978-91-7877-187-5 (pdf)

DOI 10.24834/isbn.9789178771875

Printed by Holmbergs, Malmö 2021 


\section{LARS HOLMBERG}

HUMAN IN COMMAND MACHINE LEARNING

Malmö University, 2021

Faculty of Technology and Society Department of Media and Computer Science 


\section{Studies in Computer Science}

Faculty of Technology and Society

Malmö University

1. Jevinger, Åse. Toward intelligent goods: characteristics, architectures and applications, 2014, Doctoral dissertation.

2. Dahlskog, Steve. Patterns and procedural content generation in digital games: automatic level generation for digital games using game design patterns, 2016, Doctoral dissertation.

3. Fabijan, Aleksander. Developing the right features: the role and impact of customer and product data in software product development, 2016, Licentiate thesis.

4. Paraschakis, Dimitris. Algorithmic and ethical aspects of recommender systems in e-commerce, 2018, Licentiate thesis.

5. Hajinasab, Banafsheh. A Dynamic Approach to Multi Agent Based Simulation in Urban Transportation Planning, 2018, Doctoral dissertation.

6. Fabijan, Aleksander. Data-Driven Software Development at Large Scale, 2018, Doctoral dissertation.

7. Bugeja, Joseph. Smart Connected Homes: Concepts, Risks, and Challenges, 2018, Licentiate thesis.

8. Alkhabbas, Fahed. Towards Emergent Configurations in the Internet of Things, 2018, Licentiate thesis.

9. Paraschakis, Dimitris. Sociotechnical Aspects of Automated Recommendations: Algorithms, Ethics, and Evaluation, 2020, Doctoral dissertation.

10. Tegen, Agnes. Approaches to Interactive Online Machine Learning, 2020, Licentiate thesis.

11. Alvarez, Alberto. Exploring the Dynamic Properties of Interaction in Mixed-Initiative Procedural Content Generation, 2020, Licentiate thesis.

12. Alkhabbas, Fahed. Realizing Emergent Configurations in the Internet of Things, 2020, Doctoral dissertation.

13. Ashouri, Majid. Towards Supporting IoT System Designers in Edge Computing Deployment Decisions, 2021, Licentiate thesis.

14. Bugeja, Joseph. On Privacy and Security in Smart Connected Homes, 2021, Doctoral dissertation.

15. Azadvar, Ahmad. Predictive Psychological Player Profiling, 2021, Licentiate thesis.

16. Holmberg, Lars. Human In Command Machine Learning, 2021, Licentiate thesis. 
Karin, Göran 



\section{ABSTRACT}

Machine Learning (ML) and Artificial Intelligence (AI) impact many aspects of human life, from recommending a significant other to assist the search for extraterrestrial life. The area develops rapidly and exiting unexplored design spaces are constantly laid bare. The focus in this work is one of these areas; ML systems where decisions concerning ML model training, usage and selection of target domain lay in the hands of domain experts.

This work is then on ML systems that function as a tool that augments and/or enhance human capabilities. The approach presented is denoted Human In Command ML (HIC-ML) systems. To enquire into this research domain design experiments of varying fidelity were used. Two of these experiments focus on augmenting human capabilities and targets the domains commuting and sorting batteries. One experiment focuses on enhancing human capabilities by identifying similar hand-painted plates. The experiments are used as illustrative examples to explore settings where domain experts potentially can: independently train an ML model and in an iterative fashion, interact with it and interpret and understand its decisions.

HIC-ML should be seen as a governance principle that focuses on adding value and meaning to users. In this work, concrete application areas are presented and discussed. To open up for designing ML-based products for the area an abstract model for HIC-ML is constructed and design guidelines are proposed. In addition, terminology and abstractions useful when designing for explicability are presented by imposing structure and rigidity derived from scientific explanations. Together, this opens up for a contextual shift in ML and makes new application areas probable, areas that naturally couples the usage of AI technology to human virtues and potentially, as a consequence, can result in a democratisation of the usage and knowledge concerning this powerful technology.

Keywords: Human-centred AI/ML, Explainable AI, Machine Learning, Human In the Loop ML 



\section{SAMMANFATTNING}

Artificiell Intelligens (AI) och framför allt Maskininlärning (ML) påverkar våra liv på många sätt, från att rekommendera en möjlig livspartner till att hjälpa till i sökandet efter utomjordiskt liv. ML utvecklas kontinuerligt och därmed blottläggs nya outforskade frågeställningar. Focus för denna avhandling är ett av dessa områden; hur kan man utforma ML-system där beslut om systemets träning, användning och användningsområde tas av en domänexpert. ML system som då, i likhet med ett verktyg, förstärker och/eller utvidgar domänexpertens förmågor.

Denna typ av ML-System benämns här ML-system under mänskligt kommando (HIC-ML) och utforskas genom tre prototypbaserade designexperiment. Två av experimenten fokuserar på att förstärka mänskliga förmågor för de specifika användningsområdena pendling och batterisortering medan det tredje fokuserar på att skapa ett verktyg som utvidgar den mänskliga förmågan att identifiera föremål som liknar varandra, i detta fall, handmålade tallrikar. Experimenten genomförs för att på ett illustrativt och generativt sätt utforska användningsområden där en domänexpert självständigt och iterativt kan träna ett ML-system, interagera med det samt förklara och förstå dess beslut.

HIC-ML ses här som en princip för styrning av inlärningsprocessen med fokus på meningsskapande och mervärde för domänexperter. I avhandlingen diskuteras framtida applikationsområden, detta kompletteras med en abstrakt modell för HIC-ML och förslag på riktlinjer för designarbetet. Terminologier och abstraktioner definieras för HIC-ML system som utvidgar mänskliga kognitiva förmågor. Denna terminologi och dessa abstraktioner baseras på rigiditeten och strukturen hos vetenskapliga förklaringar. Tillsammans troliggör detta ett kontextuellt användarbaserat fokus och nya användningsområden för ML. Områden, som genom sitt fokus på domänexperter, kopplar användningen av AI-teknologi till mänskliga dygder och därmed, som en konsekvens, kan resultera i en demokratisering av användandet och spridandet av kunskaper relaterade till denna kraftfulla teknologi. 



\section{LIST OF PUBLICATIONS}

\section{Included publications}

Holmberg, L., Davidsson, P., \& Linde, P. (2020). Evaluating Interpretability in Machine Teaching. In Highlights in Practical Applications of Agents, Multi-Agent Systems, and Trustworthiness. Communications in Computer and Information Science book series, vol. 1233 (pp. 54-65). doi:10.1007/ 978-3-030-51999-5_5

Holmberg, L., Davidsson, P., Olsson, C. M., \& Linde, P. (2020). Contextual machine teaching. In IEEE International Conference on Pervasive Computing and Communications Workshops. doi: 10.1109/ PerComWorkshops48775.2020.9156132

Holmberg, L., Generalao, S., \& Hermansson, A. (2021). The Role of Explanations in Human-Machine Learning. In Submitted.

\section{Related publications, not included in the thesis}

Ghajargar, M., Persson, J., Bardzell, J., Holmberg, L., \& Tegen, A. (2020). The UX of Interactive Machine Learning. In Proceedings of the 11th Nordic Conference on Human-Computer Interaction: Shaping Experiences, Shaping Society (pp. 1-3). doi: 10.1145/3419249.3421236

Holmberg, L. (2018). Human-Technology relations in a machine learning based commuter app. In Interactive Adaptive Learning, CEUR Workshop Proceedings, Vol. 2192 (pp. 73-76).

Holmberg, L. (2019). Interactive Machine Learning for Commuters: Achieving Personalised Travel Planners through Machine Teaching. UITP (International Association of Public Transport) Global Public Transport Summit.

Holmberg, L. (2020). Human in Command Machine Learning - Poster version. In AAAI/ACM Conference on AI, Ethics, and Society, Poster Session.

Holmberg, L., Davidsson, P., \& Linde, P. (2020). A Feature Space Focus in Machine Teaching. In IEEE International Conference on Pervasive Computing and Communications Workshops (pp. 1-2). doi: 10.1109/ PerComWorkshops48775.2020.9156175 
Personal contribution For all included papers and workshop publications above, the first author was the main contributor with regard to inception, planning, execution and writing of the research. 


\section{ACKNOWLEDGEMENT}

I would like to thank my supervisors Paul Davidsson, Per Linde and Carl Magnus Olsson. Also, I would like to thank Romina Spalazzese, Jan Persson and Jose Font for their support and guidance during the yearly followup meetings. During the years many persons significantly influenced this work; in no particular order: Helen Hasslöf, Jonas Löwgren, Maria Hellström Reimer, Inger Lindstedt, Clint Heyer, Sara Lebond, Cecilia Hultman, colleagues, students and friends.

I would also like to thank the Knowledge Foundation and Malmö University, in collaboration with several industrial partners, for financing the Internet of Things and People (IOTAP) research profile that, in turn, made this work possible. I am also grateful for the support from K2, Sweden's national centre for research and education on public transport, that in part financed the public transportation design experiment. Finally, I am in debt to K3, School of Arts and Communication Malmö University, for taking care of me over the years. 



\section{CONTENTS}

ABSTRACT. .................... VII

SAMMANFATTNING $\ldots \ldots \ldots \ldots \ldots \ldots \ldots \ldots$ IX

LIST OF PUBLICATIONS. . . . . . . . . . . . XI

ACKNOWLEDGEMENT. .................. XII

I HUMAN IN COMMAND MACHINE LEARNING 1

1 INTRODUCTION $\ldots \ldots \ldots \ldots \ldots \ldots \ldots \ldots \ldots \ldots \ldots$

1.1 Research questions and contributions $\ldots \ldots \ldots \ldots \ldots 6$

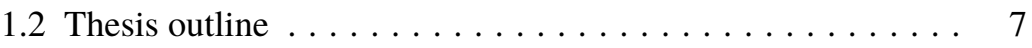

2 DESIGN EXPERIMENTS $\ldots \ldots \ldots \ldots \ldots \ldots \ldots \ldots, 8$

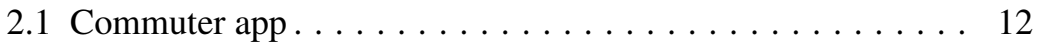

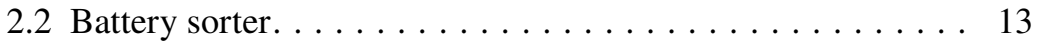

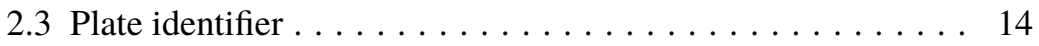

3 BACKGROUND ..................... 15

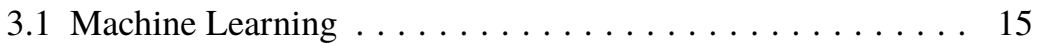

3.2 ML system design process . . . . . . . . . . . . . . 16

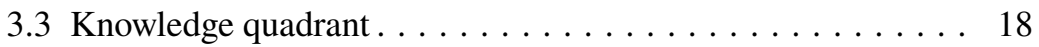

3.4 ML method . . . . . . . . . . . . . . . . . 20

3.5 Explainability, Explicability and Interpretability. . . . . . . 21

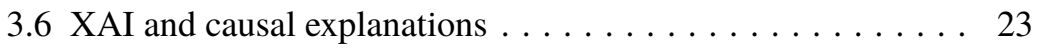

3.7 Mental models . . . . . . . . . . . . . . . . . . . 24

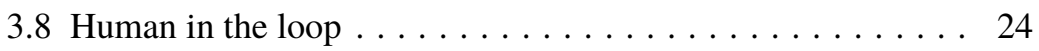

4 METHOdOLOGY ..................... 27

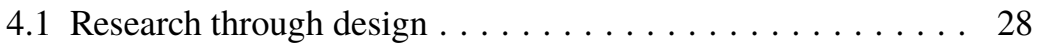

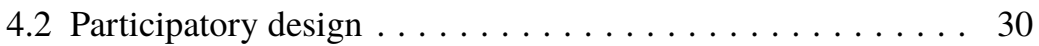

4.3 Design science research methodology . . . . . . . . . . 32

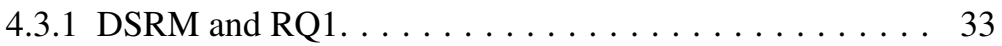

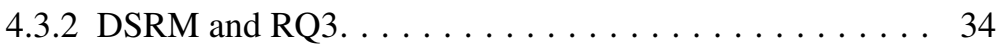

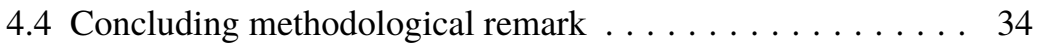

5 CONTRIBUTIONS $\ldots \ldots \ldots \ldots \ldots \ldots \ldots \ldots \ldots \ldots \ldots$

5.1 RQ1: How would a HIC-ML approach alter the ML system

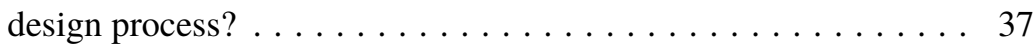

5.1.1 Abstract model for HIC-ML usage . . . . . . . . . . 38 
5.1.2 Design guideline: Feature space . . . . . . . . . . . . . 39

5.1.3 Design guideline: Model space . . . . . . . . . . 41

5.1.4 Design guideline: Training data adjustment . . . . . . . 41

5.1.5 Design guideline: Assessment . . . . . . . . . . . . . 43

5.1 .6 Summary . . . . . . . . . . . . . . . 44

5.2 RQ2: What new kinds of ML systems can be built based on a HIC-ML approach? . . . . . . . . . . . . . . . . . . . 45

5.2.1 Commuter App: Design experiment . . . . . . . . . . 45

5.2.2 Sorting items: Battery: Conceptual . . . . . . . . . 46

5.2.3 Hybrid systems: Plate design experiment. . . . . . . . . 47

5.2 .4 Summary . . . . . . . . . . . . . . . . 47

5.3 RQ3: How can a HIC-ML approach make use of human capabilities combined with ML capabilities to construct causal

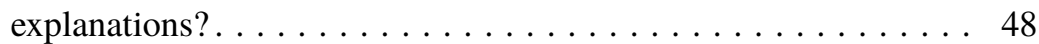

5.3.1 Abstractions and practices. . . . . . . . . . 48

5.3.2 Applied on the commuter design experiment . . . . . . . 49

5.3.3 Applied on the the battery sorter . . . . . . . . 50

5.3.4 Applied on the plate design experiment. . . . . . . 51

5.3 .5 Summary . . . . . . . . . . . . . . . . 52

6 DISCUSSION................... 54

6.1 HIC-ML augmenting human abilities . . . . . . . . . 55

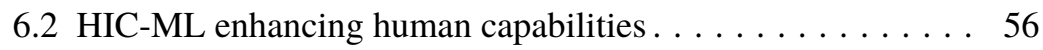

6.3 Mental model and UX. . . . . . . . . . . . . . . . . . 57

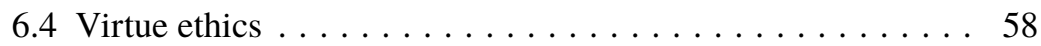

6.5 Limitations of the HIC-ML approach . . . . . . . . . . . 59

6.6 Future work. . . . . . . . . . . . . . . . . . 60

7 CONCLUSIONS. .................. 61

REFERENCES $\ldots \ldots \ldots \ldots \ldots \ldots \ldots \ldots \ldots \ldots \ldots \ldots$

GLOSSARY and ACRONYMS. ............ 69

II PAPERS 77

PAPER 1 - Evaluating Interpretability in Machine Teaching . . 79

Abstract . . . . . . . . . . . . . . . . . . . . . . 79

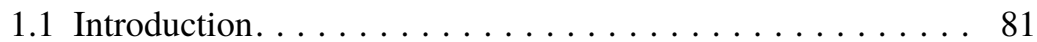

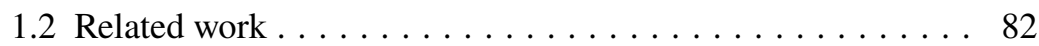

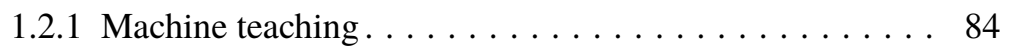

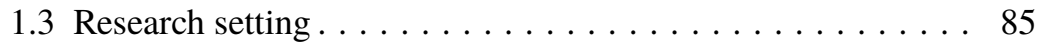




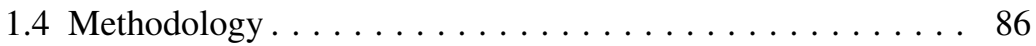

1.5 Result and Analysis $\ldots \ldots \ldots \ldots \ldots \ldots \ldots \ldots$

1.6 Functionally-grounded Evaluation: no humans,

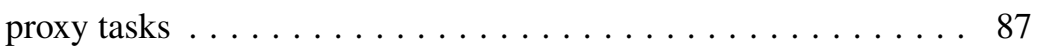

1.7 Human-grounded evaluation: proxy humans, simplified tasks. 88

1.8 Application-grounded evaluation: real humans, real tasks . . 89

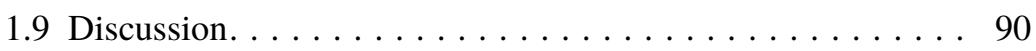

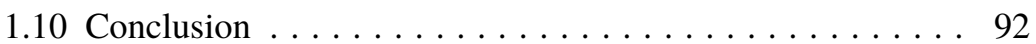

References........................ 93

PAPER 2 - Contextual machine teaching ............ 95

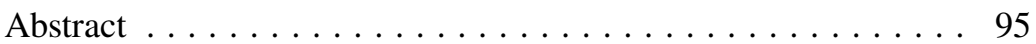

1.1 Introduction. . . . . . . . . . . . . . . . . . . . . . . 97

1.2 Related work . . . . . . . . . . . . . . . . . . . . . 98

1.3 Methodology . . . . . . . . . . . . . . . . . . . . 99

1.3.1 Research setting. . . . . . . . . . . . . . . . . . 99

1.3.2 Recruiting participants for the study . . . . . . . . 102

1.3.3 Participatory generated data. . . . . . . . . . . . . 102

1.4 Analysis and Result . . . . . . . . . . . . . . . . . . . 103

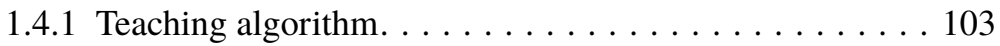

1.4.2 Teaching strategies. . . . . . . . . . . . . . . 104

1.4.3 Prediction in and out of context . . . . . . . . . . 105

1.4.4 Cold start . . . . . . . . . . . . . . . . . 106

1.4.5 Transfer concept to learner . . . . . . . . . . . . . . 107

1.4.6 Teaching Language. . . . . . . . . . . . . . . . . . . 107

1.5 Discussion. . . . . . . . . . . . . . . . . . . . . . . . 108

1.6 Conclusions. . . . . . . . . . . . . . . . . . . . . . . . . . . . . 109

1.7 Acknowledgment . . . . . . . . . . . . . . . . . . 110

References. . . . . . . . . . . . . . . . . . . . . . . . 110

PAPER 3 - The Role of

Explanations in Human-Machine Learning . . . . . . . . . . 113

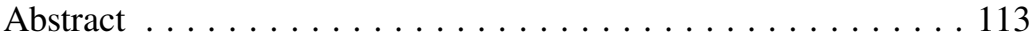

1.1 Introduction. . . . . . . . . . . . . . . . . . . . . . . . . 115

1.2 Related Work. . . . . . . . . . . . . . . . . . . . . . . . 117

1.3 Method . . . . . . . . . . . . . . . . . . . . 120

1.3.1 Research Setting . . . . . . . . . . . . . . 120

1.3.2 Study Design . . . . . . . . . . . . . . . . . . . 123

1.3.3 Data Collection . . . . . . . . . . . . . . . . . . . . 124

1.3.4 Data Analysis . . . . . . . . . . . . . . . . . . . . 124 
1.3.5 Limitations . . . . . . . . . . . . . . . . . . . . 125

1.4 Result and analysis . . . . . . . . . . . . . . . 125

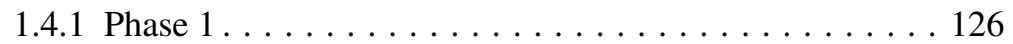

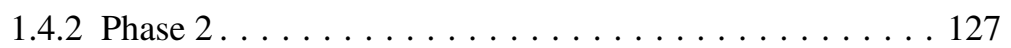

1.4.3 Phase 3: Interview . . . . . . . . . . . . . . . . 127

1.5 Discussion. . . . . . . . . . . . . . . . . 130

1.5.1 Structure of ML Explanations . . . . . . . . . . 130

1.5.2 ML Explanations from a Human Perspective . . . . . . . 131

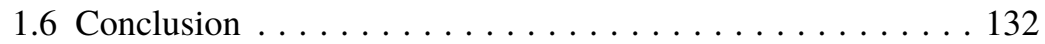

References........................ 133 


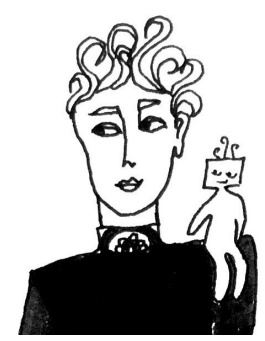

PART I.

\section{HUMAN IN COMMAND MACHINE LEARNING}





\section{INTRODUCTION}

There's a great phrase, written in the '70s: 'The definition of today's AI is a machine that can make a perfect chess move while the room is on fire.' It really speaks to the limitations of AI. In the next wave of AI research, if we want to make more helpful and useful machines, we've got to bring back the contextual understanding.

Fei-Fei Li

At the 2020 edition of one of the most influential Artificial Intelligence (AI) conferences, AAAI ${ }^{1}$, Stuart Russel held a keynote named 'How Not to Destroy the World With AI' ${ }^{2}$. Other prominent and influential speakers were Nobel Memorial Prize in Economic Sciences winner Daniel Kaneman and Turing award winners Yoshua Benigio, Geoffrey Hinton and Yann LeCun, who together held a fireside talk focused on shortcomings of today's $\mathrm{AI}^{3}$. A conference colocated with AAAI-2020 was Artificial Intelligence Ethics and Society (AIES) ${ }^{4}$ that brought up many problematic issues related to AI. For example, how explanations from AI systems can be used to manipulate user trust (Lakkaraju \& Bastani, 2020) or consequences from an idealised approach to algorithmic fairness in a non-ideal world (Fazelpour \& Lipton, 2020). These are some examples of the raising awareness of consequences concerning the usage of AI, other are for example the European Union's proposal for harmonised rules for AI ${ }^{5}$, Stanford University's Human-Centered Artificial Intelligence Institute ${ }^{6}$, Trustworthy Machine Learning Initiative ${ }^{7}$, NYU's AI Now ${ }^{8}$, and conferences like FAccT ${ }^{9}$.

Concerns on potential hazards of uncritical acceptance of the potential

\footnotetext{
${ }^{1}$ https://aaai.org/Conferences/AAAI-20/

${ }^{2}$ https://www.youtube.com/watch?v=QPSgM13hTK8

${ }^{3}$ https://vimeo.com/390814190

${ }^{4}$ https://www.aies-conference.com/2020/

${ }^{5}$ https://digital-strategy.ec.europa.eu/en/library/proposal-regulation-laying-downharmonised-rules-artificial-intelligence-artificial-intelligence

${ }^{6}$ https://hai.stanford.edu/

${ }^{7}$ https://www.trustworthyml.org/

${ }^{8}$ https://ainowinstitute.org/

${ }^{9} \mathrm{https}$ ://facctconference.org/
} 
of AI, without considering the foundational need of addressing also ethical and democratic dimensions of technological development led me to more closely study AI from a contextual user-centred entry point. Using that entry point to study Machine Learning (ML), as design material, exploring its different potential properties and behaviour as humans interact with it, a human in command strategy emerged as a central theme for my research. In this work ML is in focus and is seen as maybe the currently most important sub-field of AI.

Designing for the human in command can be a meaningful starting point for addressing the ethical and democratic concerns mentioned. But it also has potential for creating value in interaction, individualising a use that is often rather general and devoid of context and as such configuring a better and situated fit into people's lives. Having the individual human in command and combining individual knowledge with the potential of ML systems then makes for a user-friendliness in interacting and utilising the, for an ML system, unique teach-learn capabilities. ML systems teachlearn capabilities imply here that a human can transfer knowledge to an ML system and that the ML system can learn and represent that knowledge internally. This approach additionally promotes human learning, meaningmaking and engaged uptake of AI widely also from a mere rational point of view, while at the same time having the ethical and democratic dimension in mind.

Bringing contextual understanding to ML can be approached in several ways, for example, by developing artificial agents that better understand context and humans or, by moving responsibility and control related to the usage of the technology towards human users. By focusing on the latter approach we can increasingly see ML as a design material: a material that needs to be moulded to fit user needs and limitations, a material that makes sense to its users, support society, is user-friendly, create meaning and value and last but not least can be fun to use. This contextual turn in ML is then a logical continuation of the semantic turn (Krippendorf, 2005; Cooper, 1999) and a direct consequence of maturing technology in the ML area. From a design perspective, ML adds not only teach-learn capabilities but also a temporal quality to the more traditional command-action interaction. This temporal quality implies that the ML system can, due to these teach-learn capabilities, over time evolve and change its behaviour. This thesis focus on opening up this design space and put the ML system's teach-learn capabilities into the hands of domain experts and leave it to 
them to subjectively decide in what way this powerful technology can and should create meaning and value.

Vallor (2016), in her book 'Technology and the virtues: A philosophical guide to a future worth wanting', give a philosophical backdrop to the work presented here, in that she proposes that we all need to develop technomoral virtues, virtues that make it possible to navigate the new technological landscape. Examples of traditional virtues, proposed by Vallor, that needs to be fostered and transformed into technomoral virtues are then: honesty, self-control, humility, justice, courage, empathy, care, civility, flexibility, perspective, magnanimity and practical wisdom. Fostering the development of these virtues as technomoral, implies that an individual is in better control of the technology. Virtues, in general, have the strengths of being culturally situated, but still shares similarities between cultures, and are central in the creation and uphold of societies. Vallor names our current state as a state of technosocial opacity, an opacity that implies that we increasingly see the reality through a technological lens that reveals a reality created by algorithms. In this work, the goal is to address parts of this opacity by moving agency in relation to the usage of ML closer to the ones affected by the technology. If we are to develop technomoral virtues related to the use of ML systems, a design approach that transfer agency in the human individual's direction is needed, a shift that positions the user in command of the usage of the ML system.

ML systems trained on huge datasets can surpass human performance within a well-defined domain to, for example, recognise objects in images or playing games. Since these systems are classified as artificial intelligence it is easy to confuse their abilities with humans that have a similar skill level. The central difference is that a human can transfer intellectual skills learned in one domain into other domains (Chollet, 2019). ML systems with similar goals has to rely on humans adding knowledge priors related to the target domain to be able to transfer knowledge from one domain to another. Perhaps more problematic, from a societal standpoint, is that the systems do not, without retraining, adapt to cultural, social and historical shifts and they are incapable of including any non formalised ethical reasoning (HLEG, 2019; Leslie, 2019).

For the discussion that follows, the notions $M L$ expert, domain expert and user are used to denote three central stakeholders in relation to an ML system. An ML expert is a person that can select, build and train an ML model given a problem formulation. A domain expert is a type of user that 
knows, at least partly, the target domain and can, at least partly, evaluate a proposed decision from the ML system. Finally, the user believes that the ML system can add some type of value or meaning. In this work, the output from an ML system is denoted a decision which can take the form of a classification, recommendation, prediction, proposed decision or action. Domain experts and users overlap and are non-static, i.e. a user can become a domain expert and a domain expert can increase their knowledge about the target domain. The ML expert's role is viewed as a craft concerning ML and a person that has a central role in the design team. The notion design team will be used to refer to all competencies needed to design and develop the ML system. Creating tools, terminology, abstractions and guidelines useful for the design team is the focus in the work presented here.

The perspective shift for ML system design envisioned in this work, centres around value- and meaning- creation for human domain experts in command i.e. persons that are responsible for direction, purpose and ethical considerations concerning the usage of an ML system. This type of system is henceforth denoted as Human In Command Machine Learning (HIC-ML) systems. Command is then regarded as a governance principle, a principle that gives the human domain expert authority over the ML system. The HIC-ML approach then implies a human domain expert that governs the system and understands its capabilities as well as its limitations.

In the Human In The Loop (HITL) approach to ML, which is related to HIC-ML, the focus is on the ML model and how human capabilities can be used to optimise the ML model's training process (Settles, 2010; Fails \& Olsen, 2003; Simard et al., 2017). HIC-ML instead takes the form of a partnership between human and technology. This is not new, the perspective that adds to existing work is an increased focus on designing the system so the teach-learn capabilities of ML systems serve the domain expert and position them in command over these capabilities. In some sense, this approach positions a Machine In The Loop (MITL) instead of a human.

\subsection{Research questions and contributions}

This thesis work originated from a curiosity around what happens if the design focus for an ML system is directed towards subjectivity and contextuality. This initial curiosity boiled down to the following research questions: 


\section{RQ1: How would a HIC-ML approach alter the ML system design}

process? This question concerns the craft of constructing HIC-ML systems compared to constructing traditional ML systems. The question is answered by presenting an abstract model of the usage and guidelines that aim to aid design teams in the task of creating HICML systems (See Section 5.1).

RQ2: What new kinds of ML systems can be built based on a HICML approach? This question points towards characteristics for domains where the governance principle command can add qualities for the user(s). Three design experiments show the versatility of the HIC-ML approach and points towards additional areas where the approach can be useful when designing new products and services (See Section 5.2).

RQ3: How can a HIC-ML approach make use of human capabilities combined with ML capabilities to construct causal explanations? Explaining decisions is a central challenge in any complex ML system that should not be trusted blindly. To answer the question the vocabulary, structure and rigidity of scientific explanations are selected and used to form a design path useful to create explanations that bridge the gap between human capabilities and ML capabilities. Additionally, a trust quadrant is presented as a tool useful to understand the the validity of explications produced by the ML system (See Section 5.3).

\subsection{Thesis outline}

This thesis is divided into two parts, the first part present and discuss HIC-ML as a perspective shift for ML system design, the second part present articles that provides a base for the work. Part I is as follows: Section 2 present the design experiments that will be discussed in the later sections. Section 3 situates the presented work in the research field. The methodological approach is then presented (See Section 4). The contribution section follows and is divided into sub-sections in relation to the research questions (See Section 5). The discussion section then situates the contributions in relation to the field (See Section 6). In the conclusion section, central results are summarised and research areas that need further attention are discussed (See Section 7). A list of glossary and abbreviations used follows. 



\section{DESIGN EXPERIMENTS}

In this section, the three design experiments are described. They are presented early since they will be used to exemplify in the sections that follow. They should not be regarded as results, instead, they are used in line with the Research through Design (RtD) research methodology as design experiments central in a knowledge generation process that aims to explore possible futures (See Section 4) (P. Stappers \& Giaccardi, 2017).

Additionally, the design experiments function as boundary objects (Star, 2010) in line with the qualities of being 'temporal, based in action, subject to reflection and local tailoring'. A quality emphasised in this work is the selection of domains for the design experiments that are mundane and can be discussed both from an ML expert's and domain expert's perspective and ideally be understood by persons that are not domain experts. If these discussions have concrete outcomes it will be possible to express them so they can be understood and discussed by an interdisciplinary design team. Central in the RtD approach used is a curiosity around consequences that follows when the teach-learn capability of ML is treated as a design material that can be subjectively shaped by a domain expert. This research framing has been generative in the sense that the selection of design experiments for this work is a result of upcoming questions during an incremental knowledge production process.

In line with the RtD approach, the three design experiments helped sharpen and develop the research questions. RQ1: 'How would a HIC-ML approach alter the ML system design process?' and the commuting app evolved together based on practical experiences and comparison between a traditional ML design process and a design process as a consequence of a HIC-ML approach. RQ2: 'What new kinds of ML systems can be built based on a HIC-ML approach?' is connected to the battery sorter that explores how teach-learn capabilities in the hands of domain experts can augment human abilities in a context with constant data drift (new brands and markings of batteries). The intriguing black-boxing of reasons for a decision in complex ML systems then evolves into the plate design experiment that focuses on decisions in a system that enhances human cognitive abilities and RQ3: 'How can a HIC-ML approach make use 
of human capabilities combined with ML capabilities to construct causal explanations?'. Here an ethical demanding setting was avoided to make the study feasible. As a consequence trust and trustworthiness is not discussed to any large extent since these concepts are strongly related to the severity of consequences of erroneous decisions (HLEG, 2019).

To get started on the research endeavour teach-learn ML capabilities was added to an existing commuting app. This design experiment was selected in line with the RtD methodology (Herriott, 2019) as a designerly way of finding out and explore HIC-ML. The initial research question used to explore the area was: 'Which consequences emerge, technically and from a usage perspective, if commuters teach their commute patterns to an ML model so the app can present their upcoming journey when the app is brought into focus?'. My curiosity was directed towards the subjective experience of being in control of the teach-learn process. One characteristic of the commuting app is that the knowledge domain is personal, implying that the commute patters taught to the ML model only matches the individual commuter's commute patterns. There is therefore not any motivation to share trained ML models between commuters or downloading some general pretrained model, since there will be limited overlap in commutes with other commuters. A natural expansion of the research was then to search for a design experiment in a shared knowledge domain as a complement to the personal knowledge domain the commuting app represent.

Inspired by the work of Lindvall et al. (2018) in radiology I tried to find a mundane and accessible domain that shares challenges with radiology, but devoid of sensitive data and dependence on highly specialised knowledge. The choice fell on a battery sorter (See Section 2.2) since it represents a shared knowledge domain and answers to similar demands as radiology: it is dependent on a physical device and uses details and subtle information in images to promote decisions. Additionally, there are experts in the recycling area as well as users which consequently calls for a governance system that includes privilege rights with different access levels. Building a fully functional design experiment was not within the scope of the research, the design experiment is instead conceptual and in addition used to make feasibility tests related to the selected machine learning method. A simple neural network that reasonably well classifies batteries based on 2-3 images of each battery was used for this design experiment. The blackbox quality of a neural network is intriguing and there is no simple way to know which part of the image the network base a proposed decision on. 
Insights like these lead to questions around explainable black box systems and hybrid human-machine system that can enhance human capabilities if they can be trusted.

The plate design experiment answers to these challenges since the ML system can, from a cognitive perspective, possess abilities unreachable for humans. As a consequence, explanations are needed to understand why a decision is made and eventually, what it takes to trust an ML system with these abilities not reachable for humans. The problem domain targeted by the plate design experiment is open-ended and there are many interesting questions and future research paths to explore. 


\subsection{Commuter app}

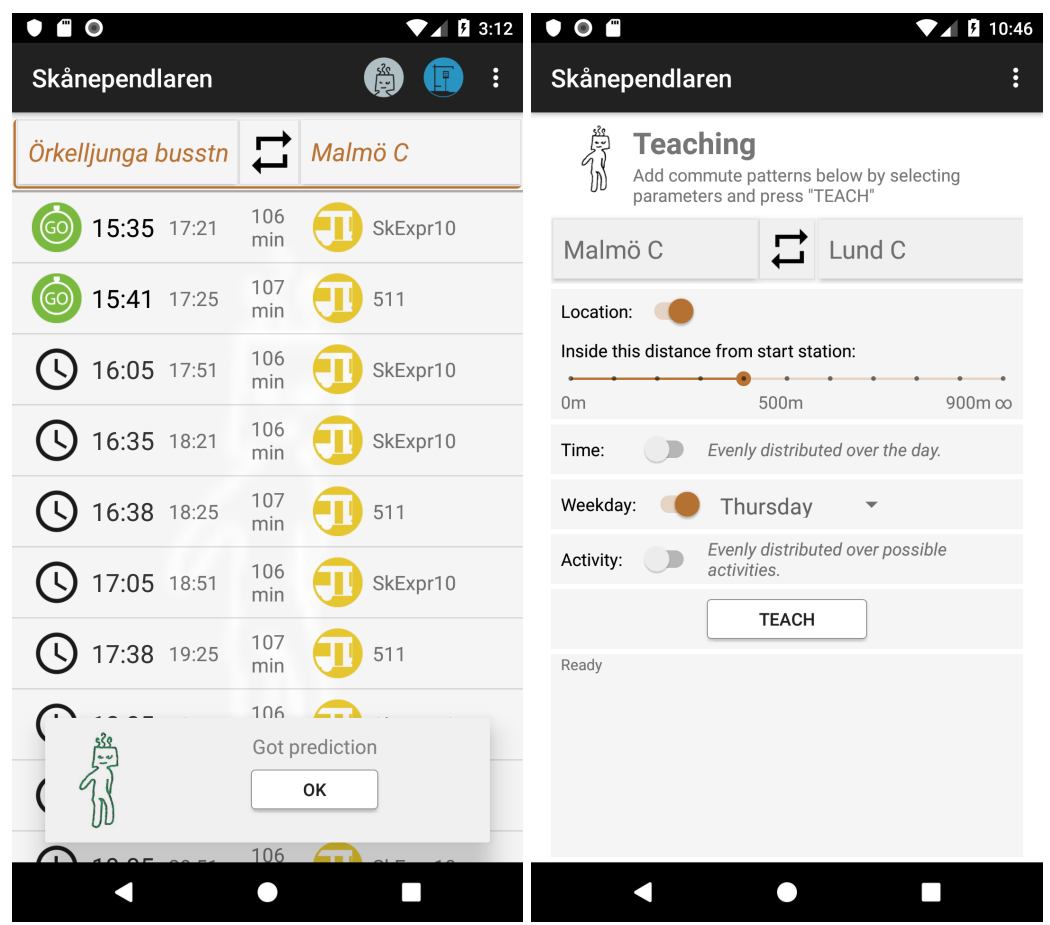

Figure 1: Commuter design experiment. To the left, a prediction arrived and the app collected relevant journey information from the service provider. To the right, the teaching interface is shown with available features.

This app allows a commuter to teach an individual ML model so it predicts upcoming journeys. The design experiment is discussed in Paper 1 and 2. The user interface for predictions can be seen to the left in Figure 1 and the teaching interface to the right in Figure 1.

A journey is proposed when the app is brought into focus on the mobile phone, the proposed journey depends on the features: time, weekday, location and activity (still, walking, running, in vehicle). The app only and always promotes the journey predicted with the highest class probability. Details outlining the design from a more technical perspective can be found on the project's website ${ }^{10}$.

The target domain for the design experiment is commuting treated as a personal knowledge domain e.g. the commuter is seen as the only expert regarding their commute patterns.

${ }^{10}$ https://github.com/k3larra/commuter 


\subsection{Battery sorter}

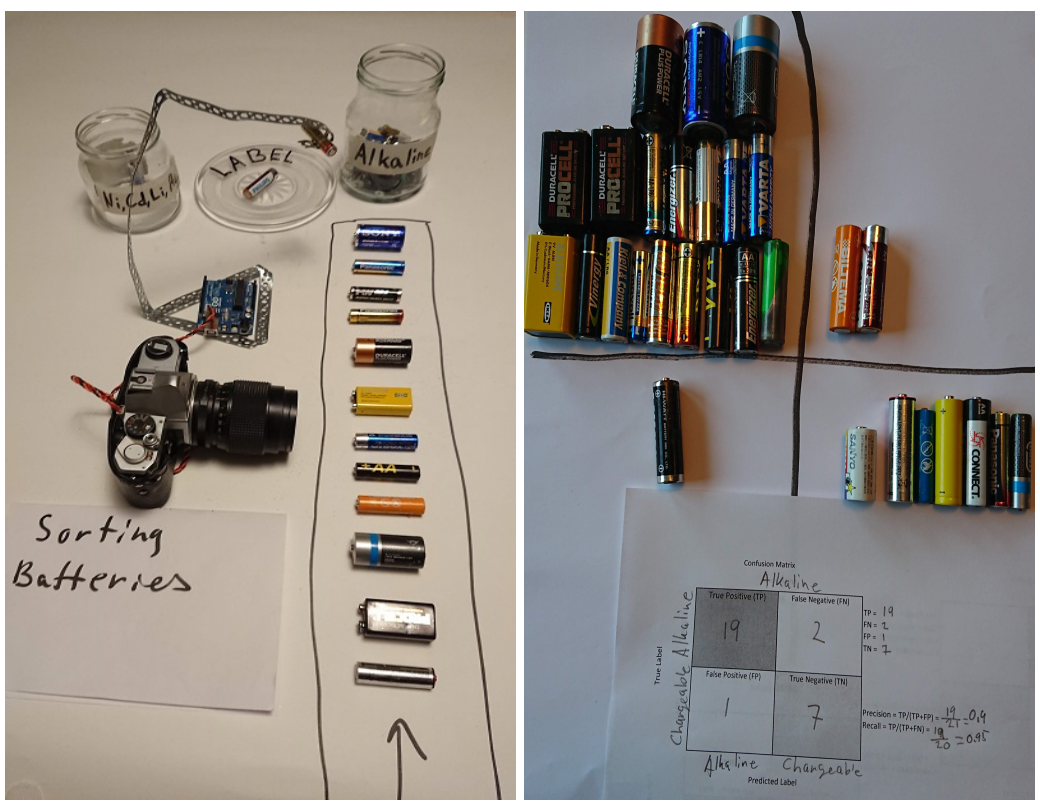

Figure 2: To the left, the conceptual battery sorter. To the right, actual battery sorting result assessed using a physical confusion matrix.

This design experiment is the least developed of the three and exists only on a conceptual level, as seen in Figure 2. The design experiment makes it likely that by using transfer learning (ResNet34) and retraining the network using a few high-resolution images of each battery, it is possible to sort batteries in two categories with reasonable accuracy.

In the conceptual design experiment, to the left in Figure 2, the batteries are transported on an imagined conveyor-belt in front of a camera that sends imagined images to a conceptual computer that classifies the images and uses the conceptual robotic arm to sort the battery as Alkaline or $\mathrm{NiCd}$. In the centre, batteries are placed for labelling based on low class-probability.

Assessment of the sorting outcome was visualised using a physical confusion matrix, as seen in the right image in Figure 2. Details outlining the design experiment can be found on the project's webpage ${ }^{11}$.

The target domain for the design experiment is battery sorting, the domain is treated as a shared knowledge domain e.g. contextual domain knowledge is shared between users and recycling specialists.

${ }^{11}$ https://github.com/k3larra/interactiveML/blob/master/batteries_AL.ipynb 


\subsection{Plate identifier}

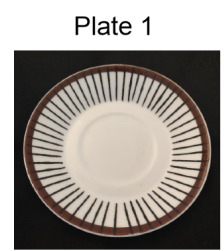

Plate 2

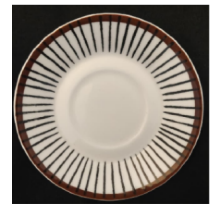

Plate 3

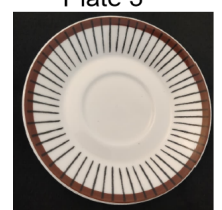

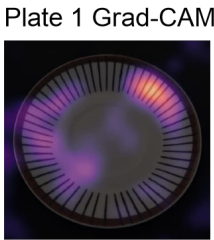

Plate 2 Grad-CAM

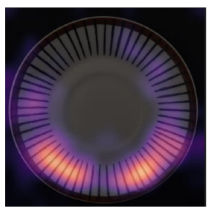

Plate 3 Grad-CAM

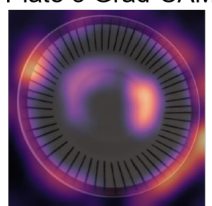

Plate 4

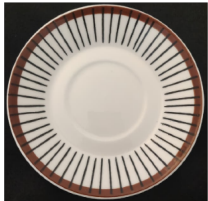

Plate 5

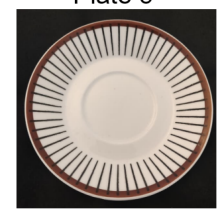

Plate 6

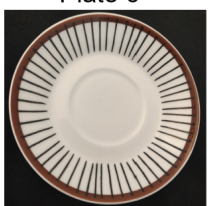

Plate 4 Grad-CAM

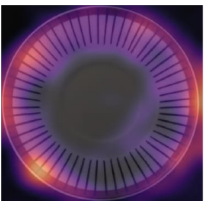

Plate 5 Grad-CAM

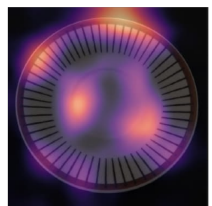

Plate 6 Grad-CAM

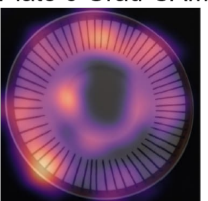

Figure 3: Original images to the left and generated Grad-CAM images to the right.

The porcelain plates shown in Figure 3 are the reference objects in this design experiment. They are six hand-painted plates from the Spisa Ribb series by the Swedish porcelain factory Gustavsberg. Spisa Ribb features bone china with a transparent glaze, a brown edge and black vertical lines. The six plates used in this study were produced before 1974 when the plates were still painted by hand. From 2003 a new series is produced, called Ribb, where the decor is printed and not hand-painted.

Around 40 relatively high-resolution images per plate were used to train a model based on a pretrained ResNet50 model. In Figure 3 visual explications are shown in relation to the plates. These explications lift out salient factors that contrast a specific plate from the other plates. Additional information for reproducibility, code and images can be found in Paper 3 and on the project's website ${ }^{12}$.

The target domain for this design experiment is not predefined to the same extent as for the other experiments. A domain expert is anyone interested in comparing and distinguishing similar objects that can be represented in images. The target domain can be shared between a group with similar interest or be personal.

${ }^{12} \mathrm{https} / /$ github.com/k3larra/spisaribb 


\section{BACKGROUND}

The two first sections that follows contains an overview of the part of the AI landscape this work focuses on. In the following section, the knowledge quadrant is introduced as an abstraction focusing on the relation between the domain expert's knowledge and the ML model's knowledge. Implications of unintelligible ML models are then discussed as an introduction to the section that discusses explicability and scientific explanations. On this follows sections that lift out research related to explainable AI (XAI), mental models and HITL. HIC-ML are in these sections situated in relation to the research areas discussed.

\subsection{Machine Learning}

In this section, the view on and the terminology that will be used throughout this thesis concerning ML will be presented. The acronym AI is used sparingly since intelligence as a concept is not well defined and many aspects of AI research aims to replace or reproduce human intelligence, which is not the goal in this work. Instead, ML is in this work viewed as a sub-field of AI and the aim for this work is to explore ML as a design material that can augment and/or enhance human capabilities, similar to our use of hammers, pens, binoculars, microscopes and watches. The notion $M L$ model denotes a computational resource that can be trained to represent knowledge. The notion $M L$ system includes the ML model plus the computational resources needed to handle input data and present promoted decisions.

To train an ML model you need labelled training data. Labels are the correct answers or ground truth; for the battery sorter, one image of a battery is denoted an example of the input data that in turn is part of the training data that consists of many examples/images. The label for the image is then either Alkaline or $N i C d$. The image is seen as raw input data even if it is restricted by resolution and colour depth. Raw input data then refers to, for example: text, images and sound. They are raw in the sense that individual letters, pixels or frequencies carry little information on there own instead meaning is created in relation to adjacent information. In the commuter app, input data consist of five engineered features: latitude, 
longitude, time, day and the more experimental and outlier feature, the commuters activity (walking, still, running, in vehicle). The features are denoted engineered if they are selected by a system designer to represent an aspect of reality, often independent of the other features, as the features selected for the commuter. Input data will be named, either as, in the commuter design experiment, examples composed of features or as images if that is the format. A labelled example used for training in the commuting design experiment then consists of the contextual features and a specific journey as a label. For images, the notion of features becomes in-explicit and, as a simplification, a human and a neural network uses the same raw data (the whole image) to create internal representations useful to classify objects in the images. Once the ML model is trained it can be used to promote decisions. If the model is exposed to an unlabelled example it will find the label or a numeric output that matches the example best. This is denoted that the ML model generalises in relation to a specific example, that ability, combined with training the model, is the most distinctive property an ML model has. The output or promoted decision can then be continuous numbers or a classification, as in the design experiments in this work.

\subsection{ML system design process}

This work focuses on ML model training as an iterative process where a domain expert is responsible and in command of direction, purpose and ethical considerations concerning the usage of the ML system. None of these three considerations is in this work explored to their full extent and especially the ethical considerations are only seen as a consequence of the HIC-ML approach. RQ1 is formulated as 'How would a HIC-ML approach alter the ML system design process?' and aims to differentiate the process of designing a HIC-ML system compared to a typical ML design process.

As a typical process, the nine-stage machine learning workflow identified by Amershi et al. (2019) is used. The stages are presented as a linear process to deploy a product, the workflow is outlined in Figure 4. In reality, it is not a strictly linear process and, for example, iterations between model training and feature engineering are very common. The stages model evaluation and model monitoring loop back to any of the previous stages if requirements are not met and if the trained model not meets expectations after deployment. Dataset shift (Moreno-Torres et al., 2012), i.e. coping with changes in the deployment context not anticipated in the requirements 
can be highlighted as a central reason for looping back from the model monitoring stage.

The HIC-ML approach does not solve any challenges embedded in the stages, instead, it adds a layer in which design decisions has to be taken by the design team on which parts and to what extent domain experts can benefit from being in control over a specific stage. This transfer of gravity then surfaces challenges that need to be addressed and presented to a domain expert in an understandable fashion. For example, selecting and labelling training data calls both for effective interfaces that can handle a large amount of data, for example, images (Amershi et al., 2011), and methods for addressing and evaluating cold-start issues related to the need for enough training data so the selected ML method can learn (Banovic \& Krumm, 2017; Konyushkova et al., 2017).

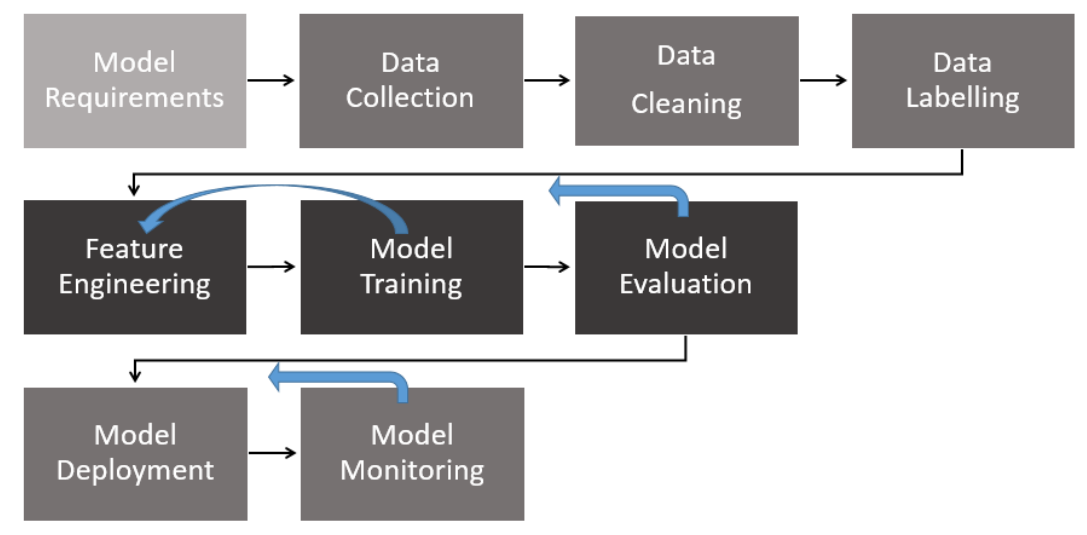

Figure 4: ML workflow identified at Microsoft by Amershi et al. (2019). Feedback loops exists between many consecutive steps and it is especially common that model training loops back to feature engineering, as indicated by the arrow. Model evaluation and model monitoring are somewhat special in that they feed back to any previous stage.

HIC-ML adds an additional design layer to this model, and opens up for a new kind of ML system where the main goal is expressed in terms of value and meaning creation for a domain expert. The main difference being that the design team transfers teach-learn capabilities of the ML system, to some extent, into the hands of domain experts. This then imply a designerly thinking related to temporality, that the system's knowledge representation will change over time, resulting in that it behaves differently over time. The behaviour is governed by the the domain expert who, by teaching the system, control what it learns. 


\subsection{Knowledge quadrant}

A knowledge quadrant (See Figure 5) (Munro, 2021; Chollet, 2019) is in this work used to discuss how the ML system's knowledge relates to the domain expert's knowledge. The labelled examples used as training data are denoted Known Knowns and the unlabelled examples that the ML system promotes labels (decisions) for are denoted Known Unknowns. Known Knowns and Known Unknowns represent the target domain defined and delimited by a problem formulation. The examples then consists of data (for example an image) that represents some aspect of the entity (for example a battery) the ML system promote decisions for.

\section{Knowledge Quadrant}

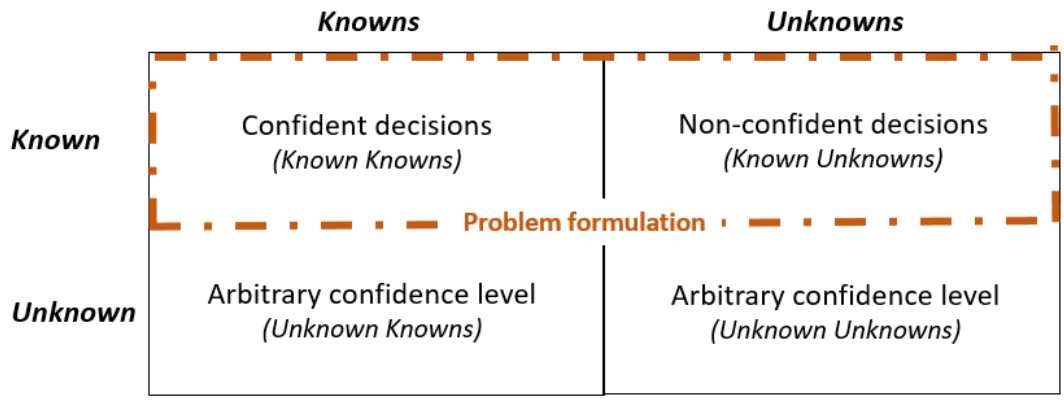

Figure 5: Knowledge quadrant. The quadrant displays the target domain for the HIC-ML system. The top row delimits the part of the domain the ML model is trained to promote decisions for. The bottom left rectangle contains domain knowledge known to the domain expert but unknown to the ML model. The bottom right rectangle contains domain knowledge unknown both to the ML model and the domain expert.

Using the battery sorter as an illustration: battery entities that arrive to be sorted, of a brand the ML model has been trained to classify, belong to the Known Unknowns category and the sorter, if properly trained, should be able to label them correctly. The battery sorter uses images as data to represent the individual entities/batteries. A worn, but known, battery with damaged decoration will be classified with lower confidence than the same type of battery in mint condition. Unknown Knowns are then part of the target domain and known to the domain expert, but for one or another reason the domain expert has decided that these entities are outside the problem formulation and consequently the ML model is not trained promote decisions for them. Unknown Unknowns are entities part of the target domain but unknown to the domain expert and the ML model. 
Again using the battery sorter and delimiting the target domain to batteries: Unknown Knowns are batteries of a brand the ML model not has been trained to classify. Unknown Unknowns are, for example, NiCd batteries that for some reason has the same external design as a Alkaline battery. The confidence level for batteries outside the problem formulation (Unknown Knowns and Unknown Unknowns) will depend on how similar they are to the batteries the model is trained to classify (Known Knowns). This imply that confidence level cannot be the only measurement used to separate batteries not part of the problem formulation from those that can be correctly classified.

Chollet (2019) relates to the top row in the knowledge quadrant as the type of AI systems that currently dominate the AI area, these systems aim for local generalisation and robustness within a domain. Given a problem formulation machine learning experts can optimise accuracy for the Known Unknowns examples so the model generalises well for examples included in the problem formulation. Munro (2021) uses the knowledge quadrant slightly different than Chollet (2019) and relate the quadrant to Human-inthe-Loop (HITL) ML and as a tool that can be used, by an ML expert, to inquire into data that belongs to each rectangle.

Generally, in traditional ML, the focus is on the top row and sometimes the category Unknown Unknowns attracts attention to find outliers in relation to the problem definition (Attenberg et al., 2015; Lakkaraju et al., 2017). The work presented in this thesis is closer in line with Munro (2021), but a human in command is envisioned, a human that is responsible for the problem formulation and understands the domain but has, contrary to Munro (2021)'s ML expert, no expert knowledge in ML. The domain expert can in HIC-ML, if the ML model has the capabilities, adjust the problem definition, for the ML model, by interchanging labelled data between the categories Unknown Knowns and Known Knowns and retrain the model. The work presented in Paper 3 focuses on the gap between the domain expert's knowledge and the ML model's knowledge i.e. the domain expert's understanding concerning the difference between the top row (problem definition) and Unknown Knowns in the knowledge quadrant. For example, in a classification setting, the design team can design a system that supports scientific insights related to the domain (Roscher et al., 2020). This since the domain expert knows the difference between examples that belongs to the domain (top row) and examples outside of the domain (Unknown Knowns) they can, as a consequence, evaluate decisions 
differently. For examples within the problem formulation they can learn more about variations of entities that belong to the same class and for entities outside of the problem formulation they can evaluate the entity's similarity with classes within the domain.

\subsection{ML method}

In this work, raw input data in the form of images are used as training data for the battery sorter and the plate design experiment and, for a domain expert, understandable engineered features are used for the commuter (time, location, day, activity). The domain expert and the trained ML model then relates to the same input data and an explanation of a promoted decision can be produced based on the image data or the features. If a neural network is used, as in the design experiments, it is hard to conceptualise how the knowledge is represented internally in the ML model. The knowledge is black-boxed to human understanding and there does not exist an intelligible relation between the input features and the promoted decision.

In line with Lipton (2016) this work adheres to the view that it primarily the complexity of the problem that results in model black-boxing and not so much the choice of algorithm/method. As discussed in more detail in the next section, explanations in an ML context (Gilpin et al., 2019; Tjoa \& Guan, 2019; Lakkaraju et al., 2016; Camburu, 2020) does not hold as explanations in a human context, for more complex systems. In an ML context, the focus often is ML model centred and aims to connect input data with a promoted decision instead of, as in this work, focus on human understanding. In ML research the notion of explanations often implies, from a social science perspective, causal attributions. These causal attributions then explain the relation between input and output and leave to a human to interpret and reformulate them in relation to the real world (Miller, 2019; Hilton, 1990). This is discussed in more detail in Paper 3.

During training, neural networks create internal layered representations of the knowledge in an inductive fashion (Lecun et al., 2015). There exists a variety of methods that aims to summarise the reasons for a decision, both model-agnostic and model-specific (Belle \& Papantonis, 2020). These methods produce what in this work is defined as causal attributions i.e. they ascribe a phenomenon to its origin by connecting input data with a decision. As discussed in more detail in Section 3.5 these causal attributions are static and do not account for or adapt to the human 
explainee's cognitive capabilities or domain knowledge. Related to the knowledge quadrant described in Section 3.3 causal attributions ascribes phenomena to their origin inside the problem formulation.

For all design experiments used in this work, a neural network is selected as the ML method, since it guarantees that the internal knowledge representation in the ML model will be black-boxed. This decision directs the research towards complex systems and the goal of moving agency in relation to these systems in the domain expert's direction. For less complex problems transparent models can be used, these type of problems are not addressed in this work even if a HIC-ML approach can be used also in these cases.

\subsection{Explainability, Explicability and Interpretability}

A taxonomy and guidelines for Trustworthy AI are defined by the European Commissions' Independent High-Level Expert Group on Artificial Intelligence (HLEG). There are other guidelines both from standard organisations and companies (Jobin et al., 2019) but the EU one is chosen here since it is independent work and culturally relevant. In the HLEG (2019) document, a Trustworthy AI system has three necessary components that should work in harmony: lawful, ethical and robust. AI systems then have to comply with the law and follow ethical principles to be robust, both from a technical perspective as well as a societal perspective. In that work, the term explicability is used and seen as crucial to build and maintaining trust in an AI system. Demands on explicability are then dependent on the context and severity of consequences.

In this work, explicability is defined as capable of being explained by a domain expert. From the definition made here, an explicable decision can be understood using the domain's ontology and hence by a domain expert (This is further discussed in Paper 2). This, in some sense, situates explicable ML systems between interpretable ML systems, that can be explained by humans with sufficient but unspecified knowledge, and explainable ML systems, that does not put demands on the human that are expected to understand the decision. In this work, the focus is then on an ML system that creates explications and brings causal attributions forward to a domain expert as insights and evidence for a decision.

In Paper 3 theories around scientific explanations in human-only contexts are applied to the plate design experiment to lay bare what type of knowledge we can expect from the explications created by an expli- 
cable ML system. Theories around the structure of scientific explanations (Overton, 2012) is there combined with types and forms of explanations (Miller, 2019; Newcomb \& Heider, 1958) into a coherent unit which makes it possible to discuss causal explanations in a generative fashion. In line with Hilton (1990) explanations are seen as a negotiation process between an ML-based explicator and a human explainee. Connecting scientific explanations and human explanations are done since they both aim to answer a why question. This delimits the type of explanations we can expect to produce to those that build on causality and it implies expectations that the human explainee in some sense understand and accept the scientific format and vocabulary connected to the explanation.

To Hilton (1990) a causal explanation in human only contexts are given: implying that someone explains something to someone. Causal explanations then take the form of a conversation often initiated by the explainee asking a why question. Causal attributions produced by the ML system are instead static and aims to ascribe phenomena to their origin. In this work, the focus is on causal explanations and a type of conversation, or dialog sequence, in which a domain expert actively can select causal attributions to understand a promoted decision. Causal attribution is then the selected format used in this work in order to make the ML system explicable in line with the definition of explicability made above.

Overton's model (See Figure 6) covers the structure of scientific explanations and uses vocabulary connected to scientific explanations (Woodward, 2019) to bring rigour to the discussion. This opens up for a discussion around what we can expect from machine explications (Se Paper 3). According to Overton (2012) scientific explanations are built using the following categories:

- Theories: sets of principles that form building blocks for models.

- Models: an abstraction of a theory that represents the relationships between kinds.

- Kinds: an abstract, universal class that supports counterfactual reasoning.

- Entities: an instantiation of a kind.

- Data: statements about activities (e.g. measurements, observations).

Central in Overton's work is to define a core relationship between two categories that the scientific explanation targets. For the battery sorter, 
the core relationship is between two kinds alkaline and $\mathrm{NiCd}$ and data represented by images of batteries. In the plate design experiment, the core relationship is between entities, represented by the individual plates and data in the form of images. Contrastive reasoning answers the question of why A instead of B and implies, in the plate experiment, comparing two entities: for example, for the plate experiment, lifting out several contrastive attributions. Contrastive attributions are then attributes that are not unique but comparably different: for example, again, for the plate experiment, lines that are narrower, closer, longer, etc.

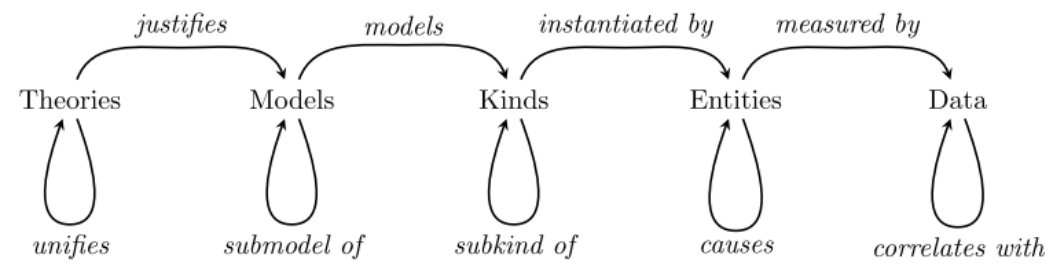

Figure 6: The five categories and four primary relations that builds scientific explanations (Image used by permission from author Overton (2012)).

\subsection{XAl and causal explanations}

The established acronym XAI (eXplainable Artificial Intelligence) is used as an umbrella term for a field that aims to make AI systems understandable for humans. In the area of XAI causal attributions and causal explanations are often confused (Miller, 2019). When models are relatively small, the features understandable and the algorithm transparent the system can be explicable by design (Lipton, 2016). In other cases, explications can be constructed using methods that lift out salient factors. These methods can have a local or global scope and often aims to create local surrogate models to explain individual predictions (Gilpin et al., 2019; Lipton, 2016; Lakkaraju et al., 2019; Doshi-Velez \& Kim, 2017; Lundberg \& Lee, 2017; Ribeiro et al., 2016; Gunning, 2017; Ribera \& Lapedriza, 2019; Kulesza et al., 2015). Global scope refers to methods that approximate the behaviour of the model in the complete feature space and local refers to approximations of decision behaviour in the vicinity of a proposed decision. Linear models that depend on a small subset of features or decision trees with few branches and levels can then, for example, be used to create surrogate models since these models can be intelligible in their entirety.

Interpretable decisions, explications and explanations can be presented 
for a user in a variety of formats, for example, as text, visualisations or as examples and counterexamples (Lipton, 2016). Selecting a suitable format is a design question that depends on, amongst other more technical factors, target domain and context.

Several recent surveys give an indication of the research activity in the area (Biran \& Cotton, 2017; Mueller et al., 2019; Adadi \& Berrada, 2018; Tjoa \& Guan, 2019; Guidotti et al., 2018; Carvalho et al., 2019; Gilpin et al., 2019; Barredo Arrieta et al., 2020) as well as approaches to define taxonomies for the rapidly developing area (Barredo Arrieta et al., 2020; Abdul et al., 2018) of XAI.

\subsection{Mental models}

The concept of mental models will be used to relate to the model a domain expert creates internally in their head during usage, a model that encompasses the HIC-ML system's capabilities. Norman (1983) describes this conceptualisation as 'In interacting with the environment, with others, and with the artifacts of technology, people form internal, mental models of themselves and of the things with which they are interacting. These models provide predictive and explanatory power for understanding the interaction'. The mental models then need to be functional for the domain expert's purpose but not technically accurate, they can be incomplete and the domain expert will modify the mental model over its lifetime. My work argues that the use of mental models in HIC-ML is of importance since the system has a unique usage and training history that is not easily captured and documented. As discussed above a global and complete understanding of the knowledge encompassed by the ML model will often not be possible.

\subsection{Human in the loop}

The role and the degree of end-user involvement in ML training are especially discussed when manual labelling is expensive, the deployment context is non-static or to address different kinds of data drift. Involving users can also mitigate cold start problems or be used to train an ML model towards some goal that is not easily quantified (Fails \& Olsen, 2003; Settles, 2010; Amershi et al., 2014; Simard et al., 2017; Rahwan et al., 2019; Zhu, 2015; Zhu et al., 2018).

In Active Learning (AL) the ML system takes initiative and asks questions to the user if some predefined learning goal is not met. This can, for 
example, be low class probability for a prediction. In many cases, there is a budget concerning, for example, annotation cost that has to be balanced with respect to the learning goal. The annotator is here typically seen as an oracle that is always correct and available (Fails \& Olsen, 2003; Settles, 2010). From the HIC-ML perspective AL can be useful in intensive labelling sessions in order to handle, for example, cold-start. The session will then be initiated and ended by the domain expert. Since HIC-ML builds on the domain experts knowledge and interest the time and effort a domain expert is willing to put in (labelling budget) is decided by the domain expert.

In Interactive Machine Learning (IML) both users of the system and the system can take initiative and signal that the system does not work as expected (Amershi et al., 2014; Boukhelifa et al., 2018; Kulesza et al., 2015). Here UX design becomes central since the human counterparts need insights related to the training process to intervene (Dudley \& Kristensson, 2018). For an HIC-ML approach this approach is interesting since the system can draw attention to labelling errors based on low accuracy for a promoted decision or that a domain expert, for example, can add more training data to balance classes.

In Machine Teaching (MT) there are two strands, one that primarily sees the human teaching a machine as central (Simard et al., 2017; Meek et al., 2016; Ramos et al., 2020) and one that envisions that the roles can shift and machines that teach humans is a possible setting (Zhu, 2015). In Interactive Machine Teaching (IMT) (Ramos et al., 2020) the focus is on systems that can augment human capabilities and therefore on how to support knowledge transfer from a human domain expert to a model. These different approaches to HITL, especially the MT perspective, are discussed in more detail in Paper 1 and Paper 2. A HIC-ML approach has similarities with an MT approach, the difference being that the governance principle imply that HIC-ML is not confined to any specific ML model training approach, instead the focus is on usability of the HIC-ML system. This widened focus treats ML as a design material that can be designed to presents itself to the domain expert as an artefact that over time can be taught and that can learn.

A related area is robot learning (Chernova \& Thomaz, 2014; Cakmak et al., 2010; Ravichandar et al., 2020) that shares challenges with this work since it in many cases build on HITL approaches. The techniques used can often conceptually, except for Learning from Demonstration (LfD), 
be reduced to AL, IML and MT. Many areas in robot learning parallel and extend work in HITL and points towards interesting, and in this work unexplored, future research paths for HIC-ML.

The HIC-ML approach differs in the sense that that the focus is, not on the ML model or the technology, instead value and meaning creation for the human domain expert is in focus. Prediction accuracy and generalisation are then still important base requirements but not the only focus for the domain expert since the domain expert envisions a learning goal for the HIC-ML system that is subjectively defined. This implies that the usefulness of both traditional learning strategies, like supervised and unsupervised learning, and training approaches involving humans, like AL, IML and MT, depend on the domain expert's learning goal, knowledge and available resources. This perspective change places the machine, instead of the human, in the loop and implies that selecting appropriate training approaches, as Al, IML and MT, becomes a part of HIC-ML design and thus a responsibility of the design team.

In one setting a pretrained model can perhaps directly fit the needs whilst in other settings, extensive training involving many persons with different skills can be needed. Using the battery sorter as an example; an initial MT session could be followed by an AL setting where low accuracy initiates a call for manual labelling or inspection. For the commuter experiment, an initial MT session could be followed by IML so features connected to low accuracy predictions can be presented by the system and, for example, timespans can be adjusted to match changes in commute patterns. An initiative from the commuter can then be deleting and adding commutes. For the plate prototype, that enhance human cognitive capabilities, explications come into focus. The app can then be used to identify plates but also, for example, to compare plates in different collections to find similarities. Here an MT approach can be selected for the teach-learn phase whilst usefulness depends on the quality of explications and how they are presented. HIC$\mathrm{ML}$ is then a design layer above training approaches, like AL, IML and MT, in that the design team decides, depending on the domain, when and to what extent an ML model training approach is available and selectable for a domain expert. 


\section{METHODOLOGY}

Curiosity around the impact of subjectivity and contextuality in machine learning development is a key perspective in this work. This leads to questions around design challenges that emerge when a domain expert is in command of training and usage of the ML system. For this type of exploratory endeavour the methodology Research through Design (RtD) (P. Stappers \& Giaccardi, 2017) can fit well, especially if the work centres around design experiments. In RtD the goal is not primarily to produce or improve a product, instead, the goal is to, during the process, produce generative theories and explore possible futures (Gaver, 2012). As a starting point a decision was made to redesign an existing commuter app, so it opened up for commuters to subjectively teach a neural network commute patters. The rigidity and the mundane qualities of the commuting problem forced me to explore ML as a design material. During this explorative process the research questions gradually took form. This relationship between the iterative formation of research questions and experiments is one a characteristic property of the RtD processes.

Predicting journeys for a commuter can be solved using more traditional methods, for example, a solution based on algorithmic ML models such as decision trees or codified logic that infers the journeys from the context. From a utility perspective these approaches are probably better than utilising the teach-learn capabilities of a neural network. For this design experiment the knowledge object selected is not utility, instead, it centres on design challenges related to a situation when a human is in command of the teach-learn capabilities of an ML system. Design challenges exposed relates then both to how HIC-ML approach alter the design process (RQ1) as well as meaning-making related to promoted decisions (RQ3).

The prototype worked reasonably well as a commuting app but had also the qualities of a boundary object (Star, 2010) since it targets a well-known domain that can be understood and discussed by users with different perspectives and background. To widen understanding of use perspectives concerning different commute patterns a Participatory Design (PD) (Robertson et al., 2012) study was conducted. The goal was to reframe the research questions and better understand the HIC-ML approach, 
especially from a temporal usage perspective.

To answer to demands on rigidity the epistemic stance gradually shifted towards post-positivistic knowledge production and formalising the results as an abstract model and guidelines. Design Science Research Methodology (DSRM) (Peffers et al., 2007) was selected as it aims to balance positivistic and interpretivistic epistemic stances. DSRM then in addition to quantitative research opens up for qualitative research and support outcomes to problems not hitherto addressed.

Related to methodology, some notes are made here related to the word Design since it is used somewhat differently in PD and RtD compared to how it is used in Design Science (DS) and DSRM. Design, as used in DSRM, is rooted in engineering as a problem-solving paradigm whereas design in RtD and PD is not as easily defined (Herriott, 2019). In line with this, for the RtD and PD part of this work, the teach-learn capability of the ML model, is viewed as a design material to be explored concerning its limitations and opportunities. The design experiments are therefore not denoted as prototypes since the connotation of this term point towards a problem-solving process with a product as the goal of the process. In this work the goal for the RtD and PD part is, instead, to better understand the problem domain and refine the research questions based on this understanding. As mentioned before, DSRM aims towards balancing positivism and interpretivism whereas the positivistic stance is represented by DS and the interpretivistic stance is represented by behavioural science. The meaning of design in DS and DSRM is then closer tied to natural science, in contrast to RtD and PD where the word design has a wider connotation. To use Rittel and Webber (1973)'s vocabulary, DS is more targeted towards solving 'tame' problems than design practices like RTD and PD. Consequently, the answer to RQ1 and RQ3 is more in the realms of DSRM and RQ2 relies more on PD and RTD.

\subsection{Research through design}

Research through Design (RtD) (P. Stappers \& Giaccardi, 2017) is an attempt to unite research and design, the two are often regarded as separate endeavours but areas, as, Interaction Design (IxD) has argued for that design and research are inseparable.

The designing act of creating prototypes is in itself a potential generator of knowledge (if only its insights do not disappear into the prototype, but are fed back into the disciplinary and 
cross-disciplinary platforms that can fit these insights into the growth of theory) (P. J. Stappers, 2007).

Central, and one of the challenges with RtD, is the part in the parenthesis, that the researcher has to, either during the process or sometime later, be able to lift out and relate the insights to existing theory. The open-ended research approach of RtD is criticised for the lack of scientific rigour, for example, by Zimmerman et al. (2010) 'As in any mature field of research, there is a need for critical analysis of theoretical outcomes through serious theoretical analysis and criticism'. The view of Gaver (2012) is instead to emphasise this openness as a strength and argue against the natural science-based view on research implying that research has the goal of finding one theory through the process of falsification.

Focus in RtD is on creating and exploring one of many possible futures as an alternative to inductively based research that has a strong focus on the present and the past. One important consequence is that an RtD approach, a priori, opens up for considering ethical and societal implications of the design. In less open-ended approaches ethical and societal implications can initially be left out since they cannot be falsified in a simple manner. In RtD there is no expectation that results have to be exactly replicable, instead research results have to be presented in a form that makes them trustworthy and generative, the results then, instead, tend to be provisional, contingent and aspirational (Gaver, 2012). To handle this openness of RtD, documentation of the process has to be tailored to the problem at hand so it can be used in retrospective. This is often done in the form of design diaries that can be analysed during and after the process. These design diaries are also useful in the PD process that follows and to discuss future usage opportunities, in this work this especially relates to RQ2: 'What new kinds of ML systems can be built based on a HIC-ML approach?'.

The overarching initial goal of the project was, in line with this, not to create a superior commuter app. The goal was instead defined in the following way: a commuter should be able to teach an ML model arbitrary commute patterns given a limited set of features (location, time, day, activity). This goal positions the commuter in command, in relation to the knowledge transfer process, and they can subjectively select the journeys they intend to transfer to the model. The commuter can assess the transferred knowledge in context and in real-time via the journeys the app promotes.

The design process was primarily documented using a Jupyter note- 
book on GitHub, a website and a design diary (See Section 2.2). The findings form a base for the PD study that follows and underpins, by using the DSRM methodology, the final formulation of an abstract model and guidelines for HIC-ML that answers to RQ1.

Some examples of findings from the RtD part of the project, that potentially are generative for a HIC-ML implementation, is an increased focus on the relation between the part of the reality modelled by the commuting app and the reality as a domain expert sees it, different strategies in teaching and that methods involving, for example, personas (Nielsen, 2013) can be useful when designing HIC-ML systems. Personas are here used not instead of involving users, they are used early in the process to generate training and validation data and evaluate teach-learn capabilities of the ML model in a controlled manner. Results from this phase can be found in Paper 1, Paper 2, Section 5.1 and Section 5.2.

\subsection{Participatory design}

To understand the commuter app in relation to the use context and reframe the research questions my perspective is not sufficient, instead data collection methods inspired by anthropology were used. The focus was to explore the teach-learn capabilities of a HIC-ML implementation in a real-world setting for a group of users over some time. A special focus was on discussing different command strategies and evaluate teaching an artefact subjectively selected knowledge. For the data collection, interviews, focus groups and workshops during the usage was used in parallel with background data collection as a type of ground truth related to a specific user's interaction with the app and their travel patterns. The collected data was then discussed and compared with the user's experience of using the commuting app. Commuting as a domain has the advantage that it is wellknown in combination with that the personal data collected is not from any, from a privacy and security perspective, protected data types as defined in GDPR (Otto, 2018). Therefor discussions with me, as a researcher, and between the participant could take place openly.

Co-design is a methodology related to PD that also has a strong user focus and could potentially give insights about the research interests in focus. A difference between these methodologies is the emphasis co-design puts on that users take the role of designers. Since the goal for the work is towards involving users in a mutual learning process, PD seemed like a better choice. 
A PD study is often done as part of an ongoing design process and the design experiment can be seen as a boundary object that functions as a conversational piece that traverses different knowledge backgrounds among study participants. This goes well in line with the intention to involve a group of users in discussions centred on the design experiment at hand to gather insights, both from a concrete commuting standpoint and from a more general level related to HIC-ML. Examples of insights from the study were, for example, that commuters, in this setting, care less about class probabilities concerning predicted journeys they care more about that the model generalises the journey predictions in a, for them, logical manner. The discussions also gave insights on how the user interface could be designed for better explicability (Paper 1 and 2).

Participatory Design has to a large extent Scandinavian roots as a workplace democracy movement. PD aimed initially to involve users of the technology in the transformation of the workplaces. As Winogard and Flores framed the approach 'We encounter the deep question of design when we recognize that in designing tools we design ways of being' (Winograd et al., 1986, p xi). PD acknowledges that designing is taking a stand and it is about designing futures for actual people (Robertson et al., 2012, p. 5). PD has since developed so it today reflects a society that is immersed in digital technology. There are then, for this work, parallels with the profound changes that the introduction of digital technology had to the workplaces 50 years ago with the transformations that we see coming when AI/ML is immersed into our society. The transformation calls for democratic participation and ethical considerations to steer the development in a direction that we want instead of developing the technology we can (HLEG, 2019; Brundage et al., 2020).

For the PD phase, users that could generate discussions both concerning HIC-ML in general but also understand and discuss the implementation of a HIC-ML paradigm in a concrete application was recruited. The users selected had to be daily commuters, with that follows expertise in their commute patterns. Initially, in the mutual learning process, that is central to $\mathrm{PD}$, the participants were introduced to the HIC-ML paradigm so we together during the evaluation could understand and reflect over implications on a more general level. The users selected for the study had different professional backgrounds covering: graphic design, interaction design, user experience design, programming and agile development.

By selecting users from CS-related fields, the participants were accus- 
tomed to discuss, criticise and evaluate design experiments. Since the design experiment discussed was high-fidelity the participants had to be able to see through the polished surface and discuss the implementation from different perspectives and to treat it as a boundary object (Star, 2010). In this limited study, the judgement was made that involving ordinary commuters with no CS relation would focus too much on the limitations of the product. By involving researchers from social sciences would probably not add much since for the concrete implementation of HIC-ML, in this case, there is not much to discuss from a societal and ethical standpoint. However, this decision has implications on the results and it is important to keep these delimitations in mind when conclusions are drawn.

The main form of analysis was made through breaking down recorded material using content analysis (Graneheim \& Lundman, 2004), into codes originating from the machine teaching vocabulary used by Simard et al. (Simard et al., 2017). A more detailed plan can be found at the GitHub repository ${ }^{13}$. The results from this part of the study can be found in Paper 1 and Paper 2.

\subsection{Design science research methodology}

Design Science Research Methodology (DSRM) aims at, as initially discussed, balance positivism and interpretivism in a rigorous methodology through a series of activities: Problem identification, define objectives, design and develop demonstration, evaluation and communication. As discussed above, concretising the results and frame the results from a positivistic perspective opens up for more practical uses of the HIC-ML approach that fits the more 'tamed' problem faced after the RtD and PD parts of the project (Rittel \& Webber, 1973).

IT artefacts are, in DSRM, broadly defined as constructs (vocabulary and symbols), models (abstractions and representations), methods (algorithms and practices), and instantiations (implemented and prototype systems) (Hevner et al., 2004). DSRM, therefore, is well suited to answer RQ1 and RQ3 since they can, more easily than RQ2, be framed via a problem identification. In the case of RQ1, it is in this work answered by defining an abstract model and guidelines, for HIC-ML that answers to RQ1: How would a HIC-ML approach alter the ML system design process?. In the case of RQ3, the outcome of the process is abstractions and vocabularies useful for designing a HIC-ML system when the aim to produce causal

${ }^{13}$ https://github.com/k3larra/commuter 
explications. This then answers RQ3: How can a HIC-ML approach make use of human capabilities combined with ML capabilities to construct causal explanations?

\subsubsection{DSRM and RQ1}

DSRM focuses on defining and solving problems using activities that involve demonstrating an artefact and evaluating it. The artefact developed, in relation to RQ1 using DSRM, is then, not the design experiments used previously as boundary objects, instead, the focus is on developing an abstract model and guidelines. The design experiments (See section 2) are then instantiations and part of demonstrating the viability of the abstract model and the guidelines.

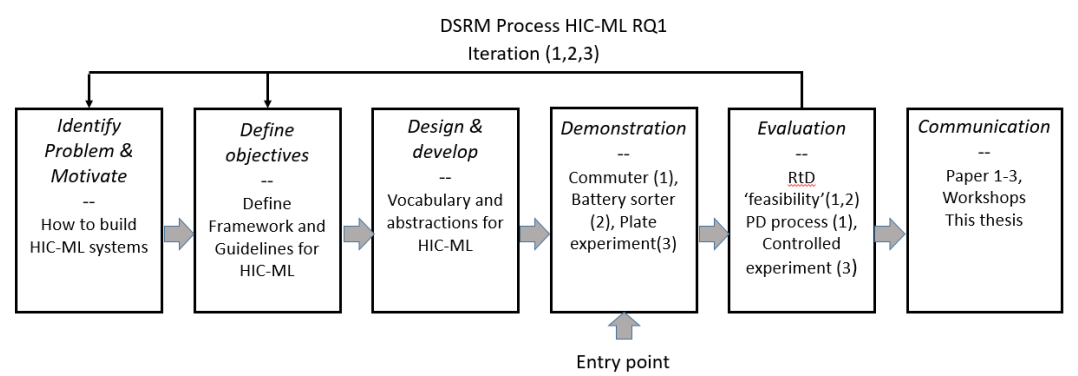

Figure 7: The DSRM activities adapted to the usage in this thesis to answer RQ1: How would a HIC-ML approach alter the ML system design process?. Numbers 1-3 indicate the design experiments, $1=$ Commuter, 2 = Battery sorter and $3=$ Plate design experiment.

Evaluating the abstract model and the guidelines are conducted using the design experiments in an iterative process. Following the DSRM process as Peffers et al. (2007) outlines it, the starting point can be chosen freely, and in this case, the process was entered with the experiences from the commuter app as the first demonstration of a HIC-ML system. Both the HIC-ML abstract model and the guidelines were developed iteratively using the three design experiments to define the guidelines. The iterations ended when the abstract model and the guidelines matched the three design experiments. The activities and the processes are outlined in Figure 7. The model evaluation concerning the RtD design experiments (commuter app and the battery sorter) are denoted 'feasibility' studies. This since they as instantiations of HIC-ML evaluates the feasibility of the approach, but at the same time help to inform and define the HIC-ML abstract model and the guidelines. Similarly, the PD process for the commuter app both 
evaluates, informs and defines. This then together forms the answer to RQ1: How would a HIC-ML approach alter the ML system design process?

\subsubsection{DSRM and RQ3}

This research question is answered in the form of a vocabulary and abstractions developed to be useful when designing a HIC-ML system that has as its goal to use human capabilities combined with ML capabilities to construct explanations. Here, the focus is on scientific explanations as a rigid form of explanations useful as a blueprint for explanations in a HIC-ML context as discussed in Section 3. Theories from social science and philosophy were used to find abstractions suitable to discuss how explications in the form of causal attributions produced by the ML system can become causal explanations in the hands of domain experts. Finally, an abstraction in the form of a trust quadrant is developed and used to discuss how trustworthiness relates the the examples the ML model is exposed to, both those examples that are part of the problem formulation but also those examples not covered by the problem formulation. The abstractions and the vocabulary is evaluated using the design experiments. The DSRM process that is used with RQ3 can be found in Figure 8.

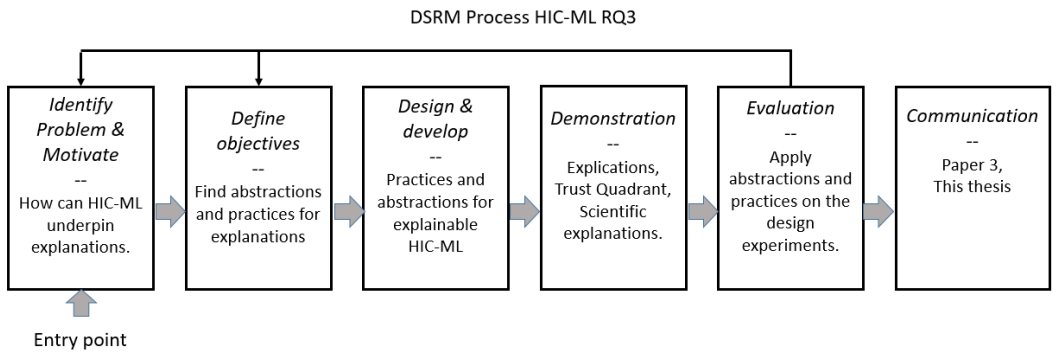

Figure 8: The DSRM activities adapted to the use in this thesis to answer RQ3: How can a HIC-ML approach make use of human capabilities combined with ML capabilities to construct causal explanations?

\subsection{Concluding methodological remark}

To answer the research questions a mix of methodologies that rely on different epistemic stances has been used in the knowledge-producing process for this thesis. Answers related to RQ1 is more inward-looking based on the craft of creating information systems and therefore the goal and utility-focused methodology DSRM fits well. The work done using the RtD and PD part of the work presented matches RQ2 since that question 
aims towards knowledge production that is explorative but still generalisable and generative. The contributions can therefore point toward future application areas. Then DSRM is used to underpin the answers to RQ3 since that question, as RQ1, is more about concrete results. To RQ1 and RQ3 the instantiations, to use the DSRM terminology can be seen as design experiments whereas they are, in RQ2, treated as boundary objects, to use an RtD/PD terminology. 



\section{CONTRIBUTIONS}

The contributions are organised in relation to the research questions presented initially in Section 1. As earlier discussed RQ1 uses a DSRM approach to produce a vocabulary, an abstract model over the usage of an HIC-ML system and propose guidelines helpful when designing and building HIC-ML systems. RQ2 points towards possible target domains for HIC-ML. The results are underpinned by RtD and PD as research methodologies and consequently present generative results and explores possible futures using the three design experiments. For RQ3 DSRM is again used to establish abstractions and vocabulary to design for explicable HIC-ML. The target audience for the contribution is a design team responsible for developing HIC-ML products.

\subsection{RQ1: How would a HIC-ML approach alter the ML system design process?}

An answer to this research question is formulated as an abstract model and guidelines as an alternative to a view on traditional ML that delivers a, to HIC-ML, comparably static product when it comes to training and deployment (See Section 3.2). The answer was constructed over several iterations on DSRM activities (visualised in Figure 7). Since the commuter is the only design experiment used over time it is hard to propose guidelines that concern how interaction over time relates to the mental model a user internalise (See Section 3.7). The commuter is also the only design experiment built with high fidelity and guidelines connected to UX and IxD are, due to the limited empirical material available, hard to concretise. That design experiment focused mostly on the teach-learn capabilities and less on the UX and IxD design, insights related to this are instead illuminated in the discussion section (Section 6.3).

The abstract model for HIC-ML usage is initially presented, on this follows the proposed guidelines. The abstract model and the guidelines combine knowledge based on theories and knowledge created using the design experiments. The relation between the proposed guideline, the theory and the prototypes is clarified after each guideline. 


\subsubsection{Abstract model for HIC-ML usage}

Below an overview of the abstract model in Figure 9 is presented. The terms and vocabulary used are not new to machine learning instead they are used to connect the abstract model with the guidelines.

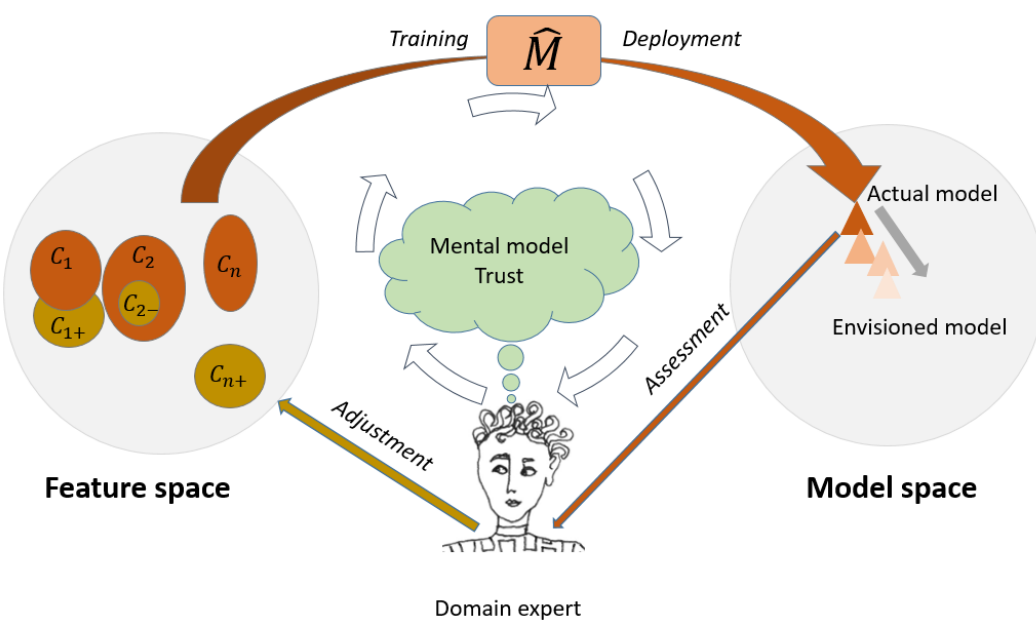

Figure 9: Abstract model outlining the HIC-ML approach.

Domain expert(s) iteratively selects and deselects training data in the Feature space (Guideline 5.1.2), initiate ML model training, uses the system and assess the learned knowledge. The command aspect is represented by the Adjustments' arrow (Guideline 5.1.4), adjustments are based on the domain expert's teaching goal, domain expertise and Assessment (Guideline 5.1.5) of the knowledge already transferred to the ML model. Over time and usage, the domain expert can build a Mental model (Section 6.3) that incorporates dynamics of the HIC-ML system, important for the creation of a human-machine relation needed to, over time, gain trust in the system. A mental model can include didactic understanding related to how to best teach the ML model ('machine pedagogy'). The mental model the domain expert creates internally, during usage, includes an understanding of the limitations of the system e.g. the Problem formulation (Section 6.3). The Model space (Guideline 5.1.3) (hypothesis space) represent all possible hypothesis that can be transferred to the ML model. By adding or removing training data the ML model moves in this space so it in an ideal case at some instance of time matches the knowledge the domain expert intends to transfer. This goal is in Figure 9 denoted Envisioned model. 
Clarification in relation to HIC-ML target domain. The ability for a domain expert to select a target domain is limited by the part of reality exposed to the ML model via the features, either raw as images, sound and text or engineered as location, in combination with the selected ML method. To exemplify, the commuter design experiment target individual commute patterns that use the features: time, date location and activity to predict an upcoming journey. The conceptual battery sorter's generalisation is bound by the physical machine it is connected to. The conceptual plate design experiment is more general and its capabilities in classifying objects are limited by the quality and availability of training data, knowledge priors in a transferred model, compute power and adaptability built into the UX design, especially when it comes to assessing promoted decisions.

\subsubsection{Design guideline: Feature space}

The feature space delimits the part of the domain available for the ML model to learn from. Guidelines presented below are separated in relation to engineered features, like those used by the commuter app, and raw input data (images, sound, text) (See Section 3.4). Settings were engineered features and raw input data are combined can also exist.

Design guidelines for engineered features:

- Select features so they are part of the target domain's ontology they can then be understood by the domain experts and simplify training data adjustment.

- Avoid feature engineering in conflict with domain conventions, if this cannot be avoided communicate them to the domain expert.

- Give domain experts opportunities to add or remove features since this can simplify the ML model, reduce training time and make it easier to produce explicable decisions.

Clarifications and rationale. Related to feature engineering, if the commuter app average distances to steps of 200 meters, due to some technical limitations, a commuter would have a hard time understanding why the app cannot distinguish between two close stations. The app has to be designed so this limitation is communicated. Additional features like weather and calendar data were proposed during the evaluation of the commuter, these would if implemented add new dimensions to the feature space. Features in the app could be ignored so for example journeys could 
be predicted based only on time of day which simplifies understanding (See Figure 10). Additional discussions can be found in Paper 1.
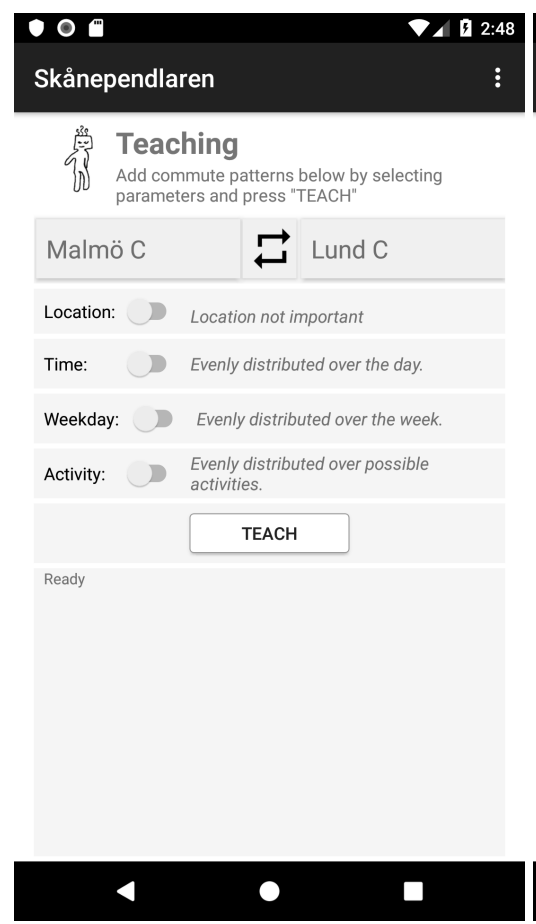
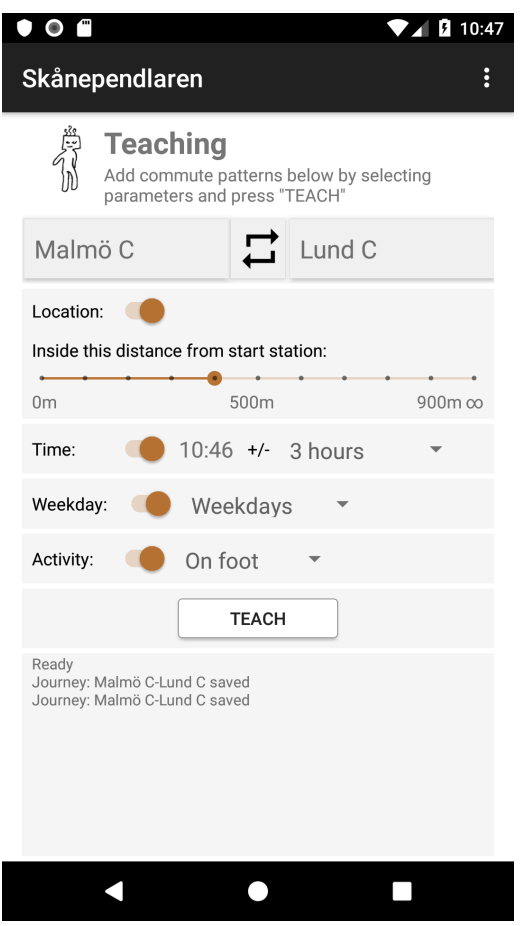

Figure 10: Figure: Teaching interface for the commuter app. To the left, a journey from Malmö C to Lund $\mathrm{C}$ is taught as a default journey independent of any feature, to the right a more specific journey from Malmö $\mathrm{C}$ to Lund $\mathrm{C}$ is taught.

Design guidelines raw input data:

- Metadata, for example, resolution, colour depth, etc. should be communicated to the domain expert. Selection of metadata that needs to be exposed to the domain expert has to be judged by the design team in relation to the intended use context.

- The domain expert should be able to deselect and select data augmentation settings for raw input data to increase context fit. The design team has to judge which data augmentation settings that need and can be exposed to the domain expert.

Clarifications and rationale. For the battery sorter and the plate design experiment, raw input data in the form of images were used. From an ML model training performance perspective, fewer and low-resolution images 
are preferred since training time grows fast with many high-resolution images. For the plate design experiment the images needed to be at least $480 x 480$ px for the neural network to be able to identify a plate. Consequently, if resolution settings are not available or communicated to the domain expert it is hard to understand why the ML model does not learn. By presenting the images used for training for the domain expert they could review if the image is detailed enough. In this type of training, data augmentation is often used to limit the amount of training data needed. Data augmentation like resize, skew, colourise and flip has to be used with care. This type of augmentations creates more training data and helps the neural network to reach higher accuracy on the training data but can distort the ML model's ability to represent reality. For example, if flip (mirror the image on a central axis) or colourise (change colours) are used for plate images these images will teach the ML model about a plate that does not exist in reality, something a domain expert can understand more easily than an ML expert.

\subsubsection{Design guideline: Model space}

The model space or hypothesis space represents all possible model instances or hypotheses that can be learned by the ML model.

- Focus, when designing the system, on how the ML model generalises decisions in relation to the feature space.

- Give, when designing the system, the domain expert the power to delimit the ML Model's generalisation based on, for example, class probability.

Clarifications and rationale. For the commuting app, users found it logical that training a journey on a Monday morning generalise to mornings on all workdays. In Paper 2 some examples of how generalisation can be visualised for a commuter are presented. Accuracy can be used to limit generalisation or indicate that an example should be assessed. To use the plate example low-class probability can indicate either that the image is of low-quality or that the ML model has not been trained to identify it.

\subsubsection{Design guideline: Training data adjustment}

The domain experts need to be able to, during usage or prior to usage, develop a didactic understanding related to the ML model and thus be able to select teaching strategies. This didactic understanding is seen as part 
of the mental model discussed in Section 6.3. Teaching strategies can be: intuitive and/or possible to understand and learn for the domain expert. Examples of teaching strategies that the domain expert can use that can be communicated to the domain expert:

- Use typical or representative examples for a label.

- Use border examples that are as similar as possible but with different labels.

The best training strategy is dependent on previous training, this is pronounced in neural networks (Bengio et al., 2009). An initial strategy to teach using representative examples followed by border and counterexamples to adjust the knowledge can be one useful strategy (Lindvall et al., 2018; Ramos et al., 2020).

A HIC-ML system has often no initial training data or labels and that information has to be added when the teaching is initiated. This can be handled using one or a combination of ML approaches complemented with matching UX design.

- Collect and label new data from the intended deployment context (discussed in Paper 1). This is an approach useful when none of the approaches below can be used, a drawback is that it can take time to collect enough training data during usage.

- Import an existing labelled/unlabelled training set from a related domain and label, add, modify or tweak this data. This is useful when the HIC-ML system uses engineered features and similar public labelled datasets exists that can be translated to match the engineered features.

- Use transfer learning, import a pretrained model and retrain it to target the intended domain (as in the plate and battery experiment). This approach is useful to minimise the need to collect domain-dependent training data since more general representations are already learned by the neural network.

- Label sub-spaces in the feature space and generate examples representing these spaces (as in the commuter example). This approach is useful when engineered features are used and a domain typical examples can be inferred from the intended target domain. 
Clarifications and rationale. For the commuter app (Figure 10) a teaching interface made it possible to select a sub-space in the features space and generate labelled data inside that space (this is discussed in more detail in Paper 2). For the battery sorter and the plate design experiment, no teaching interface was constructed. To give some indications of the amount of training data needed: the plate design experiment used around 40 images of each plate with good quality to reach high accuracy and the battery sorter used 3 images of each battery to reach an acceptable classification result. The first two guidelines are derived from ML cold start strategies, the last two proposed guideline are part of the design experiments referenced in this work.

\subsubsection{Design guideline: Assessment}

These guidelines used in conjunction with the notion of explicability (See Section 3.5) and the trust quadrant (See Figure 5) can be used to assess the decisions. The guidelines are central for the domain expert's prospect to build a useful mental model of the system.

- Design the system so a domain expert can teach and assess the system in arbitrary short cycles so they can build a mental model of the HIC-ML system's limitations and capabilities.

- Use local explications to build global human understanding and express these explications using the target domain's ontology.

- Communicate to the domain expert the segment of the domain wherein the HIC-ML system can be trusted (See Section 5.3).

Clarifications and rationale. The guidelines refer to the human capability to over time build a mental model of complex systems, based on local knowledge, which in turn makes it possible to understand how the model's knowledge relate to the target domain. Possible explications are limited by the target domain but also the method selected by the design team to communicate explications, for example, saliency maps or text-based decision sets (Selvaraju et al., 2020; Lakkaraju et al., 2016).

Explications for the design experiments referenced in this work takes different forms:

- Direct contextual comparison, as in the commuter example. This implies that the commuter compares their expected journey with the predicted journey. 
- Physically analysing the result compared to metrics decided by domain experts, as in the battery sorting example (using a confusion matrix as visualised in Figure 2).

- Visual explications that the domain expert can relate and compare with their understanding. This is discussed in Paper 3 related to the plate design experiment.

Neural networks used in this work cannot create causal explanations tailored so a user can understand them, instead, they find correlations that can be presented as causal attributions connecting internal representations with decisions. The system can then only produce causal attributions that imply, for example for the commuter, given a place and time a specific journey is proposed, instead of an explanation that gives information on why the commuter intend to take the journey (this is discussed in more detail in Paper 2).

For the domain expert using the system, it is important to understand, not only when the ML model's decisions can be trusted but also when they cannot be trusted. If and only if an example exposed to the model belongs to Known Unknowns the model can be expected produce trustworthy decisions and explications. It is then central for the domain expert to understand that decisions concerning Unknown Knowns and Unknown Unknowns can erroneously be made with high confidence by the ML model and consequently present an explication tailored for a Known Unknown. If the domain expert is aware of this the explications the HIC-ML system produces can, even-though not being trustworthy, contain valuable information useful concerning Unknown Knowns and Unknown Unknowns. For example, that one plate is very similar to one in the collection and therefore can indicate that it is painted by the same artist.

\subsubsection{Summary}

The proposed guidelines focus on four areas in the abstract HIC-ML model (See Figure 9): feature space, model space, training data adjustment and assessing promoted decisions. Guidelines concerning feature space and model space address the part of reality available to the ML model and its ability to learn from these features. Design guidelines on adjusting training data and assessing learned knowledge are closely connected and together they represent the dynamic part of the abstract model for HIC-ML. 


\subsection{RQ2: What new kinds of ML systems can be built based on a HIC-ML approach?}

The domain a specific HIC-ML system can target depends on the overall design. At the heart lies the ML model's capability to be trained on and promote decisions for a domain. Assessing and understanding these decisions follows as a central demand together with the capability to train the model independent of IT professionals. User interface, interaction and user experience design then delimit the versatility of a specific HIC-ML system.

To answer RQ2, the application domains for the three design experiments were used. These domains indicate how results from the RtD process can be generalised. The discussions then, related to the other research questions, becomes more speculative since the results build on knowledge gathered through RtD processes. Domains expanding the contexts the design experiments target are:

- Intelligent Personal Assistant, Assistive Technology, Personal Informatics. Represented by the commuting app.

- Sorting physical items: for example: Coins, collections of similar artefacts, weed out, etc. Represented by the battery sorting design experiment.

- Systems that enhance human cognitive capabilities. Represented by the plate design experiment.

These target domains are not new to ML, instead, the HIC-ML approach adds a design layer that transforms them, from a design and usage perspective, by moving the teach-learn capabilities of the ML system towards the users.

\subsubsection{Commuter App: Design experiment}

This design experiment is described in more detail in Section 2.1 and Paper 1-2. The app shows that an individual ML model can be trained and function as Assistive Technology and be used to support decisions related to Activities in Daily Living (ADL) (Lawton \& Brody, 1969). This can include, for example, medication reminders, finding the way to specific stores, etc. The strength relates primarily to privacy since the trained model is not shared, that it is personal and training builds on specific needs for a user instead of similarities with other users. Sharing model knowledge 
through for example federated learning (Brundage et al., 2020) can then take place with less privacy concern than in ML systems where data and ML models for different users are not separated.

From a technological perspective, using container technology in the cloud for training combined with encrypting feature values at the source and local decryption of decisions can make it very challenging to break the privacy. Most systems targeting similar areas like Alexa, Cortana and Siri can act as intelligent personal assistants, but data in these systems are shared with the tech giants behind the products, which is not ideal from a privacy perspective.

An example of a simple HIC-ML system could be setting up a geofence together with an elderly person that sometimes gets lost. This can then be done together with the end-user by walking the perimeter and deciding whom to send a message to if the perimeter is broken. These kinds of systems can then be trained on a combination of features like time, date, location, weather, etc., without the need for explicable decisions since the functionality can be verified in context similar to how the commuter app works. A possibility to get an overview of the knowledge transferred to the system will be important if the knowledge is complex or to delete obsolete knowledge.

The discussions here do not bring forward a type of assistants that try to completely understand human modes, intentions or plans as proposed for example by Cohen et al. (2016). Instead, the intention in my work is more low-key and with the objective to subjectively create meaning and value for the domain experts and users. HIC-ML in this case targets a personal knowledge domain. This does not rule out that the teaching and the usage can be separated between users, perhaps with different privilege levels, as in the geofence example above. How governance principles are implemented in cases like this is an open question.

\subsubsection{Sorting items: Battery: Conceptual}

This design experiment is described in more detail in Section 2.2. The technology used is similar to the hybrid systems described below, but in this case, some type of sensors and actuators are connected to the system. Usage envisioned are for example Weed-Killing Robots ${ }^{14}$ that can be trained by farmers or more general sorters that can be trained to target

\footnotetext{
${ }^{14}$ https://interestingengineering.com/how-weed-killing-robots-are-destroying-agrochemicalgiants
} 
a local domain ${ }^{15}$.

In these examples, the HIC-ML system replaces tedious work or are faster or stronger than humans. In the case of weed out, manual methods could replace or minimise the use of pesticides. Assessment of the model's capabilities can then be physical as in the battery sorter example.

\subsubsection{Hybrid systems: Plate design experiment}

Being able to identify, remember and distinguish details in physical objects is demonstrated by the plate design experiment. Existing techniques (Lundberg \& Lee, 2017; Ribeiro et al., 2016) can lift out salient details related to the classification, details that can be used to build trust in the system. Our design experiment also showed that by using transfer learning, collecting enough data to classify an item is a feasible task that requires a reasonable amount of training data.

A HIC-ML system with the qualities demonstrated, could be used by biologists too, in context, train a model to distinguish individual insects or mammals from the same species. This could, for example, be an alternative to ringing birds or give researchers a possibility to study individual bird behaviour with a granularity that is not possible today (Ferreira et al., 2020).

A related area that has many characteristics in common is radiology and classifying tissues in images (Lindvall et al., 2018). Here a HIC-ML approach can open up for knowledge sharing between professionals so trained ML-models can be downloaded and used to make initial sorting of radiology images when resources are scarce. Governance principles in the HIC-ML system have to be developed so domain experts can add their expertise to the system and explications can be contested and discussed.

\subsubsection{Summary}

Moving agency in ML towards end users opens up for for new design perspectives, as described above. This shift towards a target domain will then be demanding from a design perspective since HIC-ML adds teach-learn capabilities to an ML system and these capabilities needs to be adapted. An HIC-ML approach will imply a specialisation and differentiation, perhaps not so much with respect to ML methods, but more from a user and usage perspective.

\footnotetext{
${ }^{15} \mathrm{https}: / /$ www.youtube.com/watch? feature=player_embedded \&v=OugqnVO7WiU
} 


\subsection{RQ3: How can a HIC-ML approach make use of hu- man capabilities combined with ML capabilities to construct causal explanations?}

To answer this research question abstractions and practises are created, they are produced to be useful for a design team when the goal is to create explicable HIC-ML systems. The design goal is then systems that support a domain expert's understanding and consequently their ability to explain promoted decisions. The use of a domain expert's capabilities when an explanation is created is central to answer this research question. The practices and abstractions adapted for HIC-ML are first presented below. They are then applied to the design experiments to clarify their applicability. Finally, conclusions are presented as a condensed answer to the research question.

\subsubsection{Abstractions and practices}

To build trust in the HIC-ML system the goal is to create causal explanations resulting from an interaction between the ML explicator and the human explainee, the process follows on the explainee's interest in understanding why a specific decision was promoted by the HIC-ML system. This process, driven by the explainee, ends when the explanation forms understanding concerning a promoted decision. The explainee's goal is then to transform, using domain knowledge, the causal attributions the ML model can be expected to produce into a causal explanation.

\section{Trust Quadrant}

\begin{tabular}{|c|c|c|}
\hline & Knowns & Unknowns \\
\hline Known & $\begin{array}{l}\text { Trustworthy explications } \\
\text { Confident decisions } \\
\text { (Known Knowns) }\end{array}$ & $\begin{array}{l}\text { Potentially useful explications } \\
\text { Non-confident decisions } \\
\text { (Known Unknowns) }\end{array}$ \\
\hline Unknown & $\begin{array}{c}\text { Potentially useful explications } \\
\text { Arbitrary confidence level } \\
\text { (Unknown Knowns) }\end{array}$ & $\begin{array}{l}\text { Explications that can be } \\
\text { misleading or wrong } \\
\text { Arbitrary confidence level } \\
\text { (Unknown Unknowns) }\end{array}$ \\
\hline
\end{tabular}

Figure 11: The top row include all examples the ML model is trained to promote decisions for and consequently explications concerns examples known to the model. In the bottom row, all examples are unknown to the model and the ML model cannot produce correct decisions and explications for these decisions can consequently not be trusted in relation to the target domain. 
Scientific explanations and a common form of human explanations are both, as discussed in Paper 3 and Section 3.5, initiated by a why-question. Overton (2012)'s structure for scientific explanations is chosen since it can be used to understand the type of knowledge a HIC-ML system can learn.

To discuss the validity of explanations the knowledge quadrant (See section 3.3) was expanded with notions related to trust (See Figure 11). The resulting trust quadrant can then, together with Overton's model, be used to discuss the validity and scope of the causal attributions the ML model can produce and present. The trust quadrant's focus is to clarify the knowledge gap between the model, the domain expert and the reality.

A HIC-ML system can produce explications for the different categories in the trust quadrant as presented below:

- Known Knowns: Trustworthy explications can be created since they build on labelled examples representing a ground truth. Trustworthiness for explications related to the other categories build on these explications.

- Known Unkowns: If the confidence level is high explications are likely more trustworthy. This since the confidence level indicates that the example belongs to the target domain.

- Unknown Knowns: Explications can give the domain expert more insight. If an example from this category is classified with a high confidence level it is similar, in some respect, to a Known Known.

- Unknown Unknowns: The domain expert and the ML expert is unaware of the existence of these examples that still belong to the domain. Explications can therefor be misleading or wrong. Examples from this category can hide in any other category. This is the category where black swans and white ravens linger; and also, an opportunity to falsify the theory or presumptions the ML model builds on.

\subsubsection{Applied on the commuter design experiment}

This design experiment augment human abilities in the sense that a personal commute pattern can be transferred to an app that in real-time can predict upcoming journeys based on the commuters context (See Section 2.1). The app provides an explication that connects contextual data with a journey but leaves to the commuter to associate the journey with a 
purpose. For a user that knows their commute pattern the commuter app only saves time by automating one part of the functionality of the app. If the app functions as assistive technology for a person with some type of cognitive impairment the app can act as decision support. In both cases explaining why they travel from A to B is in the hands of the commuter, the app adds evidence for an explanation by presenting an explication that connects the commuters context with a journey. The commuter can then, over time, build a mental model of how the performed training relates to the predicted journeys (Paper 1).

The core relation, using Overton's model of scientific explanations, to be explicated is between a kind (a journey) and data (location, time, day, activity). The HIC-ML system does not give any information on alternative journeys predicted with lower class probability. This implies that there are no contrasting kinds/journeys that potentially can be useful for the commuter to better understand the ML model and consequently improve the mental model. The categories theory and model in Overton (2012)'s model are not a part of this HIC-ML implementation. By adding the model category to the app, journeys could be grouped related to concepts, for example to and from work, etc., concepts that can function as further evidence for a decision and be added to the explication.

If a commuter remembers the training they performed they will know which journeys the app can be expected to predict (Known Unknowns). If an expected, a Known Unknown, is not predicted correctly more training data can be added that relates to this journey so it can be predicted correctly. A commuter also knows that the app cannot predict a journey not trained (Unknown Knowns) and that they can train the ML model to include this new entity/journey as part of their commute pattern/domain. The commuter app cannot be exposed to the Unknown Unknowns category since all possible departures are, in theory at least, known or searchable in the app.

\subsubsection{Applied on the the battery sorter}

In this design experiment, the capabilities of the HIC-ML system augment human abilities and the outcome can consequently be evaluated using human cognition (See Section 2.2). The design experiment provides an explication that connects a sorting outcome with either $\mathrm{NiCd}$ or alkaline. For a recycling expert, the envisioned system makes sorting more effective.

The core relation to be explicated is between the data and the two 
kinds of batteries: alkaline and $\mathrm{NiCd}$. The data used for classification is in the form of images picturing individual batteries. The core relationship between the kinds (alkaline, $\mathrm{NiCd}$ ) and the data (images) consists of subkinds (the different brands/articles) and the entities (individual batteries) representing these sub-kinds. The battery articles are then represented by data in the form of images used as training data. By inspecting the sorting outcome a recycling expert can, for example, suspect that a missclassification can build on that battery design for a specific alkaline battery article is very similar to a $\mathrm{NiCd}$ battery. In this design experiment, there is no possibility to inspect the causal attributions the ML model base a promoted decision on. Trust can only be built by evaluating the result, either locally by inspecting one decision or globally by inspecting many decisions. In this HIC-ML system knowledge concerning theories related to recycling and how that knowledge is implemented via a model into kinds is unknown to the HIC-ML system. This defines the human domain knowledge needed to complement the ML system's knowledge to create a working HIC-ML system that can augment human abilities.

The target domain can be expected to shift over time with new designs and new types of batteries. The recycling experts have to create a control mechanism to encounter this. This domain shift implies that the model regularly will be exposed to Unknown Knowns, which can be identified via human inspection, and Unknown Unknowns, that can pass human inspection. If the model reaches high accuracy on test data, accuracy can be used to sort batteries in a third category that has to be inspected by a domain expert. This category then contains Known Unknowns (Batteries that are, for example, worn), Unknown Knowns (Battery kinds the ML model is not trained for) and Unknown Unknowns (Battery kinds that are, for example, $\mathrm{NiCd}$ batteries with the similar appearance as an Alkaline battery). But as explained in Section 3.3, high accuracy for a decision can not rule out that the entity classified is an Unknown Known or an Unknown Unknown.

\subsubsection{Applied on the plate design experiment}

In this design experiment the HIC-ML system aims to enhance human cognitive capabilities and the outcome can therefor not be evaluated using human cognition (See Section 2.3). The problem formulation is delimited by a collection of six designer plates. These six plates are classified with $100 \%$ accuracy under the ideal conditions we used for the study. The 
situation we focus on in Paper 3 is a setting where the entities (plates) from a human perspective are very similar. In addition, Unknown Knowns (plates the model has not been trained to identify) are hard for a human to distinguish from a plate that belongs to the collection.

The design experiment produces an explication in the form of several causal attributions by highlighting areas in an image important for a proposed decision. Paper 3 shows that some of these attributions can be used by the domain expert to build causal explanations and consequently over time increased the domain expert's domain knowledge. The work indicates that increased trust in the HIC-ML system builds on that the causal attributions lifted out overlap the domain ontology known by the domain expert.

Paper 3 also shows that causal attributions for this design experiment can use the categories kinds, entities and data in (Overton, 2012)'s model. The core relationship, in this case, is between the entities (individual plates) and the image data. The kinds category consists of only one kind: handpainted Spisa Ribb designer plates. The visual causal attributions give, via the entities and kind category, access to both causal and contrastive attributions. A domain expert can, in addition to these attributions, use the categories theories and models when formulating a causal explanation. The HIC-ML system, can not, as it is trained in our experiment, for example, distinguish hand-painted plates from printed plates. The domain expert needs to understand this type of limitations of the HIC-ML system to trust it.

\subsubsection{Summary}

For a HIC-ML system to underpin a domain expert's ability to produce explanations, the following design approaches are proposed to increase their trust in the explications the ML system produces:

- Explications should be presented so they overlap the target domain's ontology and consequently can be understood by the domain expert.

- The delimitation of the domain the ML model is trained for needs to be obtainable for the domain expert.

Comment to first item: For HIC-ML system that augment human abilities explications can be evaluated by a human that compares input data and the promoted decision (Battery sorter and Commuter design experiments). For systems that enhance human cognitive capabilities explications produced 
by the system are vital information for the domain expert's ability to construct explanations (Plate design experiment). Overton (2012)'s model of scientific explanations is useful to understand to what extent human capabilities are needed to complement the ML system's capabilities.

Comment to second item: The domain expert has to be able to distinguish between explications that relates to examples the model has been trained on and those it has not been trained on, since explications inside and outside of the problem formulation has to be interpreted differently. The trust quadrant (Figure 11) can be helpful when the design team discuss and address this. To be able to trust the HIC-ML system the domain expert needs to know the domain delimitation. The HIC-ML system can then either, in a simple case, rely on that a domain expert remembers past training or take notes. Or, preferably, that that the HIC-ML is designed to facilitate these needs and can present accumulated knowledge for the domain expert in an understandable fashion. 



\section{DISCUSSION}

The three design experiments used to produce the contributions covers only a delimited part of the HIC-ML area. This is understandable and in line with the methodological approach that aims at exploring possible futures. The results presented in Section 5 point towards new design perspectives as well as proposing approaches to HIC-ML product design. The discussion section that follows aims to bring unexplored research areas into focus, generate further research questions and open up for scientific critique related to the HIC-ML perspective.

Initially, conclusions are drawn and discussed concerning the results in Section 5. The discussion is divided into two major strands: Firstly, HICML systems augmenting human abilities without having, from a cognitive perspective, abilities hard to reach for humans. The outcome of a decision proposed by the ML system can then be assessed using human cognition (See Section 6.1). Secondly, systems that outperform humans from some cognitive perspective resulting in a situation where the system has to be able to provide evidence that can be used by the domain expert to explain and understand a decision. The design experiments can in the second case be compared to scientific instruments, like a digital microscope, that transforms and translates details of the reality (See Section 6.2). On that follows a discussion around challenges and opportunities revealed during enquiries into HIC-ML (See Section 6.3). The next section contains a discussion concerning the virtue ethic stance used to approach HIC-ML (See Section 6.4). In the next section limitations in the HIC-ML approach to ML are discussed (See Section 6.5). The chapter ends with a future work section.

\subsection{HIC-ML augmenting human abilities}

HIC-ML that augments, amplifies or enables the domain expert's abilities is represented by two design experiments: the battery sorter and the commuting app. In these experiments, human knowledge is transferred to the HIC-ML system and the system assists in a human endeavour. In Section 5, the battery sorter and the commuter app were differentiated based on that only the sorter can directly act in the physical world using its actuators. 
The augmenting approach implies that decisions can be evaluated by a domain expert that compares input data with the proposed decision.

From a teaching perspective, the learning goal when augmenting human abilities can be well-defined and the domain expert can avoid exposing the model for examples that belongs to Unknown Knowns. The situation resembles more static ML situations, the differences being that the command principle gives the domain expert power to decide use context and over time redefine the problem formulation. Ramos et al. (2020) use Interactive Machine Teaching (IMT) to discuss the problem from a similar perspective and focuses on transferring knowledge to the learner, not only as labels but also as concepts and taxonomies. In that work, there is a ML model performance focus that differs from the focus in this work, which centres on value and meaning creation for the domain expert/user in control and responsible for direction, purpose and ethical considerations concerning the usage. Ramos et al. (2020) consequently brings forward the possibility to share a trained model with other users that can perform a well-defined task as a possible route to transfer knowledge between IMT settings. Depending on the context, sharing trained models in line with IMT for a public or shared knowledge domain is a route that has interesting bearings for HIC-ML.

\subsection{HIC-ML enhancing human capabilities}

This setting shares many characteristics with the setting discussed above that augment human abilities. A central difference is that a domain expert either has to take contextual precautions concerning Unknown Knowns or rely on and trust the explications the HIC-ML system produces. For example, if two plate collectors (See Section 5.3) accidentally mix their collections, an ML model trained only on one collection, cannot rule out the possibility that there is one very similar plate in the other collection that makes it hard to divide the collections. This type of problems cannot be solved by focusing only on class probability the system has to be able to provide useful causal attributions as an explication.

For an real-world problem, there are, as illustrated by the bird identification example (Ferreira et al., 2020), additional challenges concerning Unknown Knowns and Unknown Unknowns that cannot be completely solved using contextual precaution. For example, how can a bird watcher know if it is the same individual bird when a bird return after the winter or during moulting? Evidence for the identification, in that case, has to 
rely more on unique causal attributions (like scars) instead of contrastive attributions (like brighter feather colours).

In these situations, domain expertise combined with explications presented by the HIC-ML system has to be used insightfully to make the system useful. Since HIC-ML is about subjectively create meaning and value the system must be trustworthy and not seductive. As discussed above in Section 5.3 explainability for HIC-ML aims for causal attributions as evidence for an explication to answer an explainee's why question. Since the process of finding an explanation is driven by the human explainee using their domain knowledge, the room for seductive decisions can potentially be restrained compared to a situation where the system produces a non-contestable explanation.

\subsection{Mental model and UX}

In this section, areas that need further research to produce practical guidelines and design patterns are brought forward.

A mental model internalised by the domain expert that encompasses the HIC-ML system's capabilities is a form of human ability that can match challenges connected to trust in HIC-ML (See Section 3.7). For the commuter app, it was relatively simple for commuters to internalise a mental model of the commuting app's capabilities since the teaching needed to transfer a commute pattern was limited and the promoted decisions are evaluated in real-time during usage. The users could refer to this as for example 'I trained on a Monday and this is Wednesday same place and time and therefore it predicts the same journey'.

It is important to take into account that the learning goal can shift and develop based on both shift in the use context as well as a domain expert's shifting usage needs. For example, for the commuting design experiment, shifts can be due to; changes in bus routes as well as shifting commute pattern for the commuter.

The work presented in this thesis indicates, in line with contemporary research, that addressing $\mathrm{UX}$ and $\mathrm{IxD}$ challenges in the field is an open research question (Dove et al., 2017; Dudley \& Kristensson, 2018; Yang, 2018). Challenges from a UX perspective stands out related to the coadaptive process that involves the domain expert and the ML-model, a process that evolves. A focus on participation during design is therefore central, this especially includes involving representative domain experts. Here traditional methods, for example, Wizard-of-Oz, scenarios or per- 
sonas can be used (Nielsen, 2013; Dahlbäck et al., 1993; Gudjonsdottir \& Lindquist, 2008). These methods are especially useful in the earlier parts of the design process since they can be used to understand how the teachlearn capabilities of ML can be utilised in key situations by emulating proxy humans and proxy tasks. This is then an opportunity to create fictive but realistic situations and experiments in a controllable and reproducible fashion. Especially the reproducibility is useful to optimise ML model performance and evaluate ML model generalisation. Personas and scenarios were used in this way to develop the commuter app and to make sure that the app could function well in key situations for envisioned user types. For the commuter, this controllable and reproducible evaluation phase lifted the project to a level where evaluation with real humans and real tasks became meaningful (See Paper 1).

The relation between a human and a well-functioning HIC-ML system will, at some stage, reach a point where the transferred knowledge approaches or even matches the domain expert's learning goal, consequently, the usage can call for different interaction patterns during the system's lifetime. For example, in the plate example, an initial intensive teaching period, based on an MT approach, can be followed by a more sparse assessment period, based on an IML approach centred on, statistics on promoted decisions, adjusting metadata related to the plates as age and previous ownership and adding and removing plates from the collection. To match this, further research in adaptive UX, HCI and IxD design can be needed.

The discussion above points towards the need for research on new design patterns to handle the temporality and the teach-learn qualities introduced by HIC-ML. The need here for HIC-ML based products designed and built with a contextual focus suggests the need for diversity in the design team that can reflect and encompass the domain targeted.

\subsection{Virtue ethics}

In the work, one base presumption is a situation where a domain expert not only has oversight but also makes decisions on context and subjectively defines a problem formulation. This approach, that couples human virtues to the use of ML is, as discussed in the introduction section, influenced by the technomoral virtues proposed by Vallor (2016). This is of course not a silver-bullet in the quest for trustworthy AI and does not provide any guarantee against misuse, but, it is an approach towards democratisation concerning the usage and towards a more widespread understanding of the 
capabilities of ML.

The focus on command as a governance principle has crystallised during the work and is a result of the type of design experiments selected. It is difficult, given this work, to envision how general this approach is for large scale systems. The battery design experiment, as well as, for example, the work by Lindvall et al. (2018), opens up for discussions concerning permission systems for a HIC-ML system that aim to share and create knowledge organically. These permission systems needs to, at a minimum, regulate and separate to what extent users and domain experts can add new knowledge to the HIC-ML system.

A consequence of the approach taken in this work is that any bias or misconception the domain expert has concerning the domain may be transferred to the model. The model will then be trustworthy only in a specific context or culture and it is up to the practical wisdom of the domain expert to use the HIC-ML system wisely.

\subsection{Limitations of the HIC-ML approach}

In situations where a goal can be defined from a objective perspective or in situations were labelled data is abundant, a data-driven approach is probably more effective than a HIC-ML approach. Recommender systems, language translation and games are areas where an objective function can be reasonably well-defined and consequently an HIC-ML approach less likely to be successfully. A HIC-ML approach might also, in these situations, be inefficient since the model building process is not optimised, as it can be for more well-defined problems where a large amount of labelled training data exists. In those cases, human-in-the-loop approaches on their own, like AL, IML and MT, can be effective to build accurate models and still open up for human involvement (See Section 3.8).

In this work, the aim was to use ML methods in the form of neural networks that creates internal representations of the domain knowledge in an inductive fashion based on the available training data (Se section 3.4). Knowledge priors in the battery sorter and the plate design experiment comes in the form of an imported pretrained model. These priors introduce bias to the HIC-ML model that can be difficult to lay bare and the decisions and explications can therefor be seductive.

The HIC-ML approach builds on that a domain expert sees enough value or meaning in the usage so it can be worth the effort to train the model and understand explications. In public-facing systems, for example, explaining 
why a loan did not go through or why an applicant did not get a position, this incentive does not exist and consequently, a HIC-ML approach adds little value.

\subsection{Future work}

The HIC-ML approach defined and discussed in this work can open up for the contextual shift in ML that Fei-Fei Li envisions in the opening quote. For this to happen and to open up for new products there will be a need for software development to build these systems that can enhance and augment human capabilities. This work point towards three central areas where more research and development is needed:

- Explicability: Build agents that can put forward evidence for a decision in a complex domain, as concepts, simplified surrogate models or lift out features important for a specific decision. Except for the more obvious ML challenges, challenges connected to IxD and HCI stands out.

- Teach-learn: In the commuter design experiment a simple domaindependent user interface for teaching was constructed. To target more complex domains user interfaces and design patterns as well as resource restrained training methods needs to be developed.

- Temporality: For a HIC-ML system there will be intensive teaching periods and periods where domain experts and users trust the system. In this work, explicability is lifted as a central factor. Among contextually related factors, dataset shift (Moreno-Torres et al., 2012) make once trustworthy decisions unreliable without any simple way for the domain expert to notice this. This type of challenges has to be addressed so contextually related changes can be identified and handled to underpin trustworthiness over time. 


\section{CONCLUSIONS}

The focus in this work is Machine Learning (ML) as an augmenting and enhancing technology for humans; an approach centred around human intelligence and a human in command that is liable. The goal is a setting that implies a human that uses unique human abilities to judge the ML system's limitations, understand and identify causal relations and do reasoning. Human in Command Machine Learning (HIC-ML) combine those human abilities with an ML system that can handle a large amount of data and promote decisions within a delimited domain. The approach implies that the HIC-ML system is designed so it can target a specific domain and consequently make use of human expertise related to that domain.

The results presented has emanated from a experiment-driven Research Through Design process with elements of Participatory Design. This initial explorative research approach gradually transforms and is replaced by the more utility-focused Design Science Research Methodology, used to formalise the results.

Results are presented as design guidelines for HIC-ML and an abstract model over the teach-learn process. Possible application areas are discussed for the design experiments and the notion of command as a governance principle. Finally, an approach to explain an ML system's decisions is presented, the approach uses the structure and rigidity of scientific explanations to clarify how human and machine capabilities and strengths can be combined to reach this goal. The approach to ML selected for this work grows from an awareness of the risks with AI. This work does not directly address ethically challenging domains. Instead, the HIC-ML framework builds on a human domain expert in command and promotes ethical considerations that rely on human virtues.

Future challenges include designing for interactivity and interaction in a dialogue sequence to reach an, for the human domain expert, acceptable explanation. The need for the HIC-ML system to communicate the explications' limitations and the domain wherein they can be trusted is also an important challenge. Central for the next step is to design an artefact where the target domain can be defined by the domain expert in which the proposed guidelines further can be assessed and developed. 



\section{REFERENCES}

Abdul, A., Vermeulen, J., Wang, D., Lim, B. Y., \& Kankanhalli, M. (2018). Trends and trajectories for explainable, accountable and intelligible systems: An HCI research agenda. In Conference on Human Factors in Computing Systems - Proceedings (Vol. 2018-April, pp. 1-18). New York, NY, USA: ACM. doi: 10.1145/3173574.3174156

Adadi, A., \& Berrada, M. (2018). Peeking Inside the Black-Box: A Survey on Explainable Artificial Intelligence (XAI). IEEE Access, 6, 52138-52160. doi: 10.1109/ACCESS.2018.2870052

Amershi, S., Begel, A., Bird, C., DeLine, R., Gall, H., Kamar, E., .. Z Zimmermann, T. (2019). Software Engineering for Machine Learning: A Case Study. In IEEE/ACM 41st International Conference on Software Engineering: Software Engineering in Practice (pp. 291-300). doi: 10.1109/ICSE-SEIP.2019.00042

Amershi, S., Cakmak, M., Knox, W. B., \& Kulesza, T. (2014). Power to the people: The role of humans in interactive machine learning. AI Magazine, 35(4), 105-120. doi: 10.1609/aimag.v35i4.2513

Amershi, S., Fogarty, J., Kapoor, A., \& Tan, D. (2011). Effective EndUser Interaction with Machine Learning. In Proceedings of the AAAI Conference on Artificial Intelligence (Vol. 25, No. 1).

Attenberg, J., Ipeirotis, P., \& Provost, F. (2015). Beat the machine: Challenging humans to find a predictive model's "unknown unknowns". Journal of Data and Information Quality, 6(1), 1-17. doi: 10.1145/2700832

Banovic, N., \& Krumm, J. (2017). Warming Up to Cold Start Personalization. PACM Interact. Mob. Wearable Ubiquitous Technol, 1(13). doi:10.1145/ 3161175

Barredo Arrieta, A., Díaz-Rodríguez, N., Del Ser, J., Bennetot, A., Tabik, S., Barbado, A., ... Herrera, F. (2020). Explainable Artificial Intelligence (XAI): Concepts, taxonomies, opportunities and challenges toward responsible AI. Information Fusion, 58, 82-115. doi: 10.1016/j.inffus .2019 .12 .012

Belle, V., \& Papantonis, I. (2020). Principles and practice of explainable machine learning. In arXiv preprint arXiv:2009.11698.

Bengio, Y., Jérôme Louradour, U., Collobert, R., \& Weston, J. (2009). Curriculum Learning. In Proceedings of the 26th annual International Conference on Machine Learning (pp. 41-48). ACM.

Biran, O., \& Cotton, C. (2017). Explanation and Justification in Machine Learning: A Survey. In IJCAI Workshop on Explainable AI (XAI) (Vol. 8, pp. 8-14). 
Boukhelifa, N., Bezerianos, A., \& Lutton, E. (2018). Evaluation of interactive machine learning systems. In Human and Machine Learning (pp. 341-360). Springer. doi: 10.1007/978-3-319-90403-017

Brundage, M., Avin, S., Wang, J., \& Belfield, H. (2020). Toward Trustworthy AI Development: Mechanisms for Supporting Verifiable Claims. arXiv preprint arXiv:2004.07213.

Cakmak, M., Chao, C., \& Thomaz, A. L. (2010). Designing Interactions for Robot Active Learners. Computing in Science \& Engineering, 2(2), $108-118$.

Camburu, O.-M. (2020). Explaining Deep Neural Networks (Doctoral dissertation, University of Oxford).

Carvalho, D. V., Pereira, E. M., \& Cardoso, J. S. (2019). Machine learning interpretability: A survey on methods and metrics. Electronics, 8(8), 832. doi: $10.3390 /$ electronics 8080832

Chernova, S., \& Thomaz, A. L. (2014). Robot learning from human teachers. Synthesis Lectures on Artificial Intelligence and Machine Learning, 8(3), $1-121$.

Chollet, F. (2019). On the Measure of Intelligence. arXiv preprint arXiv:1911.01547, 64.

Cohen, P., Cheyer, A., Horvitz, E., El Kaliouby, R., \& Whittaker, S. (2016). On the future of personal assistants. In Proceedings of the $2016 \mathrm{CHI}$ conference extended abstracts on human factors in computing systems (pp. 1032-1037). doi: 10.1145/2851581.2886425

Cooper, A. (1999). The Inmates Are Running the Asylum: Why High Tech Products Drive Us Crazy and How to Restore the Sanity. Indianapolis: Sams Indianapolis.

Dahlbäck, N., Jönsson, A., \& Ahrenberg, L. (1993). Wizard of Oz studies why and how. Knowledge-based systems, 6(4), 258-266.

Doshi-Velez, F., \& Kim, B. (2017). Towards a Rigorous Science of Interpretable Machine Learning. arXiv preprint arXiv: 1702.08608.

Dove, G., Halskov, K., Forlizzi, J., \& Zimmerman, J. (2017). UX Design Innovation: Challenges for Working with Machine Learning as a Design Material. In Proceedings of the 2017 CHI Conference on Human Factors in Computing Systems (pp. 278-288). doi: 10.1145/3025453.3025739

Dudley, J. J., \& Kristensson, P. O. (2018). A review of user interface design for interactive machine learning. ACM Transactions on Interactive Intelligent Systems, 8(2). doi: 10.1145/3185517

Fails, J. A., \& Olsen, D. R. (2003). Interactive Machine Learning. In Proceedings of the 8th International Conference on Intelligent User Interfaces (pp. 39-45). ACM. doi: 10.1145/604045.604056

Fazelpour, S., \& Lipton, Z. C. (2020). Algorithmic fairness from a non-ideal perspective. In AIES 2020 - Proceedings of the AAAI/ACM Conference on AI, Ethics, and Society (pp. 57-63). doi: 10.1145/3375627.3375828

Ferreira, A. C., Silva, L. R., Renna, F., Brandl, H. B., Renoult, J. P., Farine, D. R., ... Doutrelant, C. (2020). Deep learning-based methods for 
individual recognition in small birds. Methods in Ecology and Evolution, 11(9), 1072-1085. doi: 10.1111/2041-210X.13436

Gaver, W. (2012). What Should We Expect From Research Through Design? In Proceedings of the SIGCHI conference on human factors in computing systems (pp. 934-946).

Gilpin, L. H., Bau, D., Yuan, B. Z., Bajwa, A., Specter, M., \& Kagal, L. (2019). Explaining explanations: An overview of interpretability of machine learning. In Proceedings - 2018 IEEE 5th International Conference on Data Science and Advanced Analytics, DSAA 2018 (pp. 80-89). IEEE. doi: 10.1109/DSAA.2018.00018

Graneheim, U., \& Lundman, B. (2004). Qualitative content analysis in nursing research: concepts, procedures and measures to achieve trustworthiness. Nurse Education Today, 24(2), 105-112. doi: 10.1016/J.NEDT.2003.10 .001

Gudjonsdottir, R., \& Lindquist, S. (2008). Personas and scenarios: Design tool or a communication device? From CSCW to Web 2.0: European Developments in Collaborative Design Selected Papers from COOP08.

Guidotti, R., Monreale, A., Ruggieri, S., Turini, F., Giannotti, F., \& Pedreschi, D. (2018). A survey of methods for explaining black box models. ACM Computing Surveys, 51(5), 42. doi: 10.1145/3236009

Gunning, D. (2017). Explainable artificial intelligence (xai). Defense Advanced Research Projects Agency (DARPA), nd Web, 2, 2.

Herriott, R. (2019). What kind of research is research through design? In International Association of Societies of Design Research Conference 2019 (p. 11).

Hevner, A. R., March, S. T., Park, J., \& Ram, S. (2004). Design Science in Information Systems Research. MIS Quarterly, 28(1), 75-105. doi: 10 $.2307 / 25148625$

Hilton, D. J. (1990). Conversational processes and causal explanation. Psychological Bulletin, 107(1), 65-81. doi: 10.1037/0033-2909.107.1 .65

HLEG. (2019). Ethics Guidelines for Trustworthy AI. European Commission, 32(May), 1-39.

Jobin, A., Ienca, M., \& Vayena, E. (2019). The global landscape of AI ethics guidelines. Nature Machine Intelligence. doi: 10.1038/s42256-019-0088 $-2$

Konyushkova, K., Sznitman, R., \& Fua, P. (2017). Learning Active Learning from Data. Learning from Data: Concepts, Theory, and Methods: Second Edition, 1-538. doi: 10.1002/9780470140529

Krippendorf, K. (2005). The semantic turn: A new foundation for design. Boca Raton: crc Press.

Kulesza, T., Burnett, M., Wong, W. K., \& Stumpf, S. (2015). Principles of Explanatory Debugging to personalize interactive machine learning. In Proceedings of the 20th International Conference on Intelligent User Interfaces (Vol. 2015-Janua, pp. 126-137). Association for Computing 
Machinery. doi: 10.1145/2678025.2701399

Lakkaraju, H., Bach, S. H., \& Leskovec, J. (2016). Interpretable decision sets: A joint framework for description and prediction. In ACM SIGKDD International Conference on Knowledge Discovery and Data Mining (pp. 1675-1684). doi: 10.1145/2939672.2939874

Lakkaraju, H., \& Bastani, O. (2020). "How do i fool you?": Manipulating user trust via misleading black box explanations. In AIES 2020 - Proceedings of the AAAI/ACM Conference on AI, Ethics, and Society (pp. 79-85). doi: $10.1145 / 3375627.3375833$

Lakkaraju, H., Kamar, E., Caruana, R., \& Horvitz, E. (2017). Identifying unknown unknowns in the open world: Representations and policies for guided exploration. In Proceedings of the AAAI Conference on Artificial Intelligence (Vol. 31, No. 1) (pp. 2124-2132). AAAI.

Lakkaraju, H., Kamar, E., Caruana, R., \& Leskovec, J. (2019). Faithful and Customizable Explanations of Black Box Models. In Proceedings of the 2019 AAAI/ACM Conference on AI, Ethics, and Society (pp. 131-138) (pp. 131-138). Honolulu: ACM.

Lawton, M. P., \& Brody, E. M. (1969). Assessment of Older People: Self-Maintaining and Instrumental Activities of Daily Living. The gerontologist, 9(3 Part 1), 179-186.

Lecun, Y., Bengio, Y., \& Hinton, G. (2015). Deep learning. Nature, 521(7553), 436-444. doi: 10.1038/nature 14539

Leslie, D. (2019). Understanding artificial intelligence ethics and safety: A guide for the responsible design and implementation of AI systems in the public sector. The Alan Turing Institute.

Lindvall, M., Molin, J., \& Löwgren, J. (2018). From machine learning to machine teaching. Interactions, 25(6), 52-57. doi: 10.1145/3282860

Lipton, Z. C. (2016). The Mythos of Model Interpretability. Communications of the ACM, 61(10), 35-43. doi: 10.1145/3233231

Lundberg, S. M., \& Lee, S. I. (2017). A unified approach to interpreting model predictions. In Advances in Neural Information Processing Systems (pp. 4766-4775). MIT press.

Meek, C., Simard, P., \& Zhu, X. (2016). Analysis of a Design Pattern for Teaching with Features and Labels. arXiv preprint arXiv 1611.05950.

Miller, T. (2019). Explanation in artificial intelligence: Insights from the social sciences. Artificial Intelligence, 267, 1-38. doi: 10.1016/j.artint.2018 .07 .007

Moreno-Torres, J. G., Raeder, T., Alaiz-Rodríguez, R., Chawla, N. V., \& Herrera, F. (2012). A unifying view on dataset shift in classification. Pattern Recognition, 45(1), 521-530. doi: 10.1016/j.patcog.2011.06.019

Mueller, S. T., Hoffman, R. R., Clancey, W., Emrey, A., \& Klein, G. (2019). Explanation in Human-AI Systems: A Literature Meta-Review, Synopsis of Key Ideas and Publications, and Bibliography for Explainable AI. arXiv preprint arXiv:1902.01876. 
Munro, R. (2021). Human-in-the-Loop Machine Learning. Manning Publications.

Newcomb, T., \& Heider, F. (1958). The Psychology of Interpersonal Relations. American Sociological Review, 23(6), 742. doi: 10.2307/2089062

Nielsen, L. (2013). Personas - User Focused Design. Springer. doi: 10.1007/ 978-1-4471-4084-9

Norman, D. A. (1983). Some observations on mental models. Mental models, 7(112), 7-14.

Otto, M. (2018). Regulation (EU) 2016/679 on the protection of natural persons with regard to the processing of personal data and on the free movement of such data (General Data Protection Regulation - GDPR). In International and European Labour Law (pp. 958-981).

Overton, J. A. (2012). Explanation in Science (Doctoral dissertation, University of Western Ontario).

Peffers, K., Tuunanen, T., Rothenberger, M. A., \& Chatterjee, S. (2007). A Design Science Research Methodology for Information Systems Research. Journal of Management Information Systems, 24(3), 45-77. doi: 10.2753/MIS0742-1222240302

Rahwan, I., Cebrian, M., Obradovich, N., Bongard, J., Bonnefon, J.-F., Breazeal, C., ... Wellman, M. (2019). Machine behaviour. Nature, 568(7753), 477-486. doi: 10.1038/s41586-019-1138-y

Ramos, G., Meek, C., Simard, P., Suh, J., \& Ghorashi, S. (2020). Interactive machine teaching: a human-centered approach to building machine-learned models. Human-Computer Interaction, 35(5-6), 413451. doi: 10.1080/07370024.2020.1734931

Ravichandar, H., Polydoros, A. S., Chernova, S., \& Billard, A. (2020). Recent Advances in Robot Learning from Demonstration. Annual Review of Control, Robotics, and Autonomous Systems, 3(1), 297-330. doi: 10.1146/annurev-control-100819-063206

Ribeiro, M. T., Singh, S., \& Guestrin, C. (2016). "Why should i trust you?" Explaining the predictions of any classifier. In Proceedings of the 22nd ACM SIGKDD International Conference on Knowledge Discovery and Data Mining (Vol. 13-17, pp. 1135-1144). ACM. doi: 10.1145/ 2939672.2939778

Ribera, M., \& Lapedriza, A. (2019). Can we do better explanations? A proposal of user-centered explainable AI. In IUI Workshops (Vol. 2327, p. 38).

Rittel, H. W., \& Webber, M. M. (1973). Dilemmas in a general theory of planning. Policy Sciences, 4(2), 155-169. doi: 10.1007/BF01405730

Robertson, Toni, Simonsen, \& Jesper. (2012). Routledge International Handbook of Participatory Design. Routledge.

Roscher, R., Bohn, B., Duarte, M. F., \& Garcke, J. (2020). Explainable Machine Learning for Scientific Insights and Discoveries. IEEE Access, 8, 42200-42216. doi: 10.1109/ACCESS.2020.2976199

Selvaraju, R. R., Cogswell, M., Das, A., Vedantam, R., Parikh, D., \& Batra, D. (2020). Grad-CAM: Visual Explanations from Deep Networks via 
Gradient-Based Localization. International Journal of Computer Vision, 128(2), 336-359. doi: 10.1007/s11263-019-01228-7

Settles, B. (2010). Active Learning Literature Survey (Tech. Rep. No. January). University of Wisconsin-Madison.

Simard, P. Y., Amershi, S., Chickering, D. M., Pelton, A. E., Ghorashi, S., Meek, C., ... Wernsing, J. (2017). Machine Teaching: A New Paradigm for Building Machine Learning Systems. arXiv preprint arXiv:1707.06742.

Stappers, P., \& Giaccardi, E. (2017). Research through Design. In The Encyclopedia of Human-Computer Interaction (pp. 1-94). The Interaction Design Foundation.

Stappers, P. J. (2007). Doing design as a part of doing research. In Design research now (pp. 81-91). Springer.

Star, S. L. (2010). This is not a boundary object: Reflections on the origin of a concept. Science Technology and Human Values, 35(5), 601-617. doi: 10.1177/0162243910377624

Tjoa, E., \& Guan, C. (2019). A Survey on Explainable Artificial Intelligence (XAI): Towards Medical XAI. In IEEE Transactions on Neural Networks and Learning Systems.

Vallor, S. (2016). Technology and the virtues: A philosophical guide to a future worth wanting. Oxford University Press.

Winograd, T., Flores, F., \& Flores, F. F. (1986). Understanding computers and cognition: A new foundation for design. Intellect Books.

Woodward, J. (2019). Scientific Explanation. In The Stanford Encyclopedia of Philosophy.

Yang, Q. (2018). Machine Learning as a UX Design Material: How Can We Imagine Beyond Automation, Recommenders, and Reminders? 2018 AAAI Spring Symposium Series, 467-472.

Zhu, X. (2015). Machine Teaching: An Inverse Problem to Machine Learning and an Approach Toward Optimal Education. In Proceedings of the Twenty-Ninth AAAI Conference on Artificial Intelligenc (pp. 4083-4087).

Zhu, X., Singla, A., Zilles, S., \& Rafferty, A. N. (2018). An Overview of Machine Teaching. arXiv preprint arXiv:1801.05927.

Zimmerman, J., Stolterman, E., \& Forlizzi, J. (2010). An analysis and critique of Research through Design. Proceedings of the 8th ACM Conference on Designing Interactive Systems, 310. doi: 10.1145/1858171.1858228 



\section{Glossary}

artificial intelligence Intelligence demonstrated by machines.

black box An ML model where no intelligible relation between the input features and the promoted decision exists.

boundary object Design experiments that are temporal, based in action, subject to reflection and local tailoring (Star, 2010).

causal attribution A static relation that ascribes a decision to its origin for an ML system. Causal attributions can consequently function as evidence for a decision (Miller, 2019; Hilton, 1990). If a causal attribution is lifted out in comparison with other causal attributions they are denoted contrastive attributions.

causal explanation A causal explanation in human only contexts includes interpersonal exchange and are given: implying that someone explains something to someone. An explanation is often initiated by a why question formulated by an explainee (Hilton, 1990).

confusion matrix A table layout that allows visualisation of the performance of an ML method.

contrastive attribution Contrastive attributions are causal but builds on a comparison between causal attributions that in some sense are similar.

contrastive explanation A causal explanation that answer to a why-not question. A general format is: why did A happen instead of B. In a causal explanation B is often implicit (Hilton, 1990).

decision The output from an ML system in the form of a classification, recommendation, prediction, proposed decision or action.

design team A team of professionals that includes competences needed to build an HIC-ML system. Includes for example: domain expert, 
programmers, ML expert, UX and IxD designers, graphic designers and project managers.

domain expert A person that knows, at least partly, the domain the ML system targets, and can, at least partly, understand and evaluate a proposed decision. These persons are also users. They also believe the HIC-ML system can add some type of value or meaning and therefor are willing to invest time and effort in order to train the system and understand its decisions.

example Data used to train a model. An example represents an aspect of the real world. Examples can be raw in the form of images, text or sound or consist of features. The raw format implies that the interdependence between the discrete parts of the input data is high. Consequently one discrete part carry information only in its context. For example one letter in a text or one pixel in an image.

explainability ML systems that are able to summarise the reasons for their behaviour, gain the trust of users, or produce insights about the causes of their decision.

explainee A human that tries to understand the explication an ML explicator promotes.

explanandum All possible causal explanations that can be promoted by the HIC-ML together with the domain expert system as evidence for a decision.

explicability ML systems that are able to lift out evidence for a decision understandable for a domain expert.

explicandum All possible causal attributions that can be promoted by the HIC-ML system as evidence for a decision.

explication Causal attributions presented for the explainee related to a decision. The explication is static and expressed using the domains ontology and does not imply any interpersonal exchange as an explanation does.

feature One example consist of one to many features. For example location, time and date. Features are often engineered and independent of other features. 
feature space The mathematical space that consists of all possible values of the features.

HIC-ML Human In Command - Machine Learning: implies that a domain expert decides the target domain and to some extent can utilise the teach-learn capabilities of ML. Domain experts are then responsible for direction, purpose and ethical considerations concerning the usage of an ML system.

HITL-ML Human In The Loop - Machine Learning: Human interaction with machine learning systems to improve human performance, machine performance, or both.

interpretability The degree humans can understand the cause of a decision made by an ML system.

MITL-ML Machine In The Loop - Machine Learning: Human authority over machine learning systems in order to add meaning and value to humans.

ML Machine learning, computer algorithms that improve automatically when they are exposed to training data. ML is subset of AI.

ML expert A person that can select ML algorithm, build and train an ML model so it fulfils a given a problem formulation.

ML explicator A ML system that can promote an explication to a human explainee.

ML model Machine learning involves selecting a method/algorithm useful to train a ML model. The ML method selected has to match a problem definition. The model is then trained with data representative for the the deployment domain.

ML system The ML system consists of the ML model and computational resources needed to handle input data and present promoted decisions.

model space Model space or hypothesis space is the set of all possible models that can be trained given a feature space and a ML model. 
neural network A collective term that includes all type of ML methods that build on artificial neurons (for example ANN, DNN, CNN, GAN and RNN).

open-ended problem Those problems that have many solutions or no solutions for the problem as defined.

personal knowledge domain Knowledge in a personal domain is not useful if transferred from one user to another, for example a users address, relatives or commute patterns.

ResNet Deep residual neural networks trained on the ImageNet dataset. ImageNet is a public dataset that consists of around 14 million handlabelled images. The number in ResNet34 and ResNet50 indicates the number of layers in the neural network. These pretrained networks are useful in a transfer learning setting in that they allow the transfer of low-level internal image representations (colours, basic forms and shapes) from one target domain to another. This, in turn, reduces the need for training data in the target domain.

sand-boxed A highly controlled environment.

scientific explanation In a simple form it is an answer to a why-question, a form of explanation that explain something we see in the natural world based on observations and measurements (Woodward, 2019).

shared knowledge domain A domain were at least part of knowledge is shared between users. For example, plants in Sweden.

training data Data used to train a model. The data represents an aspect of the real world and consists of examples.

transfer learning When a trained ML model is applied and retrained to target a problem similar to the problem it was initially trained on.

user A person that believes a HIC-ML system can add some type of value or meaning to them. 


\section{Acronyms}

AI Artificial Intelligence.

DSRM Design Science Research Methodology.

IxD Interaction Design.

ML Machine Learning.

PD Participatory Design.

RtD Research through Design.

UX User Experience. 



\section{PART II.}

PAPERS 



\section{PAPER 1 - EVALUATING \\ INTERPRETABILITY IN \\ MACHINE TEACHING}

\section{Lars Holmberg, Paul Davidsson and Per Linde}

\section{Abstract}

Building interpretable machine learning agents is a challenge that needs to be addressed to make the agents trustworthy and align the usage of the technology with human values. In this work, we focus on how to evaluate interpretability in a machine teaching setting, a setting that involves a human domain expert as a teacher in relation to a machine learning agent. By using a prototype in a study, we discuss the interpretability definition and show how interpretability can be evaluated on a functional-, humanand application level. We end the paper by discussing open questions and suggestions on how our results can be transferable to other domains. 



\section{Evaluating Interpretability in Machine Teaching}

\subsection{Introduction}

A missing keystone in the quest of building trustworthy AI agents is the ability for the agent to present results that are explainable and interpretable. Interpretability put demands on a human to be able to understand the result, explainability put demands on that the result is presented so it can be understood and traced by a human being (HLEG, 2019).

Whilst interpretability of machine learning, from a computer science perspective, is an emerging field (Doshi-Velez \& Kim, 2017; Gilpin et al., 2019; Lakkaraju et al., 2016). There is a growing interest in addressing interpretability through a model-agnostic approach (Ribeiro et al., 2016; Teso \& Kersting, 2019; Lundberg \& Lee, 2016; Lakkaraju et al., 2019; Hind et al., 2018) but there are to our knowledge a lack of real world case studies.

In this work, we explore how interpretability can be evaluated in a machine learning process that uses the paradigm of Machine Teaching (MT) (Simard et al., 2017). A setting where a human teacher is in control of the machine learning process.

We will consequently focus on the following research question.

- How can interpretability be evaluated for an MT agent?

To shed light on this question, we created an MT prototype targeting the personal knowledge domain commuting. As a domain, commuting is well-known even if commute patterns are individual and a commuter is the only expert in their patterns. A commuter, using our prototype, can teach the agent selected commutes. Results in this area can give indication and inspiration regarding future studies in shared domains, for example, radiology (Lindvall et al., 2018) or personal domains, for example, personalisation of intelligent personal assistants and assistive technology.

We aimed at evaluating interpretability inspired by the considerations that Doshi-Velez and Kim (2018) outline. Our results are in line with that work, but our work adds practical knowledge on how the considerations 
can be applied and used in a real setting. We also unpack local and global interpretability in relation to evaluation. Our focus also add awareness around how explanations and interpretability relates to the end user of the agent. We do this by drawing attention to the term explicability, in this work defined as a term between interpretability and explanations that implies that a domain expert, on their own, can formulate an explanation for an explicable agent.

We proceed as follows, first, we give an overview of related work and introduce our approach. We then outline the study setup that we evaluate in a result and analysis section. Before concluding the results are elaborated in a discussion section.

\subsection{Related work}

The report Ethics guidelines for Trustworthy AI (HLEG, 2019) highlights three components of AI agents: ethical, robust and lawful. Important for the ethical component is that the agent is explicable, implying a transparency that makes the agent explainable and contestable to those affected (HLEG, 2019). In the report, the terms explicability and explicable are used, according to Merriam-Webster explicable means capable of being explained ${ }^{1}$. The report also highlights that demands concerning explicability is highly dependent on the context and severity of the consequences. In this work, we will have a focus on interpretability, explicability, and explainability as an important part of the ethical component and less focus on the components robustness and lawful.

One view on interpretability is the coupling to transparent algorithms, like linear models or decision trees, algorithms that can make the entire model transparent and simultaneity is possible for a human. This coupling is contested (Lipton, 2016; Lou et al., 2012) in that interpretability challenges has less to do with the choice of model and are more connected to the complexity of the agent and thus, that, for example, linear models are not automatically interpretable and neural networks not automatically black-boxes from an explainability perspective. If the features and labels used in a neural network are understandable, a human can in some cases explain the predictions using the relation between feature values and labels, even if the network as such is a black box.

In an overview of explanations and justification in machine learning Biran and Cotton (2017) provides this definition of explainability and inter-

${ }^{1}$ Merriam-Webster dictionary, accessed 2020-01-24 
pretability:

Explanation is closely related to the concept of interpretability: systems are interpretable if their operations can be understood by a human, either through introspection or through a produced explanation.

In this work, we define an end user as anyone that uses the system, a usage that does not demand any ML or domain expertise. Among these end users, there are domain experts/teachers with a teaching goal, this goal is manifested as a knowledge transfer from the teacher to the ML agent. This transfer is performed without any need for ML expertise. We use the terms domain expert and teacher interchangeable.

There exists, in related literature, a limited discussion on requirements on the end user's capability to understand the explanation, although the complexity in providing good explanations are acknowledged (DoshiVelez \& Kim, 2018; Miller, 2019). In this work, we will focus on the end user and therefore we distinguish between interpretability, explicability, and explainability in relation to the end user. We define interpretability as a property of the agent in line with the Biran and Cotton (2017) definition, which can imply a need for Machine Learning (ML) expertise and/or domain expertise to produce an, for an end user, understandable explanation. For an explicable ML agent, we define that, a domain expert is needed to adapt or rephrase the explanation in understandable terms to the end user. Explainability is then, as we define it, a quality of an ML agent that does not need any human intermediary to adapt or rephrase an explanation to be understood by an end user. An ML agent can then provide, depending on the situation, predictions that are interpretable, explicable or formulate an explanation concerning the prediction. Implementing agents that are capable of providing explanations implies understanding what constitutes a good explanation (Miller, 2019). The focus in this work is, instead of the problem of formulating good explanations, on interpretability and explicability and ML systems that involves a human domain expert capable of formulating explanations.

Doshi-Velez and Kim (2018) emphasize that a need for interpretability builds on downstream goals as fairness, privacy, reliability, robustness, causality, usability and trust. The authors also urge researchers to both define these goals as well as question if interpretability is the right tool to achieve these goals. Interpretability is seen as a quality needed when the problem formulation is incomplete. The authors also state three 
modes of interpretability evaluation with increasing specificity and cost: Functionally-grounded evaluation with no real humans and proxy tasks, Human-grounded evaluation with real humans and simple tasks and finally Application-grounded evaluation with real humans and real tasks.

\subsubsection{Machine teaching}

Simard et al. (2017) primarily sees MT as a paradigm shift decoupling the domain experts from the ML experts in order to build systems that can be trained and deployed and used without the involvement of ML experts. In that work, the importance of a domain-specific language is highlighted, a language that builds on the taxonomy for the specific domain. MT is then, as we define it, an approach that gives a domain expert control over subjectively selected knowledge to be transferred to a model in a machine learning agent using a domain-specific language. An important part of this language is the user interface that facilitates human-agent interaction.

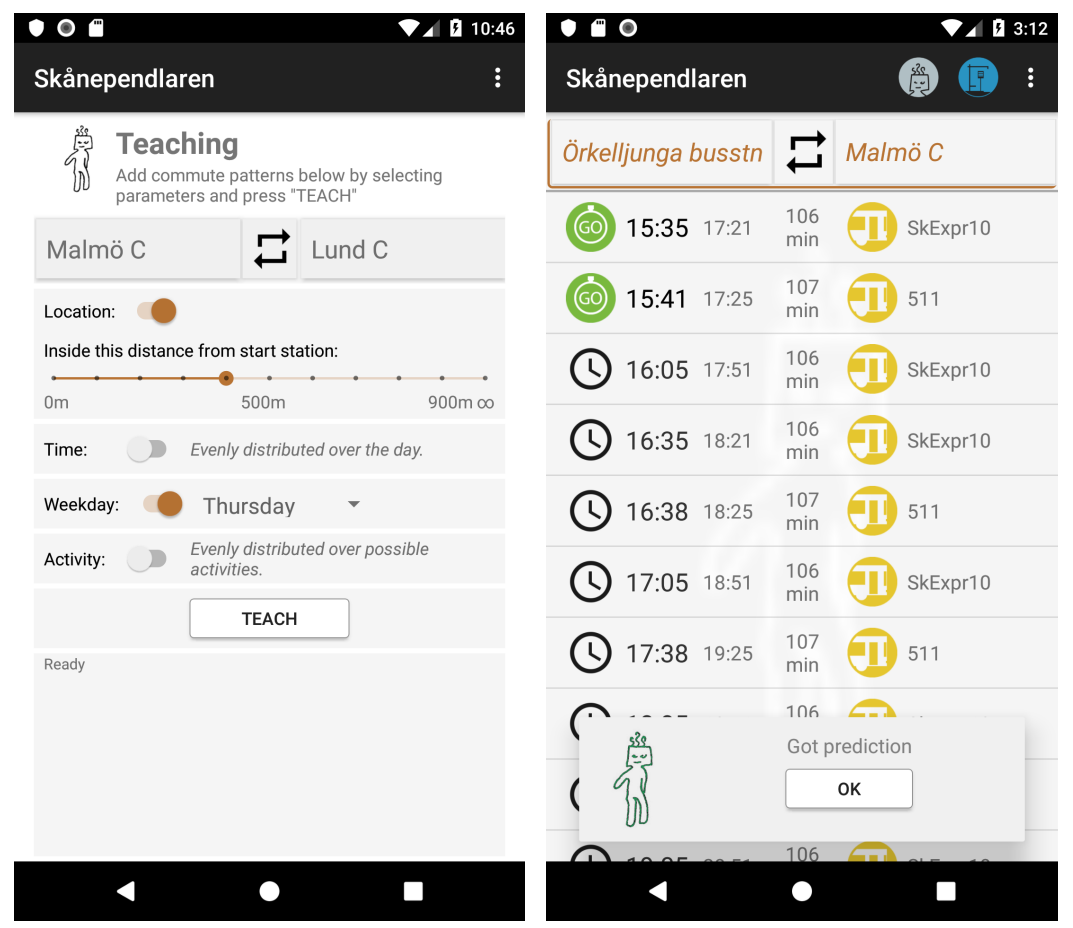

Figure 1: To the left, the apps teaching interface is shown and to the right, the interface for the predictions is shown. 


\subsection{Research setting}

The domain commuting has some qualities that makes it interesting as a research domain in the area of interpretable MT, the domain is wellknown, accessible but still commute patterns are individual. This implies that a commuter is domain expert in their commute patterns. We built a functioning prototype for the commuting domain that consist of a MT interface and a prediction interface. The final design can be seen in fig. 1 .

An important part of any machine learning implementation is the selection of features to use. This is also central in an MT implementation since an available orthogonal feature adds an extra dimension to the feature space and thus gives the teacher further possibilities to separate classes. For commuting the end user's location, day and time seemed to be a natural selection, we also added the end user's activity (still, walking running, in vehicle) as a feature. Since we are interested in commuting we chose the complete journey as our label, consisting of an origin-destination pair.

Neural networks can handle multiple levels of representation and raw input data (Lecun et al., 2015; Hornik et al., 1989). By selecting neural networks as our ML method we can simplify interpretation since the learner and the commuter uses the same, for a domain expert, understandable features and labels without any feature engineering. We decided to use a neural network framework from fast.ai ${ }^{2}$ since that framework is open source and aims at simplifying experimentation and rapid prototyping. We used the fast.ai tabular application and the automatic learning rate finder implemented in fast.ai.

The black box property of neural networks is a challenge since interpretability only can be evaluated on a agent level and not on a model level. The black box property forces us to aim for model agnostic interpretability which is in line with a growing research interest (Ribeiro et al., 2016; Teso \& Kersting, 2019; Lundberg \& Lee, 2016; Lakkaraju et al., 2019; Hind et al., 2018). Data-hunger is another property of neural networks that has to be mitigated. In a setting like ours, that is cold started, augmented and/or synthetic training data needs to be created during the teaching sessions. For the ML-pipeline in our implementation an online database (Firebase) is used for communicating with the end user's Android applications, a Node.js server orchestrates training, prediction and deployment by communicating with a Flask server and the online database. The fast.ai models, one for each commuter, are hosted on the Flask server.

\footnotetext{
${ }^{2}$ fast.ai
} 
Our initial general target scenario was: a commuter that starts the Android application and expects a journey prediction within a few seconds. Before evaluating interpretability we made sure that training time and prediction time were short enough to be useful in the target scenario. With our ML pipeline and a small neural network we reached prediction times in the area of a few seconds a retraining time around 15 seconds, we assessed these metrics as sufficient for the study.

\subsection{Methodology}

To evaluate interpretability from a functional and human perspective we created three personas (Nielsen, 2013) ${ }^{3}$ based on personas created for a local transport provider. For interpretability evaluation on an application level, we conducted an eight-week study. As study participants, we selected eight participants, four males, and four females, with experience from computer science and interaction design. Two were from the industry and six from the University. The participants used the app daily and we conducted six meetings and workshops. At the meetings, we discussed and compared experiences from last week and discussed the tasks for next week. We analyzed recorded interviews using content analysis (Graneheim \& Lundman, 2004) focusing on comments on interpretability. The participants were reimbursed by free journeys during the study.

\subsection{Result and Analysis}

In this section, we describe the process of evaluating interpretability for the prototype. The steps in this process are presented in a chronological order and for each step we move closer to application level evaluation. The focus is on producing predictions that full-fills the downstream goals of being causal, trustworthy and usable. Causal so that predictions map the commuters intentions, trustworthy and usable so the predictions reflect the context cue of the commuter. Our problem formulation is incomplete and can thus benefit from being interpretable since individual commute patterns cannot be defined in advance and are subject to change (DoshiVelez \& Kim, 2018).

For the prototype, we aimed for an explicable agent, for which, a domain expert/commuter can formulate explanations for performed predictions. For example, based on a given journey prediction, a commuter could formulate the following explanation "Since it is Tuesday evening I am

\footnotetext{
${ }^{3}$ https://github.com/k3larra/commuter\#personas
} 
going to town to meet Johan and play boule". When predictions do not match the commuter's expectations there could be three types of reasons. Either, since it is an MT setting, the commute pattern has not been taught to the agent, the commute patterns shifted or the agent is not robust and cannot provide an explicable prediction. The information that is intended to be explicable consists of the predicted journey and the context cue (location, time, day and activity).

\subsection{Functionally-grounded Evaluation: no humans, proxy tasks}

In this more general and global evaluation, we aimed to make predictions interpretable in relation to the different features. In this MT setting no data exists initially to train from, and the data is created in a teaching session by selecting sub-spaces in the feature space using the interface in figure 1 .

We experimented and found that using two hidden layers (200|100 neurons), and creating under 100 examples inside the sub-space made it possible for the training to converge in less than 20 epochs ${ }^{4}$. With this setting, retraining time is still under 30 seconds for training data consisting of up to a few thousand rows on a non GPU cloud server.

We then evaluated the interpretability by keeping all features constant except one so we could investigate how the generalization maps to interpretability. A visualization of one experiment can be seen in figure 2 where all features except location is kept constant and four different journeys are predicted, represented by the different colors. The images shows a situation where the generalization is designed so a journey should be predicted from the closest station.

In the scenarios we created for our personas we defined that a commuter only wants to make predictions centred around an origin station. An alternative edge scenario could be a commuter that wants to teach the prototype to make predictions for someone else, for example, a child, based on a location different from the origin station's location. The result would be, if for example more red teaching data (figure 2) was placed at a location to the left, that classification boundaries would be more complex. To avoid this, since we judged that this would result in predictions that would not be explicable, we only allowed one location, with user-defined size, surrounding the origin station for a journey.

We made similar tests and reasoning using scenarios concerning the

\footnotetext{
${ }^{4}$ https://github.com/k3larra/commuter/blob/master/machine_teaching/mt.ipynb
} 
other features, but made no restrictions. As an example, if a journey is taught on Monday mornings we deemed that a logical generalization would be to predict the same journey on all weekdays when no other conflicting journeys exists.

The functional test ended with daily usage by the development team to verify the robustness of the implementation.
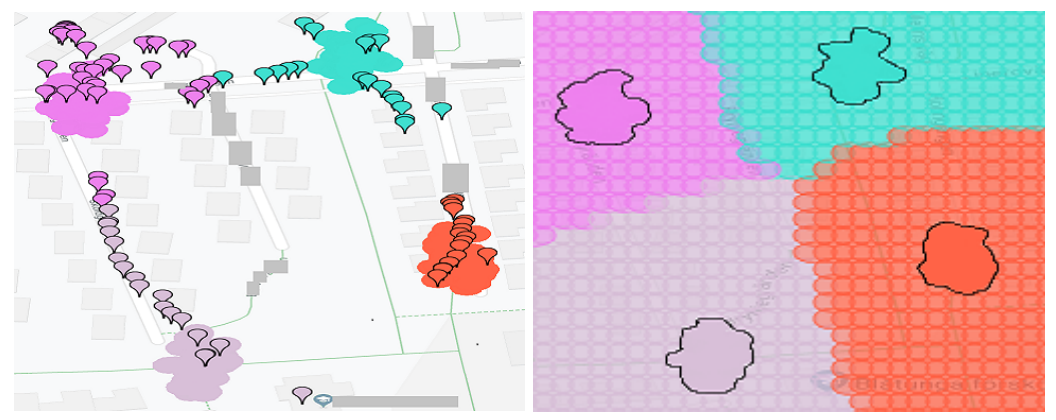

Figure 2: Location feature space, markers represent predictions, blobs represent teaching data and areas are classification boundaries.

\subsection{Human-grounded evaluation: proxy humans, sim- plified tasks}

Compared to the interpretability evaluation that Doshi-Velez and Kim (2018) names as human grounded using real humans and simplified task we saw a need for a more controllable and reproducible evaluation than using real humans could give. Using the design team for this was time-consuming and prone to errors, for example, inaccuracy in GPS position and delays in transport made it difficult to reproduce tests. Here our personas were used as a trade-off. For each of our personas, we initially created three scenarios and trained our agent using the teaching interface to make predictions matching the scenarios. A scenario formulated for the persona Andrea 5 can be read below:

It is Monday morning 7:23 and Andrea is as usual late for the bus and runs towards the bus stop "Veberöd försköningen". She checks the app while running to see if there are any delays.

To get more realistic data we generated three datasets that mimics oneyear journeys for the personas. These datasets were used as our test sets to

${ }^{5}$ https://github.com/k3larra/commuter\#personas 
evaluate the model's performance. We used our teaching interface to teach the agent and evaluated the result using visual representations as those that can be seen in figure 3. Metrics around how many epochs/time the training needs was balanced towards the amount of teaching data we created (the bar graph). A confusion matrix was used to visualize journeys that are not explicable. Based on these tests the final configurations for the ML pipeline and back-end were decided. (Two hidden layers with (200|100 neurons), 40 synthetic examples created evenly distributed inside the teaching subspace and training over 7 epochs $^{6}$.)

Decisions at this level has consequences for the interpretability inbetween global and local since we focus on results based on data for our personas. The diversity and complexity of the commute patterns for the personas has a normative influence for the behavior of the agent.
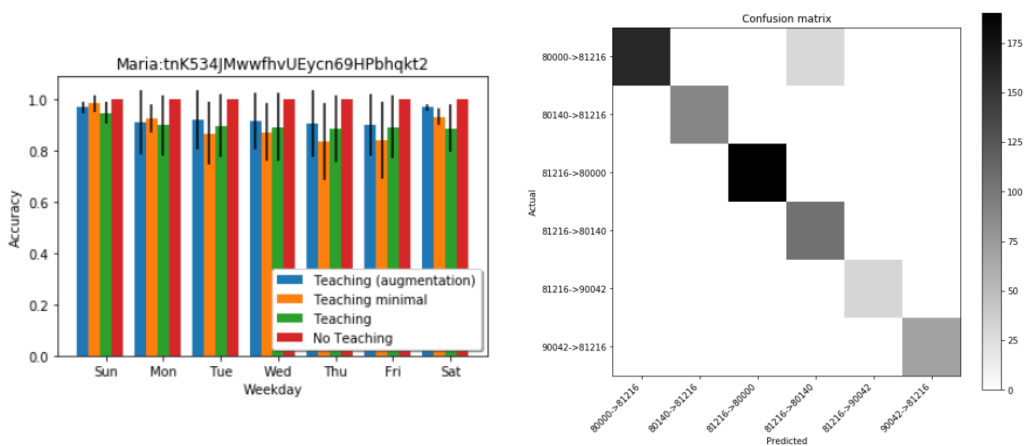

Figure 3: Tools used during human grounded interpretability evaluation. The test uses synthetic data generated for an envisioned one-year usage for the personas. The data was created using the teaching interface (figure 1). To the left accuracy and variance is aggregated over the week using different amount of generated teaching data (No teaching indicates a baseline), to the right precision is mapped in the form of a confusion matrix.

\subsection{Application-grounded evaluation: real humans, real tasks}

This part of the interpretability evaluation involves real humans and real tasks. A consequence of our MT setting is that it contains a temporal aspect in the form of teaching strategies. The participants explored different strategies, for example, teaching once out of context in the beginning of the week, teaching only when in context, if the predictions were wrong and a combination of these strategies. The focus for this phase was on

${ }^{6}$ https://github.com/k3larra/commuter/blob/master/machine_teaching/mt.ipynb 
local explicability, the situation when the end user compares the predicted journey with the intended journey and current context cue.

The participants, in general, found the predictions interpretable in relation to the commute patterns they already taught, or as expressed by one of the participants:

But it did correct .. around half of the times it was wrong. So it is still logic. Even if i doesn't favor me so to say.

The predictions generalized as intended in the sense that if a journey was taught on one day at a specific location and time it was predicted for all other days at that time. We discussed this inherent quality in machine learning compared to applications based on traditional algorithmic programming. We especially discussed the situation when predictions are made with low class probability and how this should be handled. One participant expressed that predictions should only be given "close" to the teaching data:

But I would like to say that if you are under some percentage .. and the app has really no idea of where you want to go.. that a dialog comes up.. or that a question-mark is shown to say "I do not know please teach me where you want to go right now"

Over time and with extensive teaching the study participants found that the predictions given deviated from the commute patterns taught and thus the predictions were not explicable. There are different explanations for this. Firstly, our prototype was built and tested using quite simple commute patterns based on scenarios and personas and those do not match more complex commute patterns. Secondly, the deviation can stem from the fact that, in the current implementation, teaching cannot be undone if mistakes in the teaching is made and erroneous journeys are taught.

\subsection{Discussion}

In this work we focus on post-hoc agent-level explicability a situation were a human domain expert can explain the predictions given contextual features like time, day and location and previous teaching. In our setting, we have a one to one relationship between a human domain expert and a machine learning agent. The agent is initially untrained and designed to target the commuting domain.

With our work we add to the interpretability terminology by Lipton (2016) and Doshi-Velez and Kim (2018). We use the term explicability to 
indicate a quality of the agent and distinguish between predictions that are interpretable and those that are explicable. Interpretable predictions does, with this distinction, need both ML expertise and domain expertise to be explained whilst explicable predictions can be explained using domain expertise.

When our prototype is used the end user can, for an explicable prediction, formulate an explanation that matches the intended journey. If the prototype is robust, and the wrong journey is predicted, this can be because it has not yet been taught the commute pattern or that the user for one or another reason chose not to teach the pattern. For our prototype, this local in context explicability, works well for simple commute patterns. A possibility to assess the model's knowledge from a global perspective and not only locally and in context would be an important addition from an explicability perspective.

We designed our prototype with the intention of maximizing the part of the predictions that are explicable. By evaluating interpretability during the design phase from a functional-, human- and application level, we found, in line with Doshi-Velez and Kim (2018) that the cost and specificity increases with those levels. From a functional level, it was clear that design consideration had a global impact on the final design. By using personas (Nielsen, 2013) instead of real humans in the human level evaluation we could strike a balance between a human perspective and still get reproducible results. The impact from this evaluation, which sits between a global and local perspective, to a large extent defines the portions of predictions that in the final application are explicable. A larger and more diverse collection of personas with more complex commute patterns would in our case produce a prototype that is explicable for a more diverse user group. The application-level evaluation gave us insights into the temporal aspect of the model's taught knowledge and explicability on a local level.

Over time and in more complex teaching environments it will be crucial to assess the learner, in our case a short description, perhaps in the form of a global explanation, using interpretable decision set (Lakkaraju et al., 2016) could be enough like "If weekday=Yes and Location=A and Time $>18: 00$ and Time $<22: 00$ then travel from A to B" to make the predictions explicable. If the areas in the feature space could be named during the teaching using concepts and sub-concepts (Simard et al., 2017) the interpretable decision set could be used in a more easily interpretable fashion for example: "Exercise = Yes, Rugby=Yes then travel from A to B" 
or rephrased in natural language. Presenting predictions on a global level by selecting features of interest, from a calendar or map perspective, has parallels with work by Lakkaraju et al. (2019) in MUSE framework where features of interest can be selected to investigate predictions in sub-spaces of interest.

The TED (Hind et al., 2018) and MUSE (Lakkaraju et al., 2019) framework could be an interesting addition, implementing TED in our prototype would be beneficial in that meta-information in the form of concepts and sub-concepts can be added to labels corresponding to specific journeys. This would imply that interpretable information can, for example, be added for the concept "Exercise" that includes all journeys the commuter performs as sub-concepts to "Exercise". The concepts can then be used to inspect sub-spaces of interest in a form similar to MUSE. Our approach has similar goals concerning trust as Teso and Kersting (2019) but differs in that the agency in our case is closer to the human teacher that decides the extent of the knowledge that should be transferred.

Our work then suggests that through designing an explicable MT agent for a domain, over time the agent can be trusted. To reach this the agent has to be either, as in our case, matching a significant amount of predictions to the context in an explicable manner and/or support exploration regarding the model so the knowledge transferred can be assessed and understood.

\subsection{Conclusion}

This paper set out to explore how interpretability can be evaluated in an MT setting. Addressing evaluation of interpretability is an emerging research field (Doshi-Velez \& Kim, 2017; Boukhelifa et al., 2018), to this, our work adds a case study and evaluates interpretability as a design objective. One contribution is an increased focus on explicability as a relational quality of the ML agent. Explicability indicates that a domain expert can explain a prediction so it can be widely understood. How local and global explicability relates to the evaluation of interpretability are other contributions that can be important in many domains. Our work also shows how initial design goals concerning interpretability and explicability for an ML agent deeply influence the final result.

For future work, we suggest an increased focus on tools that support assessing a model's knowledge from an end user's, domain expert's and ML expert's perspective. Further research in this area can strike a balance between explicable and interpretable predictions and thus MT agents that 
evolve in incremental short cycles (Dudley \& Kristensson, 2018) in order to adapt to changes and support personalisation.

\section{References}

Biran, O., \& Cotton, C. (2017). Explanation and Justification in Machine Learning: A Survey. In IJCAI Workshop on Explainable AI (XAI) (Vol. 8, pp. 8-14).

Boukhelifa, N., Bezerianos, A., \& Lutton, E. (2018). Evaluation of Interactive Machine Learning Systems. In Human and Machine Learning (pp. 341-360).

Doshi-Velez, F., \& Kim, B. (2017). Towards a Rigorous Science of Interpretable Machine Learning. arXiv preprint arXiv:1702.08608.

Doshi-Velez, F., \& Kim, B. (2018). Considerations for Evaluation and Generalization in Interpretable Machine Learning. In Explainable and Interpretable Models in Computer Vision and Machine Learning. (pp. 3-17). Springer. doi: 10.1007/978-3-319-98131-4\{\\}1

Dudley, J. J., \& Kristensson, P. O. (2018). A review of user interface design for interactive machine learning. ACM Transactions on Interactive Intelligent Systems, 8(2). doi: 10.1145/3185517

Gilpin, L. H., Bau, D., Yuan, B. Z., Bajwa, A., Specter, M., \& Kagal, L. (2019). Explaining explanations: An overview of interpretability of machine learning. In Proceedings - 2018 IEEE 5th International Conference on Data Science and Advanced Analytics, DSAA 2018 (pp. 80-89). IEEE. doi: 10.1109/DSAA.2018.00018

Graneheim, U., \& Lundman, B. (2004). Qualitative content analysis in nursing research: concepts, procedures and measures to achieve trustworthiness. Nurse Education Today, 24(2), 105-112. doi: 10.1016/J.NEDT.2003.10 .001

Hind, M., Wei, D., Campbell, M., Codella, N. C. F., Dhurandhar, A., Mojsilović, A., ... Varshney, K. R. (2018). TED: Teaching AI to Explain its Decisions. Proceedings of the 2019 AAAI/ACM Conference on AI, Ethics, and Society, 123-129. doi: 10.1145/3306618.3314273

HLEG. (2019). Ethics Guidelines for Trustworthy AI (European Commission, 2019) (Tech. Rep.). High-Level Expert Group on Artificial Intelligence.

Hornik, K., Stinchcombe, M., \& White, H. (1989, 1). Multilayer feedforward networks are universal approximators. Neural Networks, 2(5), 359-366. doi: 10.1016/0893-6080(89)90020-8

Lakkaraju, H., Bach, S. H., \& Leskovec, J. (2016). Interpretable decision sets: A joint framework for description and prediction. In ACM SIGKDD International Conference on Knowledge Discovery and Data Mining (pp. 1675-1684). doi: 10.1145/2939672.2939874

Lakkaraju, H., Kamar, E., Caruana, R., \& Leskovec, J. (2019). Faithful and Customizable Explanations of Black Box Models. In Proceedings of the 2019 AAAI/ACM Conference on AI, Ethics, and Society (pp. 131-138) 
(pp. 131-138). Honolulu: ACM.

Lecun, Y., Bengio, Y., \& Hinton, G. (2015). Deep learning. Nature, 521(7553), 436-444. doi: 10.1038/nature14539

Lindvall, M., Molin, J., \& Löwgren, J. (2018). From machine learning to machine teaching. Interactions, 25(6), 52-57. doi: 10.1145/3282860

Lipton, Z. C. (2016). The Mythos of Model Interpretability. Communications of the ACM, 61(10), 35-43. doi: 10.1145/3233231

Lou, Y., Caruana, R., \& Gehrke, J. (2012). Intelligible models for classification and regression. In Proceedings of the ACM SIGKDD International Conference on Knowledge Discovery and Data Mining (pp. 150-158). doi: $10.1145 / 2339530.2339556$

Lundberg, S., \& Lee, S.-I. (2016). An unexpected unity among methods for interpreting model predictions. arXiv preprint arXiv:1611.07478.

Miller, T. (2019). Explanation in artificial intelligence: Insights from the social sciences. Artificial Intelligence, 267, 1-38. doi: 10.1016/j.artint.2018 .07 .007

Nielsen, L. (2013). Personas - User Focused Design. Springer. doi: 10.1007/ 978-1-4471-4084-9

Ribeiro, M. T., Singh, S., \& Guestrin, C. $(2016,2)$. "Why Should I Trust You?". In Proceedings of the 22nd ACM SIGKDD International Conference on Knowledge Discovery and Data Mining - KDD'16 (pp. 1135-1144). New York, New York, USA: ACM Press. doi: 10.1145/2939672.2939778

Simard, P. Y., Amershi, S., Chickering, D. M., Pelton, A. E., Ghorashi, S., Meek, C., ... Wernsing, J. (2017). Machine Teaching: A New Paradigm for Building Machine Learning Systems. arXiv preprint arXiv:1707.06742.

Teso, S., \& Kersting, K. (2019). Explanatory Interactive Machine Learning. In Proceedings of the 2019 AAAI/ACM Conference on AI, Ethics, and Society (pp. 239-245). ACM. doi: 10.1145/3306618.3314293 


\section{PAPER 2 - CONTEXTUAL MACHINE TEACHING}

Lars Holmberg, Paul Davidsson and Carl Magnus Olsson

\section{Abstract}

Machine learning research today is dominated by a technocentric perspective and in many cases disconnected from the users of the technology. The machine teaching paradigm instead shifts the focus from machine learning experts towards the domain experts and users of machine learning technology. This shift opens up for new perspectives on the current use of machine learning as well as new usage areas to explore. In this study, we apply and map existing machine teaching principles onto a contextual machine teaching implementation in a commuting setting. The aim is to highlight areas in machine teaching theory that requires more attention. The main contribution of this work is an increased focus on available features, the features space and the potential to transfer some of the domain expert's explanatory powers to the machine learning system. 



\section{Contextual Machine Teaching}

\subsection{Introduction}

Contemporary Machine Learning (ML) research is currently dominated by a technocentric perspective that primarily solves and addresses technical and functional goals but risks not placing enough focus on serving human values and ethical principles (IEEE, 2019). As an alternative, moving the agency towards domain experts and end users is an emerging and promising development in the field (Dourish, 2016; Bijker, 1993). A move in this direction can help democratize the knowledge and thus mitigate some of the risks of the problematic knowledge concentration of this disruptive technology (Zuboff, 2015; O'Neil, 2016; Couldry \& Mejias, 2019). An agency move in the direction of non-machine learning experts also offers the possibility to find and explore novel application areas that build on subjective knowledge production.

In Machine Teaching (MT) (Simard et al., 2017; Zhu et al., 2018), a human domain expert acts as a teacher in relation to a machine-based learner and consequently, domain knowledge becomes a natural part of the resulting deployment of an ML system. Previous studies mainly involve theoretical examples (Zhu et al., 2018; Chen et al., 2018; Liu et al., 2017; Mei \& Zhu, 2015) in the area of MT and the majority of the concrete studies are in the medical (Lindvall et al., 2018; Holzinger, 2016) and robotics field (Cakmak et al., 2010; Du et al., 2018; Peternel et al., 2015). Also, to our knowledge, no studies currently exist that explore MT in a personal knowledge domain that includes contextual data. Focusing an MT approach in this direction could open up for a new type of individualization and personalization in areas such as Personal Informatics, Assistive Technology, and Intelligent Personal Assistants.

Simard et al. (2017) see and treat MT as a discipline separate from traditional machine learning and have developed principles intended to be useful for MT implementations.

Our study is guided by a desire to understand what aspects of the MT paradigm (Simard et al., 2017) are the most important when it is applied on a contextual application in a personal knowledge domain. In this study 
we focus on answering the following sub-questions using a prototype we have developed for a commuting context:

1. What are the consequences, both in relation to the application and the MT paradigm, of applying the principles developed by Simard et al.?

2. What does a personal knowledge domain imply for the MT paradigm?

3. What does context dependence imply for the MT paradigm?

To answer the questions, we used the principles developed by Simard et al. (2017) as an analytic lens, a way of understanding the area and potentially strengthen the MT paradigm, in conjunction with a user study. The study spanned eight weeks, involved eight participants and one meeting every week.

In the remaining part of the work, we present the project background, outline our framing, our selection of MT principles and the methodological approach we applied. Thereafter we describe our prototype and the study setup. In the analysis and discussion, we relate MT principles to our prototype and identify areas where our work suggests that more research can bring the area forward. We end with a conclusion section where we elaborate on additional perspectives that needs further research.

\subsection{Related work}

Machine teaching differs from Active Learning (AL) (Settles, 2010) and Interactive Machine Learning (IML) (Fails \& Olsen, 2003) in that the teacher knows that, at some point in time, the extent of the knowledge they want to transfer to the learner (Simard et al., 2017). The focus in MT is on that predictions made by the machine learner has to be interpretable, for the teacher, in relation to the performed teaching. If the system is constructed with a specific domain in focus, the role of the ML expert can be reduced so a domain expert can own the process of refining the model's knowledge. The benefit of IML/MT systems in relation to more traditional ML systems "is observable in situations where the precise design objectives of the user are unclear and/or data labels cannot be obtained a priori" (Dudley \& Kristensson, 2018). MT and IML systems often facilitate short iteration loops where the model can be incrementally updated in small steps. Simard et al. states the role of the teacher as the person responsible for transferring knowledge using a teaching tool to the machine learner, in order to produce a model that can approximate a 
concept. Central to the teaching is the process when the teacher selects and labels examples that the teacher believes represents a concept.

Simard et al. (2017) propose the following principles for the domain specific language and the process of MT.

- The language should be universal over a domain and easy to learn. If they have the required domain knowledge, this results in the domain expert being interchangeable. The ML method becomes exchangeable, given that the MT language is independent of the ML method used.

- The teaching tool should be implemented is feature complete, implying that there are enough features for the teacher to separate all the desired target concepts. Feature completeness is seen as a responsibility of the tool, not the teacher.

According to Simard et al. (2017) the teaching process places demands on the sampling distribution, training distribution, and the deployment distribution. The sampling distribution, from which the examples are selected, must be rich and diverse so that the teacher can select a training distribution that generalize well on the deployment distribution.

\subsection{Methodology}

To explore and challenge contextual MT we applied the principles developed by Simard et al. (2017) as an analytical lens on a prototype. We chose Participatory Design (Robertson et al., 2012) as a frame for the research, as it allows for adaptations during the study in order to handle new insights. We recruited eight participants for a study that lasted eight weeks. The evaluation is an application grounded evaluation with real humans and real tasks (Doshi-Velez \& Kim, 2018).

\subsubsection{Research setting}

We selected commuting as our application area since it is accessible and easy to relate to. Commute patterns are individual and predictions are contextually dependent. We built a prototype giving a commuter control over the transfer of their commute pattern to the ML model. The MT interface of the prototype can be seen in Fig. 1 and the prediction part in Fig. 2.

We used the capabilities of modern mobile phones to collect contextual features(Schmidt, 1999), such as time, activity and location, used as con- 
text cues in order to make journey predictions and collect training data. The model is deployed without any initial data about the user's commute pattern and thus cold started in order to be trained using the teaching interface (Fig 1). In this work, we distinguish between different usage modes: in context teaching, out of context teaching, in context predicting and out of context predicting (We did not implement out of context prediction at this stage). In context teaching implies that at least one feature from the context cue is used. Out of context teaching implies that none of the features maps to the user's current context cue and therefor all desired features have to be manually selected. In context, prediction implies that all the features in the context cue are sent to the ML model so a prediction can be made and out of context prediction imply that parts of the context cue are simulated. The contextual features we selected as relevant for this prototype were location, day of week, time of day and the commuter's current activity (walking, still, running and in vehicle). A prediction is done when the app is started and the contextual features are sent to the trained model. The resulting prediction is then used to collect and present information about upcoming departures for the predicted journey (Fig. 2).

We used one individually trained Neural Network (NN) for each user as ML method. The choice of NN was primarily made since the selection simplifies prototyping in that $\mathrm{NN}$ handles raw input data and can handle multiple levels of representation (Lecun et al., 2015). For the implementation of the neural network, we used the framework from Fast.ai on a cloud server. A real-time database (Firebase), a NodeJS server and a Flask server was used for the machine learning pipeline and synchronization. Prediction turn around time is on average under one second and retraining time on average around 15 seconds. We used a network with two hidden layers that we feed with the contextual feature values: latitude (double), longitude (double), minute of day (integer minutes from midnight), weekday (integer one to seven) and activity (integer that represents walking, still, running and in vehicle). Weekday and activity are handled as categorical features and the other as continuous.

To handle the cold start situation the commuter selects a sub-space in the feature space consisting of feature ranges, for example, a time-span and one or more days like weekend using the teaching interface (Fig 1). We then generate synthetic examples inside the selected feature sub-space. To find reasonable prediction accuracy we performed a functionally grounded evaluation (Doshi-Velez \& Kim, 2018) with data created for three personas. 


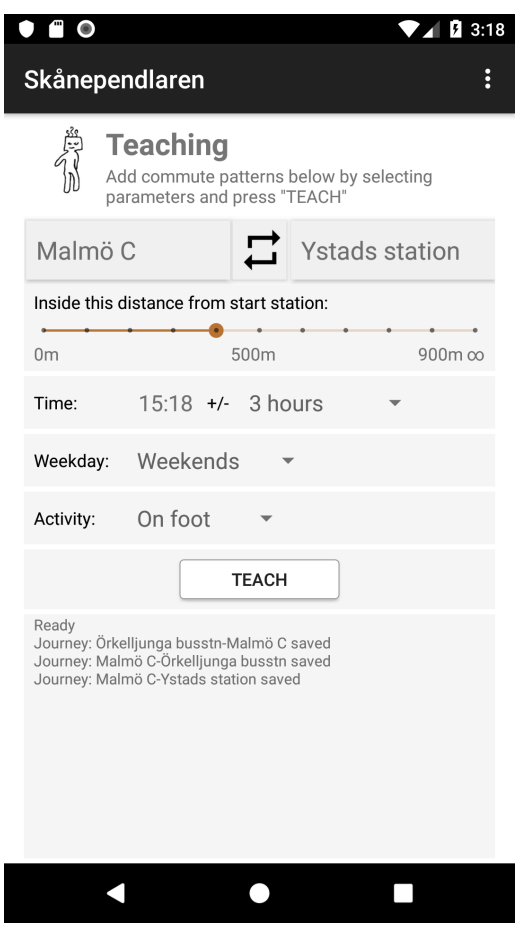

Figure 1: Teaching interface.

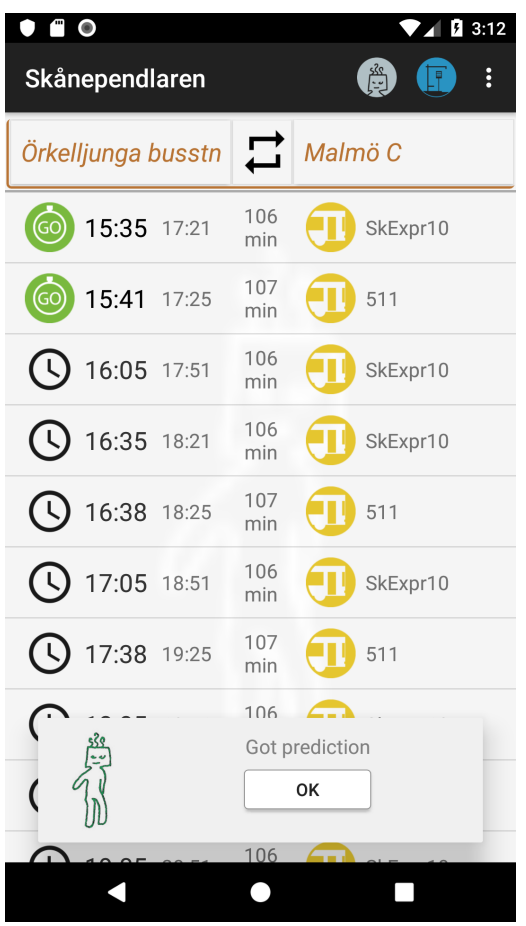

Figure 2: Prediction interface.

We iterated to find an appropriate size of the network (200|100 neurons), number of epochs (7) and a reasonable number of generated examples for a concept (40) to fulfill our prototype needs. We used an automatic learning rate finder (Smith, 2017) implemented in fast.ai to optimize the learning rate. The examples we generate are spread randomly using a uniform distribution over the feature sub-space that the user selects. For example, if you set weekday to "Any day" the examples are randomly spread over the days of the week. If you select "Friday" all examples have the value of 5 as training data. This is, of course, a simplification of the machine learning problem at hand but the goal at this stage was to build a working prototype for this study. Once we got the prediction performance acceptable, the back-end implementation (machine learning pipeline and model configuration) was frozen. This was done so we could get to a point where we could study and tweak our contextual MT implementation separated from an invariable machine learning back-end. 


\subsubsection{Recruiting participants for the study}

For the participatory design phase, we recruited users with a background in graphic design, interaction design, user experience design, programming or agile development. Our goal with this selection was the possibility to discuss and elaborate aspects of MT during the study with users who are accustomed to these types of discussions from both a technical perspective as well as a usage perspective. To recruit we mailed information about the project to some selected student groups and companies. Ten persons showed interest and we selected eight of them for the study. All the participants were under the age of 40 , four were female and four were male. Six out of eight participants were university students ranging from first-year bachelor to master's level in the range of between 20 and 30 years. One participant works in the industry as primarily UX designer and product owner (age 36) and one as a project manager (age 39). The two participants at master's level were international students. One requirement when recruiting users was that the users had to commute and own an Android phone. The users were rewarded with one month of free commuting during the study.

\subsubsection{Participatory generated data}

The study was conducted during eight weeks with weekly scripted recorded discussions with the participants. Initially, one to one meetings were held with the students to get to know them better. We then went to groups of two from a similar field and ended with groups of three or four with mixed competences. The intention with this was to create an atmosphere of creative discussion climate and interesting group constellations. The meetings started with a follow up on previous week's experiences where metrics and redesign proposals were presented and discussed. In addition, the following week's focus and tasks were discussed. Initially, the participants were introduced to the MT paradigm, and during the study, they evaluated different teaching strategies and gradually became accustomed to the paradigm and our prototype. In the later sessions, the participants were able to, in varying degrees, abstract the MT paradigm from the commuting context and thus give input that can be generalized to other domains. Using content analysis (Graneheim \& Lundman, 2004), interviews and workshops were broken down into codes originating from the MT vocabulary used by Simard et al. (2017). 


\subsection{Analysis and Result}

In this section, we use the MT principles developed by Simard et al. (2017) to better understand our contextual prototype and lift out aspects that come into focus. Those aspects in focus are further elaborated on their own or in conjunction with findings from our user study using the same prototype. The prototypes capabilities was summarized as follows by one study participant:

The first week we had this app I was quite impressed. [...] So I had only taught it that on "Mondays at this time and place I would like to travel from Malmö C to Svedala". Then on Tuesday when I opened the app it knew that this is the journey you would like to take. So it can know things that you didn't teach in detail, but only approximately.

\subsubsection{Teaching algorithm}

Our MT algorithm (Algorithm 1) differs at some key points from the process proposed by Simard et al.

- The user can teach a concept both in and out of the target context (the two while loops). This means that you can, for example, teach your complete commute pattern from home by manually selecting all features in Fig. 1 or you can, teach just before you take a transport by using the context cue.

-When teaching, examples are not selected from a sampling set but rather a sub-space in the feature space is defined in the teaching interface (Fig. 1) as belonging to a desired concept. The machine learning back-end then generates synthetic examples within this subspace.

- The user can only test towards a quality criterion when in the target context, in this case, this means to evaluate that the correct journey is predicted (the 'if-elseif-else' statement).

- Missing features cannot be added so feature blindness cannot be handled (the 'else-if' part) Fig. 1.

- If prediction errors occur due to inconsistent teaching, labeling errors, overlapping concepts or because the teacher distrust the predictions, the only alternative is to delete all training data and add all the desired concepts again (the 'else' part). 


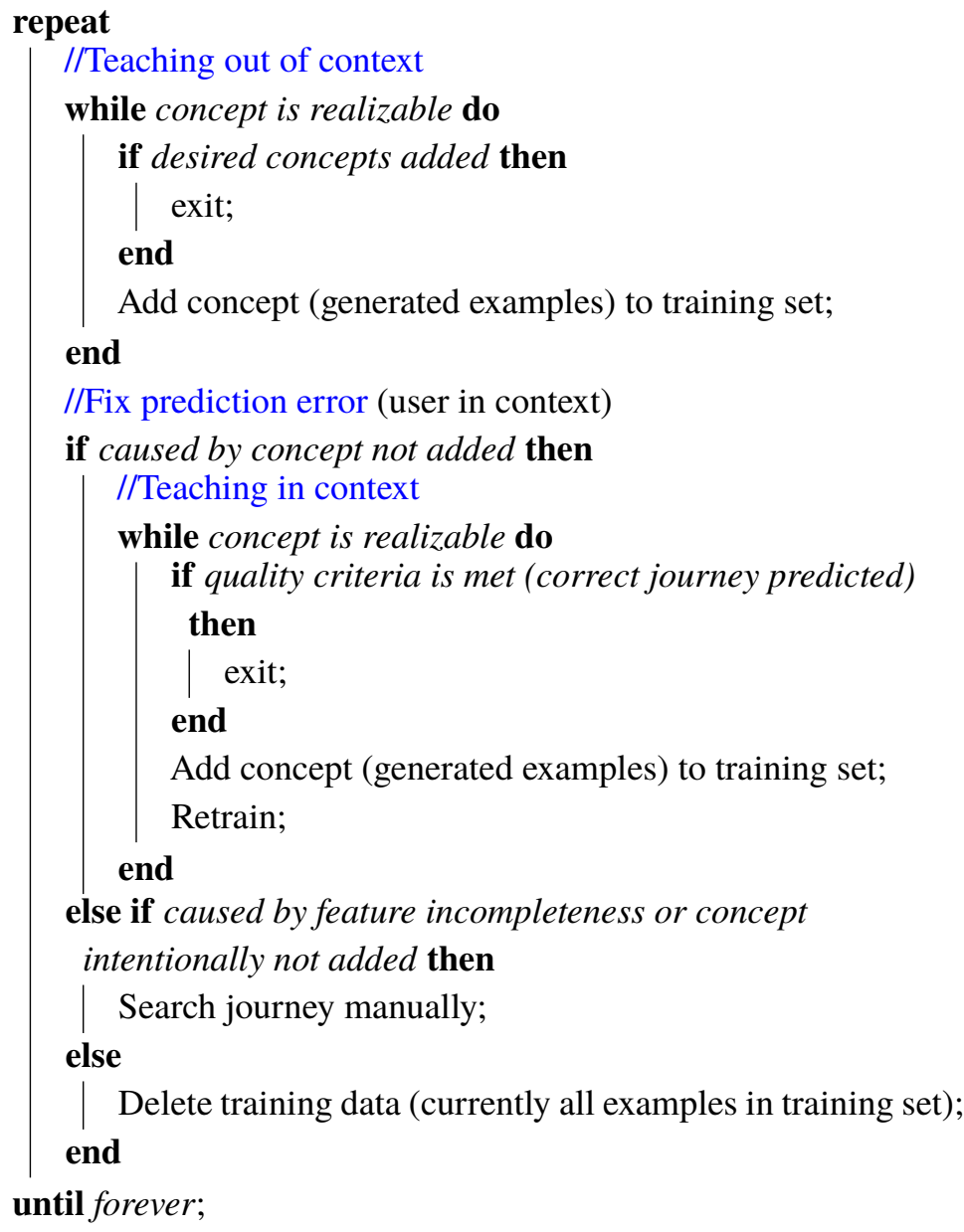

Algorithm 1: Contextual MT process

\subsubsection{Teaching strategies}

During week two of the study, the participants were instructed to do out of context teaching only once at the beginning of the week. During the third week, they did in context teaching when the prediction made was wrong compared to their intended journey. The prototype was reset prior to these tests. During the following weeks, the participants decided their own teaching strategies. Comment from one of the users on in context teaching compared to previous weeks out of context teaching:

It was a lot messier than last week. Eh.. harder to add things and you were more stressed and not that particular.. I had a lot 
less control over the things I added compared to last week.

In out of context teaching, the users tended to teach general concepts directly (using, for example, the selection "weekdays" instead of individual days). During in context teaching most participants changed some of the feature ranges to make the teaching more general, especially regarding time span, but only one made all teaching as general as possible. All users found the predictions interpretable in relation to what they had taught the artifact, even if the predictions as such were wrong in relation to their current context. It was harder to tell if the predictions were logical when they did in context teaching since the users found it difficult to remember if the whole concept had been taught or not. One of the users had complex commute patterns and experienced feature blindness (she works at two places and travels there depending on conditions that cannot be represented using the available features).

One user argued for why she, in theory, would prefer in context teaching, even if she, in this case, preferred out of context teaching:

In theory, I think I like in context teaching better since it is real events that occur and I only save them. But with the experience at the moment in the app, it feels better to add those teachings once. But that behavior could probably change if the app is changed.

A comment on combining the teaching strategies:

Can't you combine both? The first time you open the app as a new user you get an out of context teaching interface and add your most common journeys. And then when you done, you could teach it via a fast-teach-button, you get thumbs up/down in relation to a prediction.

\subsubsection{Prediction in and out of context}

In our implementation, the commuter can only receive predictions when in context. We are primarily interested if the teacher finds the in context predictions interpretable in relation to the performed teaching. The model's prediction accuracy becomes less interesting from a system level, as long as the prediction is correct, or as one participant expressed it:

It's more interesting for the developer, for you maybe. But not really for the user. I mean I just need the bus. 
The users with simple commuting patterns, for example to and from work and a few other non conflicting regular activities, found that the predictions were interpretable. When the teaching became complex or fragmented over time it was harder to conclude, from the commuter's perspective, if the predictions were logical in relation to the previously performed teaching.

Our study found that it is difficult to tell whether this was due to the choice of ML method, the examples created by the back-end, that the teacher was inconsistent in his/her teaching or whether the teacher forgot if a concept was completely taught. As one participant expressed it "I was more impressed in the beginning, but after a while, I think it got confused by all the teaching". Another comment was "there should be a way to check what it knows". In our implementation, when there is a mismatch between the expected predictions and the learned concepts, the only option is to completely delete the training distribution and reteach the complete commute concept. Our approach works well for the participants in the study who had simple commute patterns, so the complete commute pattern could be retaught out of context quickly and easily within a few minutes time or as an alternative retaught in context for each journey concept that appears as a context cue. The usual reaction from the participants is to reteach the intended journey and check the prediction. This situation is important because if the prediction is not corrected it is easy to mistrust the app. In any case, the ability to visualize the teaching done or probe predictions would be an important addition. As one participant expressed this:

But from the teacher's perspective when I teach him, can you show me what I teach him? ... So I know I am the teacher.

\subsubsection{Cold start}

In a personal knowledge domain, as we defined it in this study, no labeled or unlabeled examples exists initially, only an empty feature space limited by the available features and their ranges. Similar cold start challenges will likely appear for MT targeting the areas of Personal Informatics, Intelligent Personal Assistants and Assistive Technology. In these areas, it will likely be challenging to collect enough rich and diverse data in the background so a teacher can search and select examples representing all envisioned concepts. In context teaching and evaluation, as our study indicates, in an iterative fashion is then an interesting alternative approach. This does not rule out using previously collected data or using models transferred from 
similar domains or situations as a starting point.

\subsubsection{Transfer concept to learner}

The teacher's complete commute patterns are, on a top level, the concept they want to transfer to the learner. This top concept is for our context broken down by the teacher to mutually exclusive sub-concepts in a tree structure that, depending on the teacher, could be, for example, on the lowest level, "Traveling from home to work" or "Going home from Sara". In our case, the teacher can readily express those concepts in terms of the available features. This decomposition and translation to feature ranges is, in our case, done using an implicit schema in the teacher's head and is not represented or saved in the application. If the knowledge domain were public or shared, many concepts would be part of the domain specific vocabulary for that domain. In our personal case there could be pre-created concept names like "Going to work" etc. and/or a possibility to name mundane personal concepts. The possibility to name and use concepts during teaching was not requested by the study participants, but it stands out as a result of this study that would be interesting to study further and as an important part of a teaching language.

\subsubsection{Teaching Language}

In this study, we created a domain specific MT language that can aspire to be universal across the commuting domain. By using our language, a commuter can teach a learner his/her commute patterns as long as an application is connected to a relevant service from a transport provider.

Limitations in our language implementation stem from the fact that our implementation is not feature complete and we cannot add missing features. For our prototype, we adopted a weaker alternative approach to feature completeness in that we allow users to ignore existing features, to make it clear that, for example, a journey prediction is only dependent on location and not time, day and activity. To make this more explicit for our study participants in order for, feature completeness to be discussed in our study, we made this option clearer in a later iteration of the prototype. All our study participants found location, day of the week, and time to be relevant and useful features but none of the participants found activity (still, walking, running or in vehicle) to be a useful feature. The teachers in our case have access to the complete feature space and this gives them the possibility to teach a concept in any part of this space. In comparison, the 
principles by Simard et al. (2017) only gives access to the feature space through existing examples in the sampling distribution, this then has to be rich and diverse in order to offer a possibility to teach all intended concepts.

In relation to those principles, we have pre-created the composed features weekdays and weekends so the user can select them directly. In contrast to the principles developed by Simard et al. (2017) we do not give the users a possibility to define, name and compose new features for example "Mornings", "Mornings on weekends at Helens's place" or "Night". Instead, teaching must be carried out a number of times for one concept instead of reusing created composed features. Additional features suggested and discussed by our study participants were personal calendar, travel time, preferred means of transport and weather data.

\subsection{Discussion}

In this section, we outline and compare our results and analysis, with related MT theory and practice.

At the heart of MT lays the teacher's domain knowledge and consequently the capability to map an example with a label representing a concept. In our case, when the app is first started, no labeled or unlabeled examples exist and the commuter has no knowledge of the ML method the learner's uses. In the work by Simard et al. (2017), there are examples representing the concepts to choose from in a sampling distribution. In our case, the lack of examples is compensated by the teacher using their domain knowledge to select a sub-space in the feature space that represents a concept. Our approach is a direct result of treating the domain as a personal knowledge domain that uses a limited number of human interpretable features and that no examples have been collected by the user prior to the initial teaching of a concept. Our approach emphasizes the construction of a learner that, in relation to the concepts taught and the commuter's current context, includes an ML method that gives predictions that are interpretable. This in order for the teacher to be able to, over time, create a cognitive model over how to teach the model.

MT systems must take into account that the concepts and the schemes that connect them are not stable over time in relation to the teacher and in relation to the world. These are challenges that we have not addressed in this work. We did also not save any training data during use, making use of this data could be one possible route, from an ML perspective, to fine-tune 
the system and address concept- and covariant- shift (Moreno-Torres et al., 2012). Combing a domain specific taxonomy and a possibility to name mundane concept with the possibility to rearrange concept data seems like a useful approach, from a teacher's perspective, to address dataset shift. This possibility to rearrange, add and delete concept also opens up for a domain expert to address their knowledge development and shifting usage needs within the domain.

Traditional programming languages often aim to be oblivious about the usage domain and more specifically in relation to the intended technical platform or type of problem it can solve. In contrast, teaching languages are specific to the usage domain and closely connected to the vocabulary of that domain. Some features, like time and location, will be represented and needed in many contextual teaching languages given that they are used in many domains. Central to a teaching language for any domain is the available features that the language gives access to. Here, as Simard et al. (2017) and our work points out, it is central that features can be created, added, removed, composed and that sub-spaces in the feature space can be ignored. In our case, when the knowledge domain is personal, the need for a flexible teaching language is central, but will probably not cover all individual cases. From our case, the weather situation could be relatively straight-forward to add, at least for in context teaching and in context predictions, but integration with a personal calendar is a complex feature expansion that needs special attention.

\subsection{Conclusions}

We set out to apply principles for MT (Simard et al., 2017) onto a prototype with the goal of gaining a better practical understanding of the MT paradigm in a contextually dependent setting. We found, in relation to our first research question, that our selected MT principles make it possible to separate and discuss MT as a discipline in its own right and that a domain expert could apply, teach, discuss and evaluate a learner independent of the machine learning expert. In relation to our second research question, we found that MT can be a fruitful and promising approach to make machine learning useful in supporting personal and individual needs, useful for example in the area of intelligent personal assistants. Regarding the third research question, we identified areas of the MT paradigm that needs further research to be applicable in a contextually dependent domain; in context teaching, teaching over time, possibilities to probe the 
learner for learned concepts and interpretations of predictions given. In terms of future research, we suggest a direction and a focus on integrating the explanatory powers of humans as a natural part in machine learning systems via an MT approach with an aim towards building the systems we want and not solely the one we can.

\subsection{Acknowledgment}

I would like to thank Jonas Löwgren for invaluable support during the work.

\section{References}

Bijker, W. E. (1993). Do Not Despair: There Is Life after Constructivism. Science Technology, \& Human Values Sage Periodicals Press, 18(114), 113-138.

Cakmak, M., Chao, C., \& Thomaz, A. L. (2010). Designing Interactions for Robot Active Learners. Computing in Science \& Engineering, 2(2), 108-118.

Chen, Y., Singla, A., Mac Aodha, O., Perona, P., \& Yue, Y. (2018). Understanding the Role of Adaptivity in Machine Teaching: The Case of Version Space Learners. Advances in Neural Information Processing Systems, 1476-1486.

Couldry, N., \& Mejias, U. A. (2019). Data Colonialism: Rethinking Big Data's Relation to the Contemporary Subject. Television \& New Media, 20(4), 336-349. doi: 10.1177/1527476418796632

Doshi-Velez, F., \& Kim, B. (2018). Considerations for Evaluation and Generalization in Interpretable Machine Learning. In Explainable and Interpretable Models in Computer Vision and Machine Learning. (pp. 3-17). Springer. doi: 10.1007/978-3-319-98131-4\{\_\}1

Dourish, P. (2016). Algorithms and their others: Algorithmic culture in context. Big Data \& Society, 3(2), 205395171666512. doi: 10.1177/ 2053951716665128

Du, G., Chen, M., Liu, C., Zhang, B., \& Zhang, P. (2018). Online robot teaching with natural human-robot interaction. IEEE Transactions on Industrial Electronics, 65(12), 9571-9581. doi: 10.1109/TIE.2018.2823667

Dudley, J. J., \& Kristensson, P. O. (2018). A review of user interface design for interactive machine learning. ACM Transactions on Interactive Intelligent Systems, 8(2). doi: 10.1145/3185517

Fails, J. A., \& Olsen, D. R. (2003). Interactive Machine Learning. In Proceedings of the 8th International Conference on Intelligent User Interfaces (pp. 39-45). ACM. doi: 10.1145/604045.604056

Graneheim, U., \& Lundman, B. (2004). Qualitative content analysis in nursing research: concepts, procedures and measures to achieve trustworthiness. Nurse Education Today, 24(2), 105-112. doi: 10.1016/J.NEDT.2003.10 
.001

Holzinger, A. (2016). Interactive machine learning for health informatics: when do we need the human-in-the-loop? Brain Informatics, 3(2), 119-131. doi: 10.1007/s40708-016-0042-6

IEEE. (2019). The IEEE Global Initiative on Ethics of Autonomous and Intelligent Systems. Ethically Aligned Design: A Vision for Prioritizing Human Well-being with Autonomous and Intelligent Systems, First Edition. , 291.

Lecun, Y., Bengio, Y., \& Hinton, G. (2015). Deep learning. Nature, 521(7553), 436-444. doi: 10.1038/nature14539

Lindvall, M., Molin, J., \& Löwgren, J. (2018). From machine learning to machine teaching. Interactions, 25(6), 52-57. doi: 10.1145/3282860

Liu, W., Dai, B., Humayun, A., Tay, C., Yu, C., Smith, L. B., ... Song, L. (2017). Iterative Machine Teaching. Proceedings of the 34th International Conference on Machine Learning (ICML), 2149-2158.

Mei, S., \& Zhu, X. (2015). Using Machine Teaching to Identify Optimal Training-Set Attacks on Machine Learners. Twenty-Ninth AAAI Conference on Artificial Intelligence, 2871-2877.

Moreno-Torres, J. G., Raeder, T., Alaiz-Rodríguez, R., Chawla, N. V., \& Herrera, F. (2012). A unifying view on dataset shift in classification. Pattern Recognition, 45(1), 521-530. doi: 10.1016/j.patcog.2011.06.019

O'Neil, C. (2016). Weapons of math destruction: How big data increases inequality and threatens democracy. Broadway Books.

Peternel, L., Petrič, T., \& Babič, J. (2015). Human-in-the-loop approach for teaching robot assembly tasks using impedance control interface. Proceedings - IEEE International Conference on Robotics and Automation, 2015-June(June), 1497-1502. doi: 10.1109/ICRA.2015.7139387

Robertson, Toni, Simonsen, \& Jesper. (2012). Routledge International Handbook of Participatory Design. Routledge.

Schmidt, A. (1999). Advanced Interaction in Context. First Symposium on Handheld and Ubiquitous Computing (HUC '99), 89-101.

Settles, B. (2010). Active Learning Literature Survey (Tech. Rep. No. January). University of Wisconsin-Madison.

Simard, P. Y., Amershi, S., Chickering, D. M., Pelton, A. E., Ghorashi, S., Meek, C., ... Wernsing, J. (2017). Machine Teaching: A New Paradigm for Building Machine Learning Systems. arXiv preprint arXiv:1707.06742.

Smith, L. N. (2017). Cyclical Learning Rates for Training Neural Networks. In 2017 IEEE Winter Conference on Applications of Computer Vision (WACV) (pp. 464-472). IEEE.

Zhu, X., Singla, A., Zilles, S., \& Rafferty, A. N. (2018). An Overview of Machine Teaching. arXiv preprint arXiv:1801.05927.

Zuboff, S. (2015). Big other: Surveillance capitalism and the prospects of an information civilization. Journal of Information Technology, 30(1), 75-89. doi: $10.1057 /$ jit.2015.5 



\title{
PAPER 3 - THE ROLE OF EXPLANATIONS IN HUMAN-MACHINE LEARNING
}

\author{
Lars Holmberg, Stefan Generalao and Adam Hermansson
}

\section{Abstract}

In this paper, we study explanations in a setting where human capabilities are in parity with Machine Learning (ML) capabilities. If an ML system is to be trusted in this situation, limitations in the trained ML model's abilities have to be exposed to the end-user. A majority of current approaches focus on the task of creating explanations for a proposed decision, but less attention is given to the equally important task of exposing limitations in the ML model's capabilities, limitations that in turn affect the validity of created explanations. Using a small scale design experiment we compare human explanations with explanations created by an ML system. This paper explores and presents how the structure and terminology of scientific explanations can expose limitations in the ML model's knowledge and be used as an approach for research and design in the area of explainable artificial intelligence. 



\section{The Role of \\ Explanations \\ in Human-Machine Learning}

\subsection{Introduction}

Machine Learning (ML) and Artificial Intelligence (AI) systems are increasingly competitive to humans when it comes to decisions ${ }^{1}$ within a well-defined domain (Chollet, 2019). However, these increasingly complex systems often struggle to present causes that underpin a proposed decision as well as communicating their limitations in manners and forms that human users of the systems can understand (Marcus, 2018; Lipton, 2016; Doshi-Velez \& Kim, 2017).

There is a growing research interest focused on explaining these blackboxed decisions by summarising reasons for a decision (Lakkaraju et al., 2019; Lundberg \& Lee, 2017; Ribeiro et al., 2016; Selvaraju et al., 2020; Akula et al., 2020; Bengio et al., 2013; Ghorbani et al., 2019; GonzalezGarcia et al., 2018). A large quantity of the current work focus on creating causal attributions related to the trained ML model as such, and less focus is on supporting user's understanding of the ML system's capabilities (Miller, 2019; Lu et al., 2019). In this article, we address parts of this challenge by focusing on how ML system's limitations can be communicated to designers and end-users by using scientific explanations as an analytic lens. Using this lens we compare human explanations and explanations for a decision created by an ML system (Lipton, 2016). Scientific explanations are used since they are a form of explanations that uses observations to explain something we see in the natural world (Woodward, 2019; Hempel \& Oppenheim, 1948; Overton, 2012). We also consider scientific explanations as useful since they are, in line with mundane explanations, initiated by a why-question but, contrary to many forms of human explanations, adds rigour to the answer. This rigour can then function as a designer goal, facilitating the creation of trustworthy ML systems. The goal of this work is to add new perspectives related to limitations of ML systems in the area

\footnotetext{
${ }^{1}$ We use the word 'decision' throughout this paper to refer to the output from ML system in the form of classifications, recommendations, predictions, proposed decisions or actions.
} 
of eXplainable Artificial Intelligence (XAI) (Barredo Arrieta et al., 2020; Guidotti et al., 2018; Biran \& Cotton, 2017; Gilpin et al., 2019; Adadi \& Berrada, 2018; Hoffman et al., 2020).

To address this challenge we performed a design experiment in an accessible public domain (hand-painted porcelain). In this setting, both the ML system and a human can lift out evidence for the decision, that to some extent overlap. This opens up the possibility to compare the evidence a human lift out with those emphasised by the ML system. We carefully selected our design experiment so a layperson can relate to the domain while it still contains a complexity that can elicit questions and discussions. Discussions we open up for are design considerations transferable and generative for diverse domains as, for example, medical applications (Lindvall et al., 2018; Kim et al., 2018; Wang et al., 2019) and evolutionary biology (Ferreira et al., 2020). In this study, we are then guided by this research question:

- How can scientific explanations be used to better understand an ML system's limitations?

Answering this question is important when the goal is to design ML systems that function as a tool that can promote human understanding. In our design experiment, we selected a use context that builds on similar physical objects. We chose this setting since the notion of similarity (Medin et al., 1993) opens up the research perspective we aim to investigate, a situation where more than one equally valid explanation for a decision can exist. Answers related to a why-question, formulated as scientific explanations, can in this setting be compared to human explanations and explanations for a decision created by an ML system. From a technological perspective, we delimit our work by focusing on inductive black-box learners in the form of neural networks and saliency maps that emphasises, using some type of visual image overlay, areas important to a promoted decision. Here we build on the capability of neural networks to, based on images as input data, create internal knowledge representations (Lecun et al., 2015) and the capability of saliency maps to visualise some of these representations (Selvaraju et al., 2020).

To shed light on our research question, we situate our research focus in Section 1.2. On this follows our methodological approach outlined in Section 1.3, where we describe our research setting. Result and analysis follow in Section 1.4. Thereafter, in Section 1.5, we discuss consequences related to our research results. Our conclusions can then be found in the 
final section, Section 1.6.

\subsection{Related Work}

AI systems are increasingly used to promote decisions influencing our lives, consequently, it is important that decisions that originate from AI systems are trustworthy (HLEG, 2019). Extensive and active research has been conducted in the area of trustworthy AI (Biran \& Cotton, 2017; Gilpin et al., 2019; Lipton, 2016; Lakkaraju et al., 2019; Lundberg \& Lee, 2017; Ribeiro et al., 2016; Gunning, 2017; Ribera \& Lapedriza, 2019; Kulesza et al., 2015; Abdul et al., 2018). Surveys of methods for explaining blackbox models found a lack of human focus in the area (Doshi-Velez \& Kim, 2017; Lipton, 2016; Miller, 2019; Guidotti et al., 2018; Adadi \& Berrada, 2018). In this work, we focus on limitations of explanations related to a singular post hoc decision as a path to better understand the ML system as such (Ribeiro et al., 2016). Concerning explainability and interpretability, we, in line with Gilpin et al. (2019): 'take the stance that interpretability alone is insufficient. For humans to trust black-box methods, we need explainability -models that can summarise the reasons for neural network behaviour, gain the trust of users, or produce insights about the causes of their decisions.' Our work focuses on producing insights concerning the causes and validity of a decision.

The complexity of explanations in a human context is multifaceted even if we, as in this study, delimit us to causal explanations related to a why question. To approach the area we distinguish between causal attribution and causal explanation since, in the attribution case, no interpersonal exchange take place (Hilton, 1990). Causal attribution is consequently more closely related to the notion of interpretability, which often is the focus in XAI research (Miller, 2019). Causal attribution in an ML context then implies presenting how, using reasons, input data relates to a proposed ML decision. A causal explanation for an ML context implies an exchange with the goal of a human explainee's understanding. An explainer, in our case represented by the ML system, is then the part proposing an explanation and an explainee a human that tries to understand the explanation.

To summarise: causal attribution implies a decontextualised focus on the ML model by ascribing a phenomenon to its origin by connecting input data to a promoted decision whilst causal explanations have a wider goal in that they aim to create understanding related to the decision for a human explainee. Understanding then includes insights concerning the difference 
between the reality and the part of the reality modelled by the ML system. By understanding this a human explainee can to a larger degree judge the validity an explanation in relation to a specific use context. In a human context, this translates to being able to understand the knowledge level of the explainer, for example, we assess the answer differently to the question of why the sun rise in the morning if an explanation originates from a six-year-old or a physics professor.

One route to partly address this is to apply some restrictions concerning the form and type of explanations we aim to analyse, both those originating from a human and those originating from an ML model. Scientific explanations (Woodward, 2019) is then close at hand, since they span many disciplines, are well-known and, most importantly, initiated by a mundane why-question similar to a common type of explanations used in non-scientific settings. We can then use the terminology of DeductiveNomological (D-N) explanations (Hempel \& Oppenheim, 1948) implying that asking a why-question opens up an explanandum, a space that consists of explanans that, somewhat simplified, can be equalled to all possible statements that can be used to build an explanation related to the why-question. These explanans consists of zero to $n$ statements of the type: if $\mathbf{X}$ then $\mathbf{Y}$ and at least one law. Definition of what constitutes a law is disputed but can in this work be defined as an, for the domain, exceptionless generalisation (Woodward, 2019). In this work we couple causal attributions, evidence underpinning a decision, and statements in the D-N model.

Explanations, often implicitly, bring forward not only why a specific event $\mathbf{P}$ happened but also why $\mathbf{P}$ happened instead of event $\mathbf{Q}$ (P. Lipton, 1990). Answering a why question then corresponds to a causal explanation whilst a contrastive explanation corresponds to answering a why-not question. In this work, we do not discuss counterfactual explanations that answer to a what-if question, which demands imagining alternative scenarios, an area that is not part of our experimental study.

Overton $(2013,2012)$ used, among other methods, text mining on 781 articles in the journal Science across a wide range of disciplines to find categories common in scientific explanations. Overton's model can be seen in Figure 1. It consists of five categories of properties/objects applicable to explanations in science:

- Theories: sets of principles that form building blocks for models.

- Models: an abstraction of a theory that represents the relationships between kinds and their attributes. 
- Kinds: an abstract, universal class that supports counterfactual reasoning.

- Entities: an instantiation of a kind.

- Data: statements about activities (e.g. measurements, observations).

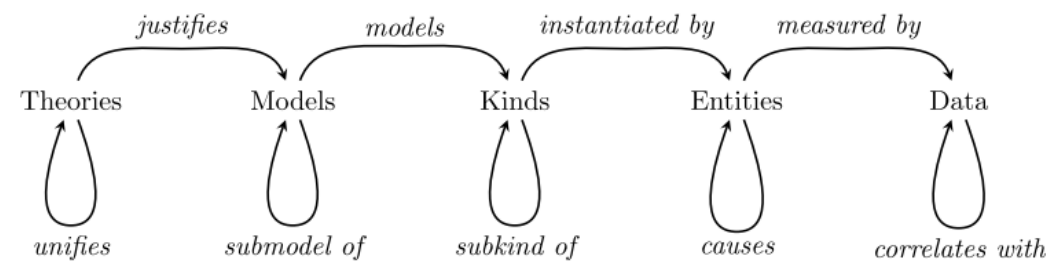

Figure 1: Overton's five categories and four relations in scientific explanation reproduced with permission from Overton.

This model supports causal explanations that build on contrastive and counterfactual reasoning. An explanation at one categorical level, must, in this model be relative to, and refer to, at least one other level to be causal and explanations between two levels must refer to all intermediate levels. We will use this model to better understand and compare explanations created by the ML-based explainer with explanations created by humans.

Entities are a special category, in relation to causality, since they are concrete particulars and exists in time and space. They are created, endure for some time and are then destroyed. Entities, as a result of their concrete existence, connect to data through one or more causal attributes forming causal chains.

We focus, as discussed above, on a situation where the ML-based learner creates post hoc causal attributions related to an image classification problem. In this type of setting causal attributes can be organised using higherlevel human concepts (semantic representations), for example 'narrow lines', 'beaklike', 'hairy', 'blue' or 'snow-white'.

A simple definition of concepts is hard to do (Genone \& Lombrozo, 2012; Murphy, 2004), and in this work we define them in line with Ghorbani et al. (2019) in that they need to be, in relation to the domain, meaningful, coherent and important. In the field of object classification in images, it is common to focus on kinds, timeless instances, for example, animal species, tables or chairs. Entities, on the other hand, are created, changed and destroyed, a valid explanation has a temporal dependence and 
is, to some extent, related to the notion of similarity (Medin et al., 1993; Gentner \& Markman, 1997).

In this work we focus on entities, instead of kinds, by doing this we create an explanandum where human capabilities and causal attributions proposed as explanations by an ML model overlaps in the form of lowlevel concepts related to entities. By this focus on entities we open up an explanandum where users without specific domain knowledge can use lower-level concepts, for example, 'colours' and 'shapes' to create an explanation. This is the setting we aim for in this work, a setting that can contain many parallel and equally valid explanations for a classification.

In ML there exists several methods both for lifting out causal attributes (Ribeiro et al., 2016; Selvaraju et al., 2020; Lundberg \& Lee, 2017; Lakkaraju et al., 2019) and work that aims to name and identify these attributes as concepts using previously defined concepts (Ghorbani et al., 2019; Amershi et al., 2009; Koh et al., 2020; Gonzalez-Garcia et al., 2018; Kornblith et al., 2019; Samek et al., 2020; Bengio et al., 2013; Akula et al., 2020). There exists, to our knowledge less work that focuses on finding and naming concepts during usage (Ghorbani et al., 2019). In this work we focus on saliency maps (Ribeiro et al., 2016), a method that marks areas in an image that corresponds to the causal attributes the ML models uses as evidence for a decision.

\subsection{Method}

In our study we aimed for a setting where humans and an ML model share parts of the explanandum, resulting in a situation where we can compare evidence for a decision that originates from humans and ML systems. We can then apply our theoretical approach based on scientific explanations, as outlined in Section 1.2, and investigate if that approach can be generative and useful to discuss ML system limitations productively. To make the study feasible we aimed for a small and focused design experiment where causal explanations, from humans, and causal attributions, from an ML system, can be collected and discussed in a controlled manner.

\subsubsection{Research Setting}

The six porcelain plates shown in Figure 2 is our selected setup for this study. They are hand-painted coffee plates from the Spisa Ribb series by the Swedish porcelain factory, Gustavsberg. Spisa Ribb features bone china with a transparent glaze, a brown edge and black vertical lines. The 
six plates used in this study were produced before 1974 when the plates were still painted by hand.

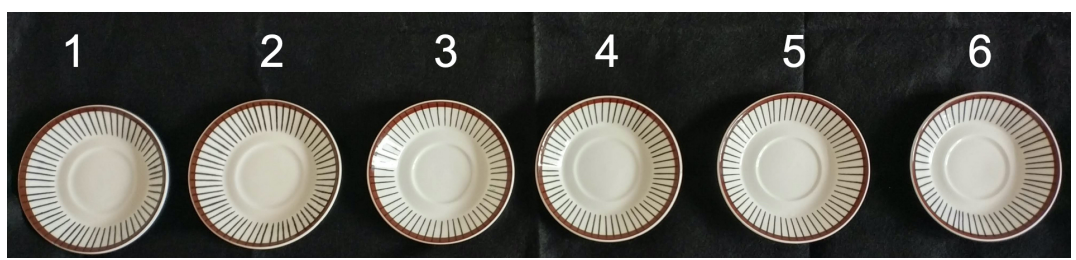

Figure 2: Spisa Ribb hand-painted plates

To the naked eye, certain characteristic attributes of the plates distinguish one plate from another. These attributes can take the form of irregularities in the painting, such as the length of the lines, the thickness of the lines, the distance between the lines and stains of paint on various areas of a plate.

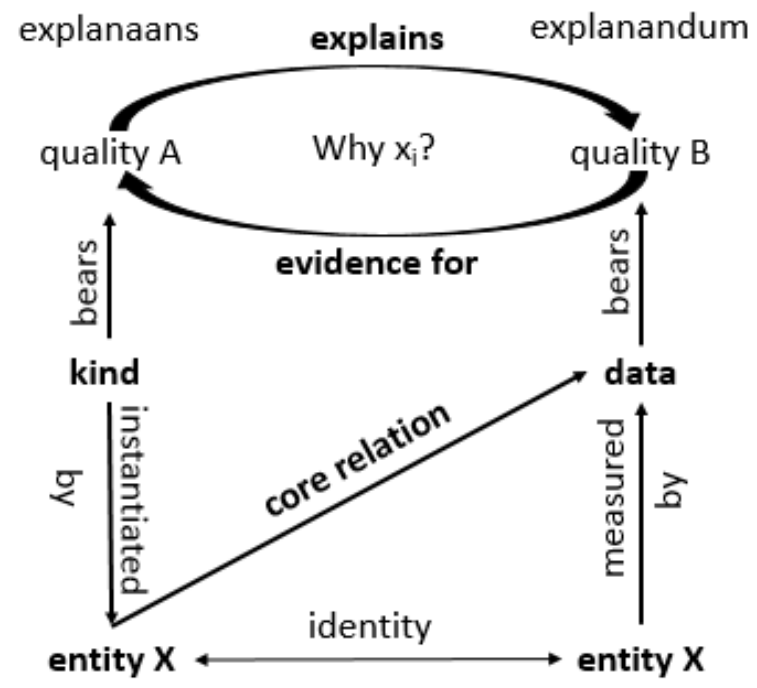

Figure 3: Structure for scientific explanations in our experiment with core relation between plates $\mathrm{X}$ and data in the form of images picturing the plates.

Using Overton's model (See Section 1.2) outlined in Figure 3. Our plate collection $X=\{1 . .6\}$ corresponds to entities that are instantiations of a kind i.e. the selected type of hand-painted plates (Spisa Ribb). The core relation used for classification is then, for the ML system, between the collection of plates $\mathrm{X}$ and the data represented by the images used for training. The data in the form of images used for training bears the qualities that constitute the 
set of qualities B used to identify the plates. These qualities correspond to internal knowledge representations created in the ML model during training. The qualities B are then causal and contrastive attributions that can be used as evidence for a classification. The explanandum for a specific decision promoted by the ML model is created by a why question, perhaps implicitly, posed by the human explainee. For example in a causal case why is this plate 6 , or in a contrastive case why is this plate 6 and not plate 4 . Explanans/causal attributions can then be selected from the explanandum as evidence for a decision and used to create an explanation that explains the promoted decision. A quality in set B could be an area in an image that corresponds to the concept 'paint-stain', a quality in set A that the kind (Spisa Ribb) bears.

Distinguishing the individual plates in our collections from each other poses a challenge for humans, but under ideal circumstances, a trained Convolutional Neural Network (henceforth CNN) can repeatedly identify them in images with $100 \%$ accuracy. For our experiment, we used a ResNet-50 model pretrained on ImageNet ${ }^{2}$ and an ML framework from Fast.ai. By using a pretrained model the neural network can use previously learned internal representation that can correspond to, in a human sense, lower-level concepts like 'colours', 'circles', 'corners', et cetera and combine them to represent higher-level internal representations brought by the new target domain. This significantly reduces the amount of training data needed to retrain the model and adapting it to the target domain. Our training data consisted of 48 images/plate, the validation set of 16 images/plate, all images used were of the size 640x640px.

We trained our CNN to reach high accuracy so it could classify the validation set without errors. This was done since our goal was not classification metrics, but rather, to use the trained ML model to create good-quality saliency maps that can present causal attributions for the test participants. We selected six images from the validation set (one for each plate) and used a saliency map implementation called Grad-CAM based on Selvaraju et al. (2020) implemented in the framework from fast.ai. ${ }^{3}$ The images used and the saliency maps can be seen in Figure 4. Examples of details that the saliency maps highlight can be seen in Figure 5 and Figure 6.4

After discussions and unstructured experiments within the research team, we decided to use six plates for our experiment. With that num-

\footnotetext{
${ }^{2} \mathrm{http}: / /$ www.image-net.org/

${ }^{3}$ https://docs.fast.ai/vision.learner.html\#_cl_int_plot_top_losses

${ }^{4}$ https://github.com/k3larra/spisaribb
} 
ber, we expected that a layperson, with some limited training, could learn to confidently separate them. If fewer plates were selected, we expected less developed explanations, and with more plates, we assumed that the experiment would be too hard and tedious and the explanations hard to remember and connect to a specific plate.

\subsubsection{Study Design}

We designed a study consisting of: a controlled experiment (Ko et al., 2013) followed by a semi-structured interview (Myers \& Newman, 2007). In the controlled experiment (Phase 1 and Phase 2) we perform training and evaluate the participant's ability to separate the plates. In Phase 2 we present half of the participants to the saliency maps. Finally, one interview session followed Phase 3.

We selected a small demographically narrow group of eight newly examined bachelor's students from the University as our study participants.

Phase 1: This phase was split into three parts, one learning part and two test parts:

- In the learning part, the participants studied the physical plates for a maximum of 5 minutes. The task assigned to the participants was to memorise and connect a predefined number from one to six to each plate.

- In the first test phase, the test manager presented one plate at a time for the participants. The task for the participant was to identify a plate by its number (See Figure 2). The six plates were presented three times each in a pseudo-random order.

- In the second test phase, all six plates were placed in front of the participants. The task for the participant was to identify them using their number (See Figure 2).

Phase 2: This phase was identical to Phase 1 except that, during the learning part, Participants 5-8 had access to saliency map explanations The saliency maps were presented on one paper per plate, showing the number of the plate and two images, one picturing the plate and one picturing the same plate with saliency map overlay (scale 1:1). These images are identical to those shown in Fig. 4.

Phase 3: Interview Our focus for the interview session was to better understand the intersection between human explanations and evidence for 
a decision that an ML system can produce. With reference to the top part of Figure 3 we are interested in finding common ground for quality A and quality B so the explanandum lifted out by the ML system can work in tandem with the user's understanding related to an answer to a whyquestion. The goal is to distil statements that can build explanations using scientific explanations as a format.

Using content analysis we identified and studied the statements the study participants created to explain how they separated the plates (Graneheim $\&$ Lundman, 2004). The goal of Phase 1 and 2 were then both to find evidence that the explanandum is shared but also to enforce study participants to build knowledge useful to create explanations. We directed our main discussion during the interviews toward the participant's memory strategy, which resulted in that they verbalised explanations using their own formulations. Also, we discussed the saliency maps to understand if they could be useful for the explainee's understanding and underpin their ability to produce explanations.

\subsubsection{Data Collection}

The interview started with the following instruction, and if exposed to the saliency map, one question:

- Describe your memory strategy.

- If you studied with the Grad-CAM explanations (saliency maps), did you find them useful?

The study was performed individually as one session that took around 30 minutes. The participants were not informed of their results at any stage. The study was performed by two researchers that followed a script developed during rehearsal sessions to ensure that the study was performed in the same way for all participants. The researchers took notes during the interview as documentation.

\subsubsection{Data Analysis}

Metrics related to Phase 1 and Phase 2 are presented in more detail under Section 1.4 together with the results. From the interview material, we searched for categories that mapped to statements that were used by the participants to separate the plates. Concepts (See Section 1.2) was identified to be the mutually exclusive and exhaustive categories the study participants used to separate the plates. Examples of concepts are for ex- 
ample 'Paint stain' and 'Line blurred'. By analysing the content related to the interviews with the participants exposed to the saliency maps we could also relate the concepts to the saliency maps.

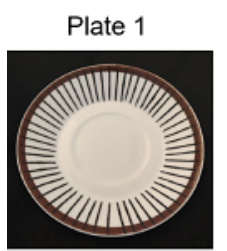

Plate 2

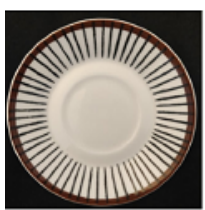

Plate 3

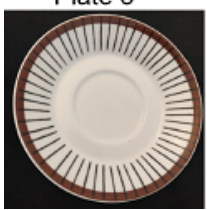

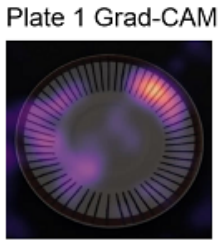

Plate 2 Grad-CAM

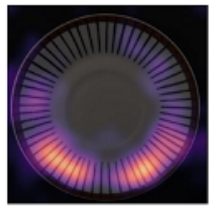

Plate 3 Grad-CAM

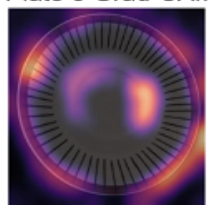

Plate 4

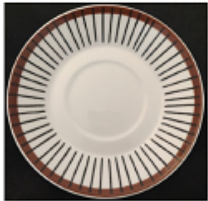

Plate 5

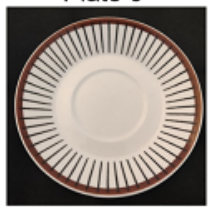

Plate 6

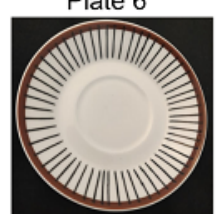

Plate 4 Grad-CAM

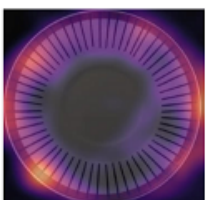

Plate 5 Grad-CAM

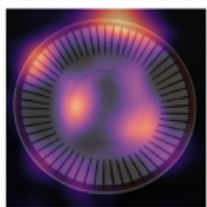

Plate 6 Grad-CAM

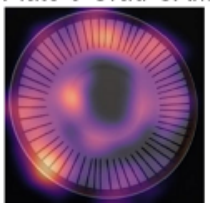

Figure 4: Plate images with and without saliency map (Grad-CAM) overlay. The saliency map lifts out areas important for the decision. All images are identified by the trained neural network with $100 \%$ accuracy.

\subsubsection{Limitations}

We discussed different test setups and chose the one presented above since in that version we do not initially focus or give the participants any possibility to view the saliency maps or reflect over explanations and concepts in general prior to the test. The number of participants $\mathrm{N}=8$ was selected since our primary focus was to verify that human and machine explanations can be compared using scientific explanations.

\subsection{Result and analysis}

In the sections below, we present and analyse the results of our study, outlined in Section 1.3. Phase 1 is identical for all participants. Phase 2 is identical to Phase 1 except that participants 5-8 are introduced to saliency maps. In Phase 3 the result and analysis of the interviews are presented. 
Table 1: Phase 1 test results

\begin{tabular}{|c|c|c|}
\hline \multicolumn{3}{|c|}{ Phase 1 } \\
\hline Participant & Single plate 1 & Multi plate 1 \\
\hline 1 & $83 \%$ & $4 / 6$ \\
2 & $100 \%$ & $6 / 6$ \\
3 & $33 \%$ & $0 / 6$ \\
4 & $22 \%$ & $1 / 6$ \\
5 & $39 \%$ & $4 / 6$ \\
6 & $61 \%$ & $6 / 6$ \\
7 & $56 \%$ & $4 / 6$ \\
8 & $61 \%$ & $6 / 6$ \\
\hline Average correct & $57 \%$ & $65 \%$ \\
\hline
\end{tabular}

\subsubsection{Phase 1}

In this phase, the study participants are introduced to the test setting through a learning phase and two tests. Numeric results from the tests are presented in columns two and three in Table 1. Column two displays the percentage correct identification out of 18 separate plate presentations, column three displays, the number of correctly identified plates when all six plates are visible.

The five minutes time limit allocated for the training was enough since none of the participants used all of it. Participant 2 spent the shortest time for this phase.

In the single-plate part, the participants had to rely on characteristic attributes to identify each plate, without direct visual reference to the other plates. The technique needed for this test relates closer to causal attribution than contrastive attribution. All participants performed better than random (17\%).

The multi-plate part allowed the participants to use contrastive techniques to identify the plates. The participant's results are in line with the results in the single plate test in that the participants that performed well in that test performed well in this test and vice versa. In this small test, the fact that participants could use contrastive attribution to identify plates did not make the result significantly better than in the single plate test. 
Table 2: Phase 2 test results

\begin{tabular}{|c|c|c|}
\hline \multicolumn{3}{|c|}{ Phase 2 } \\
\hline Participant & Single plate 2 & Multi plate 2 \\
\hline 1 & $89 \%$ & $6 / 6$ \\
2 & $100 \%$ & $6 / 6$ \\
3 & $44 \%$ & $2 / 6$ \\
4 & $83 \%$ & $6 / 6$ \\
5 (Saliency map) & $78 \%$ & $6 / 6$ \\
6 (Saliency map) & $78 \%$ & $6 / 6$ \\
7 (Saliency map) & $83 \%$ & $6 / 6$ \\
8 (Saliency map) & $72 \%$ & $4 / 6$ \\
\hline Average correct & $78 \%$ & $88 \%$ \\
\hline
\end{tabular}

\subsubsection{Phase 2}

In this phase, the study participants repeated Phase 1, except the exposure to saliency maps. Numeric results from the tests are presented in Table 2. None of the participants used the entire five minutes, with Participant 2, again, using the shortest time. The participants performed better or on the same level as in Phase 1. Participant 8, in the multi-plate test, was the only exception to this. Exposure to saliency maps for participants 5-8 gave us indications related to their possible usage, but from our small sample, no conclusion related to improved test results can be drawn.

\subsubsection{Phase 3: Interview}

The central part of the study were the interviews we conducted directly after Phase 1 and Phase 2. We opened the discussion by asking the participants to explain their memory strategy. With this question the participants formulated, using characteristic attributes they grouped and named, their strategies to distinguish the plates from each other. The participants focused either on uniqueness (causal attribution) or differences (contrastive attribution), or a combination of these. Two participants lifted out tactile attributes like 'feels flat' and that the plates had different thickness. This then points towards opportunities related to using tactile information currently not present in the data category.

In the content analysis, we searched for categories that could be used to build statements useful in scientific explanations. We refer to these as concepts that in relation to the domain, are meaningful, coherent and 
important (Ghorbani et al., 2019) and at the same time mutually exclusive and exhaustive (Graneheim \& Lundman, 2004). By interpreting meaning units to form concepts and sub-concepts we could, on levels related to Overton's categories, create a taxonomy useful to identify the plates. As an example we grouped adjectives concerning the lines, like blurred, noisy and smeared as the concept 'Line blurred'. We then got the concepts and sub-concepts presented in Table 3. The concepts we selected are then mutually exclusive on one categorical level. For example, a concept categorised as theories related to the domain hand-painted porcelain, like, 'thumbprint' can overlap the more general concept 'blurred line' that we defined as belonging to the kind Spisa Ribb.
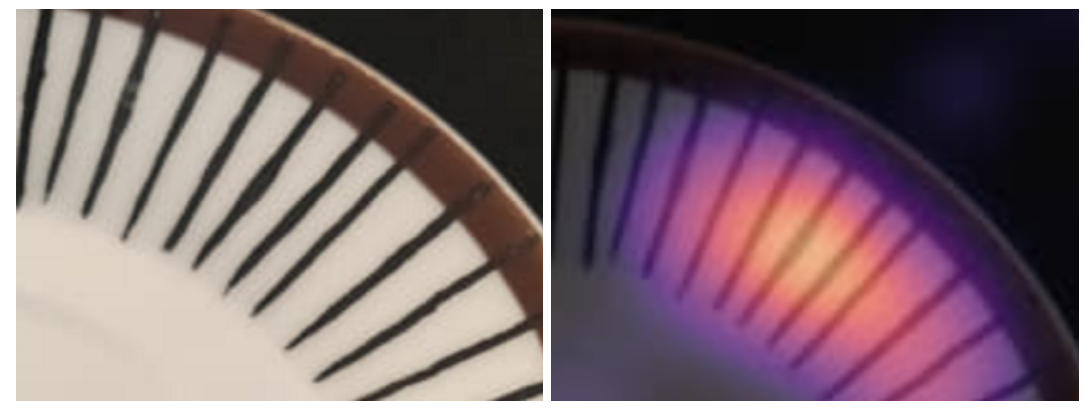

Figure 5: Detail of plate 1 highlighted by the saliency map. The images show a section with the thicker decoration lines that are likely the differing details that the saliency map has lifted out.
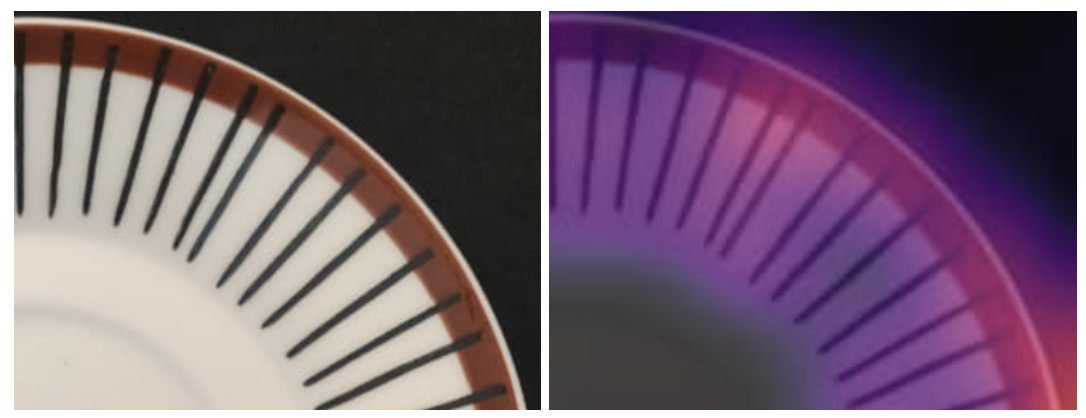

Figure 6: Detail of plate 4 highlighted by the saliency map. The images show a section with plate lines close to each other. These are noticed by the study participants but only to a minor extent highlighted by the saliency map.

As with any taxonomy, the concepts can be discussed, rearranged, composed and decomposed. Once identified and agreed upon, humanunderstandable concepts that relate to Spisa Ribb plates can function as statements useful to build explanations. We identified concepts used in relation to Overton's scientific explanation categories: model, entities and 
kinds.

Most concepts related to the kind category 'Spisa Ribb' created by participants with no special knowledge concerning porcelain. One possible categorisation of these can be seen in Table 3 . It can be noted that concepts related to lines were used 24 times and other concepts 10 times by the participants. The most common concept was 'Line-Line relation' that relates to comparably shorter or longer distances between lines (See Figure 6). This concept was used 10 times. Most of these concepts related to the Spisa Ribb domain are built using adjectives and explanations build to a larger extent on contrasts. For example, plate 2 'Two lines close' or plate 6 'Three lines close to each other with three other lines close to each other on the opposite side.'

Table 3: Concepts related to the kind category Spisa Ribb.

\begin{tabular}{|l|l|}
\hline \multicolumn{2}{|c|}{ Kind category concepts } \\
\hline Line concepts & Other concepts \\
\hline Width & Relation (Not line) \\
Length & Paint stain \\
Blurred & Glaze imperfection \\
Dotted & Symmetry \\
Line-Line relation & Imperfection (Not glaze) \\
Line-Other relation & Tactile \\
\hline
\end{tabular}

Even if we selected plates without concepts connected to the entity category, one user found dirt on one plate that he used to identify this specific plate. Other entity related concepts in timeworn plates could be 'splinter' or 'cracks' that follows one instance of a plate. These concepts belong to and are meaningful for a domain different from the concepts related to the kind.

The participant with a perfect score in the test had domain knowledge related to hand-painted plates and could therefore use other concepts to distinguish the plates. We defined these concepts as belonging to the model category, based on theories related to hand-painted porcelain. Concepts we identified belonging to the domain hand-painted are 'thumbprint', 'does not look hand-painted' and perhaps 'smeared lines'. This participant managed to identify all plates using only one concept for each plate.

There was no consensus between the participants regarding which explanans was used to create the explanation for an identification. This points 
towards a rich and diverse explanandum where no single explanation can be defined as the best. The only distinguishing attribution we identified that was used more than once in an explanation was the Line-Line relation on plate 3 (See Figure 6). This specific characteristic was used by two participants to uniquely identify plate 4 .

None of the participants questioned that saliency maps highlighted causal attributes in the plates correctly, but opinions regarding their usefulness differed. Participant 6 explained that he seemed to use a more general strategy compared to the saliency map, which, in his opinion, can spot very subtle differences in the plates. He also proposed that a text complementing the characteristic attributes would be very beneficial. Another participant (Participant 8) became very interested in studying details presented by saliency maps. While she was doing it, she felt that it was sometimes beneficial and sometimes confusing. When the saliency map highlighted an attribute on a plate, that she had not observed, she felt that it was easy to incorporate the explanation into her own explanations.

Furthermore, the participants noted that when the saliency map explanation highlighted a part of a plate that overlapped with their memory strategy, it boosted their confidence in their strategy.

The saliency maps usefulness for this domain can be questioned since, even if the classification is $100 \%$, and the explanandum is shared. For example in Figure 5 the human concept we named 'Line width' can be identified and is also highlighted in the saliency map. On the other hand, the concept we named 'Line-Line relation', used by two participants, in Figure 6 is only slightly exposed as significant for the decision in the saliency map.

\subsection{Discussion}

In the first section, we present our findings related to the structure of scientific explanations. In the next section, we use scientific explanations as a lens to suggest new design approaches for XAI.

\subsubsection{Structure of ML Explanations}

Our participants identified and named concepts useful to build explanations. This approach, except for naming of concepts, resemble the manner a neural network during training creates internal representations that can be lifted out, as causal attributions, using, for example, a saliency map. Since most study participants lack any deeper knowledge related to hand-painted 
porcelain they could not name concepts related to that domain, instead they used more general concepts, related to kinds, like 'blurred lines', 'narrow lines' or 'paint stains'. The concepts selected by the participant with a perfect score (Participant 2) differed from the other participants in that she knowingly looked for concepts that originated from the theories category and her mental model of the domain hand-painted porcelain ('thumbprint', 'smeared lines', et cetera.) (see Section 1.2) (Overton, 2012; Woodward, 2019).

The core relation we study, both for our study participants and the ML system, are differences between entities. Our focus on this core relation affects the selection of causal attributes the saliency map lifts out. In the study we found an overlap between these attributes and the concepts humans find useful when the focus is the same core relation. If the core relationship for the classification, for example, instead was between kinds and the goal was to classify in two classes; hand-painted and decor-printed porcelain, the human concepts need to emanate from theories related to differences between hand-made and machine-made items. A setting like ours could then be used by a human explainee to discover concepts related to hand-painted versus decor printed plates.

\subsubsection{Explanations from a Human Perspective}

The participant's capability to identify and name concepts indicates an opportunity, for an explainee, to name and group concepts through interaction with a saliency map, or other methods that lifts out causal attributes (Lundberg \& Lee, 2017; Lakkaraju et al., 2019; Ribeiro et al., 2016; Gilpin et al., 2019). In that way, a taxonomy the ML system can use to create causal attributions can be created and expanded towards a semantic level, using concepts identified and named by human users during usage. This borders recent work by Akula et al. (2020) that identifies concepts like, 'stripedness', 'beard', 'horn', et cetera that can be used to create counterfactual explanations building on minimal concept changes that changes the classification, from for example goat to sheep. Our problem differs in that the concepts identified can be very subtle, relative and can even be, in a system that enhances human capabilities, not visible to the naked eye. Our work is then more in line with Ghorbani et al. (2019)'s work on concept-based explanations. That work focus on identifying lowlevel concepts using predefined ImageNet classes while our work is more focused on designing for, during usage, a human, identifying emergent 
concepts in an explanandum shared between humans and an ML system. In all cases, scientific explanations, using the structure Overton proposes can, as shown in the discussion above, add a complementing theoretical base to XAI.

To identify a plate, the participants either looked for uniqueness or contrast. In a simple case like this with a limited number of entities, choosing one cause can identify a specific plate. The participants exposed to the saliency maps tried to match and incorporate these causal attributions with their own concepts and explanations. Relating these attributions to categories in scientific explanations then adds an extra ontological layer. A design approach that allows a user to, in an iterative fashion, select among existing methods that summarise reasons for a decision (Lundberg \& Lee, 2017; Lakkaraju et al., 2019; Ribeiro et al., 2016; Gilpin et al., 2019) and presentation techniques (Lipton, 2016; Barredo Arrieta et al., 2020; Wang et al., 2019) is an interesting line of future research. Causal attributes can then be inspected, viewed and judged by the user and be used to better understand causes for a decision.

This parallels the notion that explanations in a human context take the form of conversations. An iterative process, to build a better understanding for an explainee, driven by the explainee asking why questions in a conversational manner (Hilton, 1990). From a human perspective this points away from ML systems that try to find one explanation that is objectively best and understandable for all users, instead, it opens up for a setting where a user interacts with the, for the ML system, reachable part of the explanandum.

\subsection{Conclusion}

This paper set out to explore how human to human explanations in the form of scientific explanations can be used to better understand limitations in post hoc explanations for ML systems. We illustrate the difference between human and ML explanations by applying the structure and terminology of scientific explanations to a design experiment. Even if our design experiment is specifically focused on lifting out internal knowledge representations in a neural network in the form of causal attributions for entities, scientific explanations have a wider scope. A design approach for XAI that builds on scientific explanations is therefore likely to be generalisable into a setting that makes better use of human-understandable concepts that matches internal representations created during the neural 
networks training phase.

In terms of future research, we particularly suggest inquiring into different forms and types of explanatory methods in an iterative and interactive setting -a setting that can bring a searchable and sortable plethora of causal attributions forward as evidence for a decision to a user with domain knowledge.

\section{References}

Abdul, A., Vermeulen, J., Wang, D., Lim, B. Y., \& Kankanhalli, M. (2018). Trends and trajectories for explainable, accountable and intelligible systems: An HCI research agenda. In Conference on Human Factors in Computing Systems - Proceedings (Vol. 2018-April, pp. 1-18). New York, NY, USA: ACM. doi: 10.1145/3173574.3174156

Adadi, A., \& Berrada, M. (2018). Peeking Inside the Black-Box: A Survey on Explainable Artificial Intelligence (XAI). IEEE Access, 6, 52138-52160. doi: 10.1109/ACCESS.2018.2870052

Akula, A., Wang, S., \& Zhu, S.-C. (2020). CoCoX: Generating Conceptual and Counterfactual Explanations via Fault-Lines. Proceedings of the AAAI Conference on Artificial Intelligence, 34(03), 2594-2601. doi: 10.1609/ aaai.v34i03.5643

Amershi, S., Fogarty, J., Kapoor, A., \& Tan, D. (2009). Overview-based example selection in end-user interactive concept learning. In UIST 2009 - Proceedings of the 22nd Annual ACM Symposium on User Interface Software and Technology (pp. 247-256). doi:10.1145/1622176.1622221

Barredo Arrieta, A., Díaz-Rodríguez, N., Del Ser, J., Bennetot, A., Tabik, S., Barbado, A., ... Herrera, F. (2020). Explainable Artificial Intelligence (XAI): Concepts, taxonomies, opportunities and challenges toward responsible AI. Information Fusion, 58, 82-115. doi: 10.1016/j.inffus .2019 .12 .012

Bengio, Y., Courville, A., \& Vincent, P. (2013). Representation learning: A review and new perspectives. IEEE Transactions on Pattern Analysis and Machine Intelligence, 35(8), 1798-1828. doi: 10.1109/TPAMI.2013.50

Biran, O., \& Cotton, C. (2017). Explanation and Justification in Machine Learning: A Survey. In IJCAI Workshop on Explainable AI (XAI) (Vol. 8, pp. 8-14).

Chollet, F. (2019). On the Measure of Intelligence. arXiv preprint arXiv:1911.01547, 64.

Doshi-Velez, F., \& Kim, B. (2017). Towards a Rigorous Science of Interpretable Machine Learning. arXiv preprint arXiv:1702.08608.

Ferreira, A. C., Silva, L. R., Renna, F., Brandl, H. B., Renoult, J. P., Farine, D. R., ... Doutrelant, C. (2020). Deep learning-based methods for individual recognition in small birds. Methods in Ecology and Evolution, 11(9), 1072-1085. doi: 10.1111/2041-210X.13436

Genone, J., \& Lombrozo, T. (2012). Concept possession, experimental 
semantics, and hybrid theories of reference. Philosophical Psychology, 25(5), 717-742. doi: 10.1080/09515089.2011.627538

Gentner, D., \& Markman, A. B. (1997). Structure Mapping in Analogy and Similarity. American Psychologist, 52(1), 45-56. doi: 10.1037/ 0003-066X.52.1.45

Ghorbani, A., Wexler, J., Zou, J., \& Kim, B. (2019). Towards Automatic Concept-based Explanations. arXiv preprint arXiv 1902.03129.

Gilpin, L. H., Bau, D., Yuan, B. Z., Bajwa, A., Specter, M., \& Kagal, L. (2019). Explaining explanations: An overview of interpretability of machine learning. In Proceedings - 2018 IEEE 5th International Conference on Data Science and Advanced Analytics, DSAA 2018 (pp. 80-89). IEEE. doi: 10.1109/DSAA.2018.00018

Gonzalez-Garcia, A., Modolo, D., \& Ferrari, V. (2018, 5). Do Semantic Parts Emerge in Convolutional Neural Networks? International Journal of Computer Vision, 126(5), 476-494. doi: 10.1007/s11263-017-1048-0

Graneheim, U., \& Lundman, B. (2004). Qualitative content analysis in nursing research: concepts, procedures and measures to achieve trustworthiness. Nurse Education Today, 24(2), 105-112. doi: 10.1016/J.NEDT.2003.10 .001

Guidotti, R., Monreale, A., Ruggieri, S., Turini, F., Giannotti, F., \& Pedreschi, D. (2018). A survey of methods for explaining black box models. ACM Computing Surveys, 51(5), 42. doi: 10.1145/3236009

Gunning, D. (2017). Explainable artificial intelligence (xai). Defense Advanced Research Projects Agency (DARPA), nd Web, 2, 2.

Hempel, C. G., \& Oppenheim, P. (1948). Studies in the Logic of Explanation. Philosophy of Science, 15(2), 135-175. doi: 10.1086/286983

Hilton, D. J. (1990). Conversational processes and causal explanation. Psychological Bulletin, 107(1), 65-81. doi: 10.1037/0033-2909.107.1 .65

HLEG. (2019). Ethics Guidelines for Trustworthy AI. European Commission, 32(May), 1-39.

Hoffman, R. R., Clancey, W. J., \& Mueller, S. T. (2020). Explaining AI as an exploratory process: The peircean abduction model. arXiv preprint arXiv 2009.14795.

Kim, B., Wattenberg, M., Gilmer, J., Cai, C., Wexler, J., Viegas, F., \& Sayres, R. (2018). Interpretability beyond feature attribution: Quantitative Testing with Concept Activation Vectors (TCAV). In 35th International Conference on Machine Learning, ICML 2018.

Ko, A. J., LaToza, T. D., \& Burnett, M. M. (2013). A practical guide to controlled experiments of software engineering tools with human participants. Empirical Software Engineering, 20(1), 110-141. doi: 10 .1007/s10664-013-9279-3

Koh, P. W., Nguyen, T., Tang, Y. S., Mussmann, S., Pierson, E., Kim, B., \& Liang, P. (2020). Concept Bottleneck Models. In International Conference on Machine Learning. 
Kornblith, S., Norouzi, M., Lee, H., \& Hinton, G. (2019). Similarity of neural network representations revisited. In 36th International Conference on Machine Learning, ICML 2019 (Vol. 2019-June, pp. 6156-6175).

Kulesza, T., Burnett, M., Wong, W. K., \& Stumpf, S. (2015). Principles of Explanatory Debugging to personalize interactive machine learning. In Proceedings of the 20th International Conference on Intelligent User Interfaces (Vol. 2015-Janua, pp. 126-137). Association for Computing Machinery. doi: 10.1145/2678025.2701399

Lakkaraju, H., Kamar, E., Caruana, R., \& Leskovec, J. (2019). Faithful and Customizable Explanations of Black Box Models. In Proceedings of the 2019 AAAI/ACM Conference on AI, Ethics, and Society (pp. 131-138) (pp. 131-138). Honolulu: ACM.

Lecun, Y., Bengio, Y., \& Hinton, G. (2015). Deep learning. Nature, 521(7553), 436-444. doi: 10.1038/nature14539

Lindvall, M., Molin, J., \& Löwgren, J. (2018). From machine learning to machine teaching. Interactions, 25(6), 52-57. doi: 10.1145/3282860

Lipton, P. (1990, 3). Contrastive Explanation. Royal Institute of Philosophy Supplement, 27, 247-266. doi: 10.1017/s1358246100005130

Lipton, Z. C. (2016). The Mythos of Model Interpretability. Communications of the ACM, 61(10), 35-43. doi: 10.1145/3233231

Lu, J., Lee, D. D., Kim, T. W., \& Danks, D. (2019). Good Explanation for Algorithmic Transparency. SSRN Electronic Journal. doi: 10.2139/ ssrn. 3503603

Lundberg, S. M., \& Lee, S. I. (2017). A unified approach to interpreting model predictions. In Advances in Neural Information Processing Systems (pp. 4766-4775). MIT press.

Marcus, G. (2018). Deep Learning: A Critical Appraisal. arXiv preprint arXiv 1801.00631, 27.

Medin, D. L., Goldstone, R. L., \& Gentner, D. (1993). Respects for Similarity. Psychological Review, 100(2), 254-278. doi: 10.1037/0033-295X.100.2 .254

Miller, T. (2019). Explanation in artificial intelligence: Insights from the social sciences. Artificial Intelligence, 267, 1-38. doi: 10.1016/j.artint.2018 .07 .007

Murphy, G. (2004). The Big Book of Concepts. MIT press. doi: 10.7551/ mitpress/1602.001.0001

Myers, M. D., \& Newman, M. (2007). The qualitative interview in IS research: Examining the craft. Information and Organization, 17(1), 2-26. doi: 10.1016/j.infoandorg.2006.11.001

Overton, J. A. (2012). Explanation in Science (Doctoral dissertation, University of Western Ontario).

Overton, J. A. (2013). "Explain" in scientific discourse. Synthese, 190, 1383-1405. doi: 10.1007/s11229-012-0109-8

Ribeiro, M. T., Singh, S., \& Guestrin, C. (2016). "Why should i trust you?" Explaining the predictions of any classifier. In Proceedings of the 
22nd ACM SIGKDD International Conference on Knowledge Discovery and Data Mining (Vol. 13-17, pp. 1135-1144). ACM. doi: 10.1145/ 2939672.2939778

Ribera, M., \& Lapedriza, A. (2019). Can we do better explanations? A proposal of user-centered explainable AI. In IUI Workshops (Vol. 2327, p. 38).

Samek, W., Montavon, G., Lapuschkin, S., Anders, C. J., \& Müller, K. R. (2020). Toward interpretable machine learning: Transparent deep neural networks and beyond.

Selvaraju, R. R., Cogswell, M., Das, A., Vedantam, R., Parikh, D., \& Batra, D. (2020). Grad-CAM: Visual Explanations from Deep Networks via Gradient-Based Localization. International Journal of Computer Vision, 128(2), 336-359. doi: 10.1007/s11263-019-01228-7

Wang, D., Yang, Q., Abdul, A., \& Lim, B. Y. (2019). Designing theorydriven user-centric explainable AI. In Conference on Human Factors in Computing Systems - Proceedings. Association for Computing Machinery. doi: 10.1145/3290605.3300831

Woodward, J. (2019). Scientific Explanation. In The Stanford Encyclopedia of Philosophy. 

ISBN 978-91-7877-186-8 (print)

ISBN 78-91-7877-187-5 (pdf)

MALMÖ UNIVERSITY 20506 MALMÖ, SWEDEN WWW.MAU.SE 